TRANSACTIONS OF THE

AMERICAN MATHEMATICAL SOCIETY

Volume 362, Number 6, June 2010, Pages 3319-3363

S 0002-9947(09)05048-X

Article electronically published on December 22, 2009

\title{
CHARACTERIZATIONS OF LOJASIEWICZ INEQUALITIES: SUBGRADIENT FLOWS, TALWEG, CONVEXITY
}

\author{
JÉRÔME BOLTE, ARIS DANIILIDIS, OLIVIER LEY, AND LAURENT MAZET
}

\begin{abstract}
The classical Łojasiewicz inequality and its extensions for partial differential equation problems (Simon) and to o-minimal structures (Kurdyka) have a considerable impact on the analysis of gradient-like methods and related problems: minimization methods, complexity theory, asymptotic analysis of dissipative partial differential equations, and tame geometry. This paper provides alternative characterizations of this type of inequality for nonsmooth lower semicontinuous functions defined on a metric or a real Hilbert space. In the framework of metric spaces, we show that a generalized form of the Łojasiewicz inequality (hereby called the Kurdyka-Łojasiewicz inequality) is related to metric regularity and to the Lipschitz continuity of the sublevel mapping, yielding applications to discrete methods (strong convergence of the proximal algorithm). In a Hilbert setting we further establish that asymptotic properties of the semiflow generated by $-\partial f$ are strongly linked to this inequality. This is done by introducing the notion of a piecewise subgradient curve: such curves have uniformly bounded lengths if and only if the KurdykaŁojasiewicz inequality is satisfied. Further characterizations in terms of talweg lines - a concept linked to the location of the less steepest points at the level sets of $f$ - and integrability conditions are given. In the convex case these results are significantly reinforced, allowing us in particular to establish a kind of asymptotic equivalence for discrete gradient methods and continuous gradient curves. On the other hand, a counterexample of a convex $C^{2}$ function in $\mathbb{R}^{2}$ is constructed to illustrate the fact that, contrary to our intuition, and unless a specific growth condition is satisfied, convex functions may fail to fulfill the Kurdyka-Łojasiewicz inequality.
\end{abstract}

\section{Contents}

1. Introduction

2. KŁ-inequality is a metric regularity condition 3324

2.1. Metric regularity and global error bounds 3324

2.2. Metric regularity and KE-inequality 3327

3. KE-inequality in Hilbert spaces 3328

3.1. Elements of nonsmooth analysis $\quad 3329$

3.2. Subgradient curves: Basic properties 3330

3.3. Characterizations of the KE-inequality 3332

3.4. Application: Convergence of the proximal algorithm 3338

Received by the editors February 7, 2008 and, in revised form, March 11, 2009

2010 Mathematics Subject Classification. Primary 26D10; Secondary 03C64, 37N40, 49J52, $65 \mathrm{~K} 10$.

Key words and phrases. Łojasiewicz inequality, gradient inequalities, metric regularity, subgradient curve, talweg, gradient method, convex functions, global convergence, proximal method. 
4. Convexity and KE-inequality 3339

4.1. Lengths of subgradient curves for convex functions 3340

4.2. KL-inequality for convex functions 3341

4.3. A smooth convex counterexample to the KE-inequality 3344

4.4. Asymptotic equivalence for discrete and continuous dynamics 3353

5. Annex 3356

5.1. Technical results 3356

5.2. Explicit gradient method 3360

Acknowledgement $\quad 3361$

References 3361

\section{INTRODUCTION}

The Łojasiewicz inequality is a powerful tool to analyze convergence of gradientlike methods and related problems. Roughly speaking, this inequality is satisfied by a $C^{1}$ function $f$ if for some $\rho \in\left[\frac{1}{2}, 1\right)$ the quantity

$$
|f-f(\bar{x})|^{\rho}\|\nabla f\|^{-1}
$$

remains bounded around any (possibly critical) point $\bar{x}$. This result is named after S. Łojasiewicz (see [42, 43]), who was the first to establish its validity for the class of real-analytic functions. We refer to [38] for an extension of this result to the class of $C^{1}$ subanalytic functions and to [8] for a further extension to (nonsmooth) lower semicontinuous subanalytic functions. At the same time it is known that the Łojasiewicz inequality fails for $C^{\infty}$ functions in general (see the classical example of the function $x \longmapsto \exp \left(-1 / x^{2}\right)$ if $x \neq 0$, and 0 if $x=0$ around the point $\left.\bar{x}=0\right)$.

A generalized form of this inequality has been introduced by Kurdyka in [37. In the framework of a $C^{1}$ function $f$ defined on a real Hilbert space $[H,\langle\cdot, \cdot\rangle]$, and assuming for simplicity that $\bar{f}=0$ is a critical value, this generalized inequality (that we hereby call the Kurdyka-Lojasiewicz inequality, or in short, the KE-inequality) states that

$$
\|\nabla(\varphi \circ f)(x)\| \geq 1
$$

for some continuous function $\varphi:[0, r) \rightarrow \mathbb{R}, C^{1}$ on $(0, r)$ with $\varphi^{\prime}>0$ and all $x$ in $[0<f<r]:=\{y \in H: 0<f(y)<r\}$. The class of such functions $\varphi$ will be further denoted by $\mathcal{K}(0, \bar{r})$; see (8) . Note that the Łojasiewicz inequality corresponds to the case $\varphi(t)=t^{1-\rho}$.

In finite-dimensional spaces it has been shown in 37 that (10) is satisfied by a much larger class of functions, namely, by those that are definable in an o-minimal structure [17, or even more generally by functions belonging to analytic-geometric categories [25. In the meantime the original Łojasiewicz result was used to derive new results in the asymptotic analysis of nonlinear heat equations [51, 35] and damped wave equations [30. Many results related to partial differential equations followed; see the monograph of Huang [31] for an insight. Other fields of application of (11) are nonconvex optimization and nonsmooth analysis. This was one of the motivations for the nonsmooth $\mathrm{KL}$-inequalities developed in [8, 9]. Due to its considerable impact on several fields of applied mathematics: minimization and algorithms [1, 5, 8, 39, asymptotic theory of differential inclusions [48, neural networks [28, complexity theory [47] (see [47, Definition 3], where functions satisfying 
a KŁ-type inequality are called gradient dominated functions), and partial differential equations [51, 35, 30, 31, we hereby tackle the problem of characterizing such inequalities in a nonsmooth infinite-dimensional setting and provide further clarifications for several application aspects. Our framework is rather broad (infinite dimensions, nonsmooth functions); nevertheless, to the best of our knowledge, most of the present results are also new in a smooth finite-dimensional framework: readers who feel unfamiliar with notions of nonsmooth and variational analysis may, as a first stage, consider that all functions involved are differentiable, replace subdifferentials by usual derivatives and subgradient systems by smooth ones.

A first part of this work (Section 2) is devoted to the analysis of metric versions of the KE-inequality. The underlying space $H$ is only assumed to be a complete metric space (without any linear structure), the function $f: H \rightarrow \mathbb{R} \cup\{+\infty\}$ is lower semicontinuous and possibly real-extended valued and the notion of a gradient is replaced by the variational notion of a strong-slope [22, 6]. Indeed, introducing the multivalued mapping $F(x)=[f(x),+\infty$ ) (whose graph is the epigraph of $f$ ), the KŁ-inequality (11) appears to be equivalent to the metric regularity of $F: H \rightrightarrows \mathbb{R}$ on an adequate set, where $\mathbb{R}$ is endowed with the metric $d_{\varphi}(r, s)=|\varphi(r)-\varphi(s)|$. This fact is strongly connected to famous classical results in this area (see [23, 45, 32, 49] for example) and in particular to the notion of $\rho$-metric regularity introduced in 32 by Ioffe. Using results on global error-bounds of Ioffe [32, 33, 34, (see also Azé-Corvellec [6]) we show that some global forms of the KE-inequality, as well as metric regularity, are both equivalent to the "Lipschitz continuity" of the sublevel mapping

$$
\left\{\begin{array}{l}
\mathbb{R} \rightrightarrows H \\
r \quad \mapsto[f \leq r]:=\{x \in H: f(x) \leq r\}
\end{array}\right.
$$

where $(0, r) \subset(0,+\infty)$ is endowed with $d_{\varphi}$ and the collection of subsets of $H$ with the "Hausdorff distance". As is shown in a section devoted to applications (Section 3.4), this reformulation is particularly adapted for the analysis of proximal methods involving nonconvex criteria: these results are in the line of [15, [5].

In the second part of this work (Section 3 ), $H$ is a real Hilbert space and $f$ is assumed to be a semiconvex function; i.e., $f$ is the difference of a proper lower semicontinuous convex function and a function proportional to the canonical quadratic form. Although this assumption is not particularly restrictive, it does not aim at full generality. Semiconvexity is used here to provide a convenient framework in which the formulation and the study of subdifferential evolution equations are simple and elegant (2, 13, 21). Using the Fréchet subdifferential (see Definition 8), the corresponding subgradient dynamical system indeed reads

$$
\left\{\begin{array}{l}
\dot{x}(t)+\partial f(x(t)) \ni 0, \text { a.e. on }(0,+\infty), \\
x(0) \in \operatorname{dom} f
\end{array}\right.
$$

where $x(\cdot)$ is an absolutely continuous curve called a subgradient curve. Relying on several works [21, 44, 11, if $f$ is semiconvex, such curves exist and are unique. The asymptotic properties of the semiflow associated to this evolution equation are strongly connected to the KE-inequality. This can be made precise by introducing the following notion: for $T \in(0,+\infty]$, a piecewise absolutely continuous curve $\gamma:[0, T) \rightarrow H$ (with countable pieces) is called a piecewise subgradient curve if $\gamma$ is a solution to (2) where in addition $t \mapsto(f \circ \gamma)(t)$ is nonincreasing (see 
Definition 15 for details). Consider all piecewise subgradient curves lying in a "KLneighborhood", e.g. a slice of level sets. Under a compactness assumption and a condition of Sard type (automatically satisfied in finite dimensions if $f$ belongs to an o-minimal class), their lengths are uniformly bounded if and only if $f$ satisfies the KŁ-inequality in its nonsmooth form (see [9]); that is, for all $x \in[0<f<r]$,

$$
\|\partial(\varphi \circ f)(x)\|_{-}:=\inf \{\|p\|: p \in \partial(\varphi \circ f)\} \geq 1,
$$

where $\varphi:(0, r) \rightarrow \mathbb{R}$ is a $C^{1}$ function bounded from below such that $\varphi^{\prime}>0$ (see (8)). A byproduct of this result (though not an equivalent statement, as we show in Section 4.3; see Remark 37(c)) is the fact that bounded subgradient curves have finite lengths and hence converge to a generalized critical point.

Further characterizations are given involving several aspects among which, an integrability condition in terms of the inverse function of the minimal subgradient norm associated to each level set $[f=r]$ of $f$, as well as connections to the following talweg selection problem: given $R>1$, find a piecewise absolutely continuous curve $\theta:(0, r) \rightarrow H$ with finite length such that

$$
\theta(r) \in\left\{x \in[f=r]:\|\partial(\varphi \circ f)(x)\|_{-} \leq R \inf _{y \in[f=r]}\|\partial(\varphi \circ f)(y)\|_{-}\right\} .
$$

The curve $\theta$ is called a talweg. Early connections between the KŁ-inequality and this old concept can be found in [19], 37, and even more clearly in the Ph.D. Thesis of D'Acunto [18. Indeed, under mild assumptions, the existence of such a selection curve $\theta$ characterizes the KL-inequality. The proof relies strongly on the property of the semiflow associated to $-\partial f$. Recent developments of the metric theory of a "gradient" curve ([3]) open the way to a more general approach of these characterizations, and hopefully to new applications in the line of 3, 22.

The analysis of the convex case (that is, $f$ is a convex function) in Section 4, reveals interesting phenomena. In this case, the KE-inequality, whenever true on a slice of level sets, will be true on the whole space $H$ (globalization) and, in addition, the involved function $\varphi$ can be taken to be concave (Theorem 29). This is always the case if a specific growth assumption near the set of minimizers of $f$ is assumed. On the other hand, arbitrary convex functions do not satisfy the KŁ-inequality: this is a straightforward consequence of a classical counterexample, due to Baillon [7, of the existence of a convex function $f$ in a Hilbert space, having a subgradient curve which is not strongly converging to $0 \in \arg \min f$. However, surprisingly, even smooth finite-dimensional coercive convex functions may fail to satisfy the KŁ-inequality, and this even in the case that the lengths of their gradient curves are uniformly bounded. Indeed, using the above-mentioned characterizations and results from [36 and 52, we construct a counterexample of a $C^{2}$ convex function whose set of minimizers is compact and has a nonempty interior (Section 4.3).

As another application we consider abstract explicit gradient schemes for convex functions with a Lipschitz continuous gradient. A common belief is that the analysis of gradient curves and their explicit discretization used in numerical optimization are somehow disconnected problems. We hereby show that this is not always the case by establishing that the piecewise gradient iterations are uniformly bounded if and only if the piecewise subgradient curves are so (cf. Theorem 39). This aspect sheds further light on the (theoretical) stability of convex gradient-like methods (see also [50, Chapter 8]) and the interest of exploring the connections of the KEinequality with the asymptotic study of subgradient-type methods. 
Notation (Multivalued mappings). Let $X, Y$ be two metric spaces and $F: X \rightrightarrows Y$ be a multivalued mapping from $X$ to $Y$, that is, a function from $X$ to $2^{Y}$, associating to each point of $X$ a subset of $Y$. We denote by

$$
\text { Graph } F:=\{(x, y) \in X \times Y: y \in F(x)\}
$$

the graph of the multivalued mapping $F$ (subset of $X \times Y$ ) and by

$$
\operatorname{dom} F:=\{x \in X: \exists y \in Y,(x, y) \in \operatorname{Graph} F\}
$$

its domain (subset of $X$ ).

(Single-valued functions) Given a function $f: X \longrightarrow \mathbb{R} \cup\{+\infty\}$ we define its epigraph by

$$
\text { epi } f:=\{(x, \beta) \in X \times \mathbb{R}: f(x) \leq \beta\} .
$$

We say that the function $f$ is proper (respectively, lower semicontinuous) if the above set is nonempty (respectively, closed). Let us recall that the domain of the function $f$ is defined by

$$
\operatorname{dom} f:=\{x \in X: f(x)<+\infty\} .
$$

(Level sets) Given $r_{1} \leq r_{2}$ in $[-\infty,+\infty]$ we set

$$
\left[r_{1} \leq f \leq r_{2}\right]:=\left\{x \in X: r_{1} \leq f(x) \leq r_{2}\right\} .
$$

When $r_{1}=r_{2}$ (respectively $r_{1}=-\infty$ ), the above set will be simply denoted by $\left[f=r_{1}\right]$ (respectively $\left[f \leq r_{2}\right]$ ).

(Strong slope) Let us recall from [22] (see also [32, 6]) the notion of a strong slope defined for every $x \in \operatorname{dom} f$ as follows:

$$
|\nabla f|(x)=\limsup _{y \rightarrow x} \frac{(f(x)-f(y))^{+}}{d(x, y)},
$$

where for every $a \in \mathbb{R}$ we set $a^{+}=\max \{a, 0\}$ and we use the convention $0 / 0=0$.

If $[X,\|\cdot\|]$ is a Banach space with (topological) dual space $\left[X^{*},\|\cdot\|_{*}\right]$ and $f$ is a $C^{1}$ finite-valued function, then

$$
|\nabla f|(x)=\|D f(x)\|_{*},
$$

for all $x$ in $X$, where $D f(\cdot)$ is the differential map of $f$. by

(Hausdorff distance) We define the distance of a point $x \in X$ to a subset $S$ of $X$

$$
\operatorname{dist}(x, S):=\inf _{y \in S} d(x, y),
$$

where $d$ denotes the distance on $X$. The Hausdorff distance Dist $\left(S_{1}, S_{2}\right)$ of two subsets $S_{1}$ and $S_{2}$ of $X$ is given by

$$
\operatorname{Dist}\left(S_{1}, S_{2}\right):=\max \left\{\sup _{x \in S_{1}} \operatorname{dist}\left(x, S_{2}\right), \sup _{x \in S_{2}} \operatorname{dist}\left(x, S_{1}\right)\right\} .
$$

Let us denote by $\mathcal{P}(X)$ the collection of all subsets of $X$. In general Dist $(\cdot, \cdot)$ can take infinite values and does not define a distance on $\mathcal{P}(X)$. However if $K(X)$ denotes the collection of nonempty compact subsets of $X$, then $\operatorname{Dist}(\cdot, \cdot)$ defines a proper notion of distance on $K(X)$. In the sequel we deal with multivalued 
mappings $F: X \rightrightarrows Y$ enjoying the following property:

$$
\text { Dist }(F(x), F(y)) \leq k d(x, y),
$$

where $k$ is a positive constant. For simplicity such functions are called Lipschitz continuous, although $[\mathcal{P}(Y)$, Dist $]$ is not a metric space in general.

(Desingularization functions) Given $\bar{r} \in(0,+\infty]$, we set

(8) $\mathcal{K}(0, \bar{r}):=\left\{\varphi \in C([0, \bar{r})) \cap C^{1}(0, \bar{r}): \varphi(0)=0\right.$, and $\left.\varphi^{\prime}(r)>0, \forall r \in(0, \bar{r})\right\}$,

where $C([0, \bar{r}])$ (respectively, $\left.C^{1}(0, \bar{r})\right)$ denotes the set of continuous functions on $[0, \bar{r}]$ (respectively, $C^{1}$ functions on $(0, \bar{r})$ ). The introduction of this class, as well as the employed terminology (desingularization) are justified by (11). Indeed, composing a function $f$, which typically has 0 as a critical value, with an appropriate element $\varphi$ of $\mathcal{K}(0, \bar{r})$ consists in relabeling its level sets in order to "straighten up all derivatives" of $f$ in $[0<f<\bar{r}]$.

Finally throughout this work, $B(x, r)$ denotes the open ball of center $x$ and radius $r>0$ and $\bar{B}(x, r)$ denotes its closure. If $H$ is a Hilbert space, its inner product will be denoted by $\langle\cdot, \cdot\rangle$ and the corresponding norm by $\|\cdot\|$.

\section{KE-INEQUALITY IS A METRIC REGULARITY CONDITION}

Let $X, Y$ be two complete metric spaces, $F: X \rightrightarrows Y$ a multivalued mapping and $(\bar{x}, \bar{y}) \in$ Graph $F$. Let us recall from [32, Definition 1 (loc)] the following definition.

Definition 1 (Metric regularity of multifunctions). Let $k \in[0,+\infty)$. (i) The multivalued mapping $F$ is called $k$-metrically regular at $(\bar{x}, \bar{y}) \in$ Graph $F$ if there exist $\varepsilon, \delta>0$ such that for all $(x, y) \in B(\bar{x}, \varepsilon) \times B(\bar{y}, \delta)$ we have

$$
\operatorname{dist}\left(x, F^{-1}(y)\right) \leq k \operatorname{dist}(y, F(x)) \text {. }
$$

(ii) Let $V$ be a nonempty subset of $X \times Y$. The multivalued mapping $F$ is called $k$-metrically regular on $V$ if $F$ is metrically regular at $(\bar{x}, \bar{y})$ for every $(\bar{x}, \bar{y}) \in$ Graph $F \cap V$.

We refer to Ioffe [32, 33, Mordukhovich 45, Penot 49], Dontchev-LewisRockafellar [23, Dontchev-Quincampoix-Zlateva 24] and the references therein for historical comments, examples, other characterizations and applications of the notion of metric regularity.

2.1. Metric regularity and global error bounds. The following result reveals that the Kurdyka-Lojasiewicz inequality and metric regularity are equivalent concepts (see Corollary 4 and Remark [5). The equivalence $[(\mathrm{ii}) \Leftrightarrow($ iii)] can be found in 6. Theorem 2.1] and can also be deduced by previous works of Ioffe (see 32, Theorem 2b], 33 and 34). Recently J.-N. Corvellec informed us that Theorem 2 below can be obtained using an alternative much simpler argument, avoiding in particular the implication (i) $\Rightarrow$ (ii) and the use of Zorn's lemma. However, a direct proof of this implication as well as a similar argument used in the construction of the talweg ( $c f$. Definition [17) in Theorem 18 (ii) $\Rightarrow$ (iv) seem to require the Zorn lemma.

Theorem 2. Let $X$ be a complete metric space, $f: X \longrightarrow \mathbb{R} \cup\{+\infty\}$ a lower semicontinuous function and $r_{0}>0$. Assume that $\left[0<f<r_{0}\right] \neq \emptyset$. The following assertions are equivalent and imply the nonemptiness of the sublevel set $[f \leq 0]$. 
(i) The multivalued mapping

$$
F:\left\{\begin{array}{rll}
X & \rightrightarrows & \mathbb{R} \\
x & \longmapsto & {[f(x),+\infty)}
\end{array}\right.
$$

is $k$-metrically regular on $\left[0<f<r_{0}\right] \times\left(0, r_{0}\right)$;

(ii) for all $r \in\left(0, r_{0}\right)$ and $x \in\left[0<f<r_{0}\right]$,

$$
\operatorname{dist}(x,[f \leq r]) \leq k(f(x)-r)^{+} ;
$$

(iii) for all $x \in\left[0<f<r_{0}\right]$,

$$
|\nabla f|(x) \geq \frac{1}{k}
$$

Proof. According to [16, Theorem 2.2] the sublevel set $[f \leq 0]$ is nonempty whenever (iii) holds. For the equivalence of (ii) and (iii), see [6. Theorem 2.1]. We give below a direct proof of the equivalence $[(\mathrm{i}) \Leftrightarrow(\mathrm{ii})]$. Definition 1 (metric regularity of multifunctions) yields the following restatement for (i):

$(i)_{1}$ For every $(\bar{x}, \bar{r}) \in$ Graph $F$ with $\bar{x} \in\left[0<f<r_{0}\right]$ and $\bar{r} \in\left(0, r_{0}\right)$, there exist $\varepsilon, \delta>0$ such that

$$
\left.\begin{array}{l}
x \in B(\bar{x}, \varepsilon) \cap\left[0<f<r_{0}\right] \\
r \in\left[(\bar{r}-\delta, \bar{r}+\delta) \cap\left(0, r_{0}\right)\right]
\end{array}\right\} \Longrightarrow \operatorname{dist}(x,[f \leq r]) \leq k(f(x)-r)^{+} .
$$

Clearly $(i) \Rightarrow(i)_{1}$. Now, in order to prove $(i)_{1} \Rightarrow(i)$, consider $(\bar{x}, \bar{r}) \in \operatorname{Graph} F \cap$ $\left[0<f<r_{0}\right] \times\left(0, r_{0}\right)$. Take $\varepsilon$ and $\delta$ positive given by $(i)_{1}$ such that $0<\bar{r}-\delta<$ $\bar{r}+2 \delta<r_{0}, \varepsilon \leq k\left(r_{0}-\bar{r}-2 \delta\right)$ and $f$ is positive on $B(\bar{x}, \varepsilon)$ ( $f$ is lower semicontinuous, so $[f>0]$ is open). For any $(x, r) \in B(\bar{x}, \varepsilon) \times(\bar{r}-\delta, \bar{r}+\delta)$, we have $r \in\left(0, r_{0}\right)$ and $f(x)>0$. Thus if $f(x)<r_{0}$, by $(i)_{1}$ we have

$$
\operatorname{dist}(x,[f \leq r]) \leq k(f(x)-r)^{+}=k \operatorname{dist}(r, F(x)) .
$$

If $f(x) \geq r_{0}$, then

$$
\begin{aligned}
\operatorname{dist}(x,[f \leq r]) & \leq \operatorname{dist}(x, \bar{x})+\operatorname{dist}(\bar{x},[f \leq r]) \leq \varepsilon+k(f(\bar{x})-r)^{+} \leq \varepsilon+k \delta \\
& \leq k\left(r_{0}-\bar{r}-\delta\right) \leq k\left(r_{0}-r\right) \leq k(f(x)-r)^{+}=k \operatorname{dist}(r, F(x)) .
\end{aligned}
$$

Thus $(i)_{1} \Rightarrow(i)$.

It is now straightforward to see that $(i i) \Longrightarrow(i)$; thus it remains to prove that $(i)_{1} \Longrightarrow(i i)$. To this end, fix any $k^{\prime}>k, r_{1} \in\left(0, r_{0}\right)$ and $x_{1} \in\left[f=r_{1}\right]$. We shall prove that

$$
\operatorname{dist}\left(x_{1},[f \leq s]\right) \leq k^{\prime}\left(r_{1}-s\right), \quad \text { for all } s \in\left(0, r_{1}\right] .
$$

Claim 1. Let $r \in\left(0, r_{0}\right)$ and $x \in[f=r]$. Then there exist $r^{-}<r$ and $x^{-} \in\left[f=r^{-}\right]$ such that

$$
d\left(x, x^{-}\right) \leq k^{\prime}\left(r-r^{-}\right)
$$

with dist $(x,[f \leq s]) \leq k^{\prime}(r-s)$, for all $s \in\left[r^{-}, r\right]$.

Proof of Claim 1. Apply $(i)_{1}$ at $(x, r) \in$ Graph $F$ to obtain the existence of $\rho \in$ $(0, r)$ such that dist $(x,[f \leq s]) \leq k(r-s)$ for all $s \in[\rho, r]$. Since $k^{\prime}>k$ there exists $x^{-} \in[f \leq \rho]$ satisfying

$$
d\left(x, x^{-}\right)<\frac{k^{\prime}}{k} \operatorname{dist}(x,[f \leq \rho]),
$$


which in view of (11) yields

$$
d\left(x, x^{-}\right)<k^{\prime}(r-\rho) .
$$

To conclude, set $r^{-}=f\left(x^{-}\right) \leq \rho$ and observe that for any $s \in\left[r^{-}, \rho\right]$ we have

$$
\operatorname{dist}(x,[f \leq s]) \leq d\left(x, x^{-}\right) \leq k^{\prime}(r-\rho) \leq k^{\prime}(r-s)=k^{\prime}(f(x)-s) .
$$

This completes the proof of the claim.

Let $\mathcal{A}$ be the set of all families $\left\{\left(x_{i}, r_{i}\right)\right\}_{i \in I} \subset\left[f \leq r_{1}\right] \times \mathbb{R}$ containing $\left(x_{1}, r_{1}\right)$ such that

$-\left(\mathrm{P}_{1}\right) \quad f\left(x_{i}\right)=r_{i}$ for all $i \in I$ and $r_{i} \neq r_{j}$, for $i \neq j$;

- $\left(\mathrm{P}_{2}\right) \quad$ if $i, j \in I$ and $r_{i}<r_{j}$, then $d\left(x_{j}, x_{i}\right) \leq k^{\prime}\left(r_{j}-r_{i}\right)$;

$-\left(\mathrm{P}_{3}\right) \quad$ for $r^{*}=\inf \left\{r_{i}: i \in I\right\}$ and for $s \in\left(r^{*}, r_{1}\right]$ we have:

$$
\operatorname{dist}\left(x_{1},[f \leq s]\right) \leq k^{\prime}\left(r_{1}-s\right) .
$$

The set $\mathcal{A}$ is nonempty (it contains the one-element family $\left\{\left(x_{1}, r_{1}\right)\right\}$ ) and can be ordered by the inclusion relation (that is, $\mathcal{J}_{1} \preceq \mathcal{J}_{2}$ if, and only if, $\mathcal{J}_{1} \subset \mathcal{J}_{2}$ ). Under this relation $\mathcal{A}$ becomes a totally ordered set: every totally ordered chain in $\mathcal{A}$ has an upper bound in $\mathcal{A}$ (its union). Thus, by the Zorn lemma, there exists a maximal element $\mathcal{M}=\left\{\left(x_{i}, r_{i}\right)\right\}_{i \in I}$ in $\mathcal{A}$.

Claim 2. Any maximal element $\mathcal{M}=\left\{\left(x_{i}, r_{i}\right)\right\}_{i \in I}$ of $\mathcal{A}$ satisfies

$$
r^{*}=\inf _{i \in I} r_{i} \leq 0 .
$$

Proof of Claim 2. Let us assume, towards a contradiction, that (13) is not true, i.e. $r^{*}>0$. Let us first assume that there exists $j \in I$ such that $r^{*}=r_{j}$. Define $r^{-}:=r_{j}^{-}<r_{j}$ and $x_{j}^{-}=x^{-} \in\left[f=r^{-}\right]$as specified in Claim 1 and consider the family $\mathcal{M}_{1}=\mathcal{M} \cup\left\{\left(x^{-}, r^{-}\right)\right\}$. Then $\mathcal{M}_{1}$ clearly complies with $\left(\mathrm{P}_{1}\right)$. To see that $\mathcal{M}_{1}$ satisfies $\left(\mathrm{P}_{2}\right)$, simply observe that for each $i \in I$,

$$
d\left(x^{-}, x_{i}\right) \leq d\left(x^{-}, x_{j}\right)+d\left(x_{j}, x_{i}\right) \leq k^{\prime}\left(r_{i}-r^{-}\right) .
$$

Let $s \in\left[r^{-}, r_{j}\right]$. By using the properties of the couple $\left(x^{-}, r^{-}\right)$, one obtains $\operatorname{dist}\left(x_{1},[f \leq s]\right) \leq \operatorname{dist}\left(x_{1}, x_{j}\right)+\operatorname{dist}\left(x_{j},[f \leq s]\right) \leq k^{\prime}\left(r_{1}-r_{j}\right)+k^{\prime}\left(r_{j}-s\right) \leq k^{\prime}\left(r_{1}-s\right)$.

This means that $\mathcal{M}_{1} \in \mathcal{A}$, which contradicts the maximality of $\mathcal{M}$.

Thus it remains to treat the case when the infimum $r^{*}$ is not attained. Let us take any decreasing sequence $\left\{r_{i_{n}}\right\}_{n \geq 1}, i_{n} \in I$ satisfying $r_{i_{1}}=r_{1}$ and $r_{i_{n}} \searrow r^{*}$. For simplicity the sequences $\left\{r_{i_{n}}\right\}_{n \geq 1}$ and $\left\{x_{i_{n}}\right\}_{n \geq 1}$ will be denoted, respectively, by $\left\{r_{n}\right\}_{n \geq 1}$ and $\left\{x_{n}\right\}_{n \geq 1}$. Applying $\left(\mathrm{P}_{2}\right)$ we obtain

$$
d\left(x_{n}, x_{n+m}\right) \leq k^{\prime}\left(r_{n}-r_{n+m}\right) .
$$

It follows that $\left\{x_{n}\right\}_{n \geq 1}$ is a Cauchy sequence; thus it converges to some $x^{*}$. Taking the limit as $m \rightarrow+\infty$ in (14) we deduce that $d\left(x_{n}, x^{*}\right) \leq k^{\prime}\left(r_{n}-r^{*}\right)$, for all $n \in \mathbb{N}^{*}$. For any $i \in I$, there exists $n$ such that $r_{n}<r_{i}$ and therefore

$$
\operatorname{dist}\left(x^{*}, x_{i}\right) \leq d\left(x^{*}, x_{n}\right)+d\left(x_{n}, x_{i}\right) \leq k^{\prime}\left(r_{i}-r^{*}\right) \leq k^{\prime}\left(r_{i}-f\left(x^{*}\right)\right),
$$

where the last inequality follows from the lower semicontinuity of $f$. Set $f\left(x^{*}\right)=$ $\rho^{*} \leq r^{*}$ and $\mathcal{M}_{1}=\mathcal{M} \cup\left\{\left(x^{*}, \rho^{*}\right)\right\}$. Since the infimum is not attained $\operatorname{in} \inf \left\{r_{i}: i \in\right.$ $I\}$ the family $\mathcal{M}_{1}$ satisfies $\left(\mathrm{P}_{1}\right)$. Further by using (15), we see that $\mathcal{M}_{1}$ complies 
also with $\left(\mathrm{P}_{2}\right)$. Take $s \in\left[\rho^{*}, r^{*}\right]$. Since $x^{*} \in[f \leq s]$, we have

$$
\operatorname{dist}\left(x_{1},[f \leq s]\right) \leq \operatorname{dist}\left(x_{1}, x^{*}\right) \leq k^{\prime}\left(r_{1}-r^{*}\right) \leq k^{\prime}\left(r_{1}-s\right) .
$$

Hence $\mathcal{M}_{1}$ belongs to $\mathcal{A}$, which contradicts the maximality of $\mathcal{M}$.

The desired implication follows by letting $k^{\prime} \rightarrow k$. This completes the proof.

Remark 3 (Sublevel mapping and Lipschitz continuity). It is straightforward to see that statement (ii) above is equivalent to the "Lipschitz continuity" (see (7)) of the sublevel set mapping

$$
\left\{\begin{array}{cll}
\left(0, r_{0}\right) & \rightrightarrows X \\
r & \longmapsto & {[f \leq r]}
\end{array}\right.
$$

for the Hausdorff "metric" given in (7). Note that $F^{-1}$ is exactly the sublevel mapping given above, and thus in this context the Lipschitz continuity of $F^{-1}$ is equivalent to the Aubin property of $F^{-1}$; see [24, 32].

2.2. Metric regularity and KŁ-inequality. As a consequence of Theorem 2 and Remark 3, we have the following result.

Corollary 4 (KL-inequality and sublevel set mapping). Let $f: X \longrightarrow \mathbb{R} \cup\{+\infty\}$ be a lower semicontinuous function defined on a complete metric space $X$ and $\varphi \in$ $\mathcal{K}\left(0, r_{0}\right)$ (see (8) $)$. Assume that $\left[0<f<r_{0}\right] \neq \emptyset$. Then the following assertions are equivalent:

(i) the multivalued mapping

$$
\left\{\begin{aligned}
X & \rightrightarrows \mathbb{R} \\
x & \mapsto[(\varphi \circ f)(x),+\infty)
\end{aligned}\right.
$$

is $k$-metrically regular on $\left[0<f<r_{0}\right] \times\left(0, \varphi\left(r_{0}\right)\right)$;

(ii) for all $r_{1}, r_{2} \in\left(0, r_{0}\right)$,

$$
\operatorname{Dist}\left(\left[f \leq r_{1}\right],\left[f \leq r_{2}\right]\right) \leq k\left|\varphi\left(r_{1}\right)-\varphi\left(r_{2}\right)\right| ;
$$

(iii) for all $x \in\left[0<f<r_{0}\right]$,

$$
|\nabla(\varphi \circ f)|(x) \geq \frac{1}{k}
$$

If any of the above assertions holds, the sublevel set $[f \leq 0]$ is nonempty.

It might be useful to observe the following:

Remark 5 (Change of metric). Shrinking $r_{0}$ if necessary, we can assume that any $\varphi \in \mathcal{K}\left(0, r_{0}\right)$ can be extended continuously to an increasing function still denoted $\varphi: \mathbb{R}_{+} \rightarrow \mathbb{R}_{+}$. Set $d_{\varphi}(r, s)=|\varphi(r)-\varphi(s)|$ for any $r, s \in \mathbb{R}_{+}$and assume that $\mathbb{R}_{+}$ is endowed with the metric $d_{\varphi}$. (The interested reader might want to compare this change of metric with the one given in [16, Theorem 3.1].) Endowing $\mathbb{R}_{+}$with this new metric, assertions (i), (ii) and (iii) can be reformulated very simply:

$\left(i^{\prime}\right)$ The multivalued mapping

$$
\left\{\begin{array}{rll}
X & \rightrightarrows \mathbb{R}_{+} \\
x & \mapsto[f(x),+\infty)
\end{array}\right.
$$

is $k$-metrically regular on $\left[0<f<r_{0}\right] \times\left(0, r_{0}\right)$.

(ii') The sublevel mapping

$$
\mathbb{R}_{+} \ni r \mapsto[f \leq r]
$$

is $k$-Lipschitz continuous on $\left(0, r_{0}\right)$. 
(iii') For all $x \in\left[0<f<r_{0}\right]$,

$$
\left|\nabla_{\varphi} f\right|(x) \geq \frac{1}{k}
$$

where $\left|\nabla_{\varphi} f\right|$ denotes the strong slope of the restricted function $\bar{f}:[0<f] \rightarrow$ $\left[\mathbb{R}_{+}, d_{\varphi}\right]$. A function satisfying this last property may be called essentially metrically regular.

Given a lower semicontinuous function $f: X \longrightarrow \mathbb{R} \cup\{+\infty\}$ we say that $f$ is strongly slope-regular if for each point $x$ in its domain $\operatorname{dom} f$ one has

$$
|\nabla f|(x)=|\nabla(-f)|(x) .
$$

Note that, according to the above definition, all $C^{1}$ functions in a Banach space are strongly slope-regular.

Proposition 6 (Level mapping and Lipschitz continuity). Let $X$ be a complete metric space with connected balls. Assume that $f: X \rightarrow \mathbb{R}$ is continuous, strongly slope-regular and $\left(0, r_{0}\right) \subset f(X)$. Then any of the assertions (i)-(iii) of Theorem 2 is equivalent to the fact that the level set application

$$
\left\{\begin{aligned}
\mathbb{R} & \rightrightarrows X \\
r & \mapsto[f=r]
\end{aligned}\right.
$$

is Lipschitz continuous on $\left(0, r_{0}\right)$ with respect to the Hausdorff metric and implies that the level sets $[f=0]$ and $\left[f=r_{0}\right]$ are nonempty.

Proof. For any $r \in\left(0, r_{0}\right)$ and $x \in[f>r]$ we have dist $(x,[f \leq r])=\operatorname{dist}(x,[f=$ $r])$. To see this, note that for every connected set $B$, such that $B \cap[f \leq r] \neq \emptyset$ and $x \in B$ we have $B \cap[f=r] \neq \emptyset$. The result now follows by applying Theorem 2 twice. (The details are left to the reader.)

Let us finally state the following important corollary.

Corollary 7 (KŁ-inequality and level set mapping). Let $f: X \rightarrow \mathbb{R}$ be as in Proposition [6, and $\varphi \in \mathcal{K}\left(0, r_{0}\right)$ (recall (8) ). The following assumptions are equivalent and imply the nonemptiness of the level set $[f=0]$ :

(i) $\varphi \circ f$ is $k$-metrically regular on $\left[0<f<r_{0}\right] \times\left(0, \varphi\left(r_{0}\right)\right)$;

(ii) for all $r_{1}, r_{2} \in\left(0, r_{0}\right)$,

$$
\operatorname{Dist}\left(\left[f=r_{1}\right],\left[f=r_{2}\right]\right) \leq k\left|\varphi\left(r_{1}\right)-\varphi\left(r_{2}\right)\right| ;
$$

(iii) for all $x \in\left[0<f<r_{0}\right]$,

$$
|\nabla(\varphi \circ f)|(x) \geq \frac{1}{k}
$$

Proof. The corollary follows easily by combining Theorem 2 with Proposition 6 .

\section{KŁ-Inequality in Hilbert spaces}

From now on, we shall work on a real Hilbert space $[H,\langle\cdot, \cdot\rangle]$. Given a vector $x$ in $H$, the norm of $x$ is defined by $\|x\|=\sqrt{\langle x, x\rangle}$ while for any subset $C$ of $H$, we set

$$
\|C\|_{-}=\operatorname{dist}(0, C)=\inf \{\|x\|: x \in C\} \in \mathbb{R} \cup\{+\infty\} .
$$

Note that $C=\emptyset$ implies $\|C\|_{-}=+\infty$. 
3.1. Elements of nonsmooth analysis. Let us first recall the notion of Fréchet subdifferential (see [14, 46]).

Definition 8 (Fréchet subdifferential). Let $f: H \rightarrow \mathbb{R} \cup\{+\infty\}$ be a real-extendedvalued function. We say that $p \in H$ is a (Fréchet) subgradient of $f$ at $x \in \operatorname{dom} f$ if

$$
\liminf _{y \rightarrow x, y \neq x} \frac{f(y)-f(x)-\langle p, y-x\rangle}{\|y-x\|} \geq 0 .
$$

We denote by $\partial f(x)$ the set of Fréchet subgradients of $f$ at $x$ and set $\partial f(x)=\emptyset$ for $x \notin \operatorname{dom} f$. Let us now define the notion of a critical point in variational analysis.

Definition 9 (Critical point/values). (i) A point $x_{0} \in H$ is called critical for the function $f$ if $0 \in \partial f\left(x_{0}\right)$.

(ii) The value $r \in f(\operatorname{dom} f)$ is called a critical value if $[f=r]$ contains at least one critical point.

In this section we mainly deal with semiconvex functions. (The reader should be aware that the terminology is not yet completely fixed in this area, so that the notion of semiconvex function may vary slightly from one author to another.)

Definition 10 (Semiconvexity). A proper lower semicontinuous function $f$ is called semiconvex (or convex up to a square) if for some $\alpha>0$ the function

$$
x \longmapsto f(x)+\frac{\alpha}{2}\|x\|^{2}
$$

is convex.

Remark 11. (i) For each $x \in H, \partial f(x)$ is a (possibly empty) closed convex subset of $H$ and $\partial f(x)$ is nonempty for $x \in \operatorname{int} \operatorname{dom} f$.

(ii) It is straightforward from the above definition that the multivalued operator $x \longmapsto \partial f(x)+\alpha x$ is (maximal) monotone (see [53, Definition 12.5] for the definition).

(iii) For general properties of semiconvex functions, see [2, 13. Let us mention that Definition 10 is equivalent to the fact that

$$
f(y)-f(x) \geq\langle p, y-x\rangle-\alpha\|x-y\|^{2},
$$

for all $x, y \in H$ and all $p \in \partial f(x)$ (where $\alpha>0$ ).

(iv) According to Definition 10, semiconvex functions are contained in several important classes of (nonsmooth) functions, as for instance $\phi$-convex functions ([21]), weakly convex functions (4 4 ) and primal-lower-nice functions (44]). Although an important part of the forthcoming results is extendable to more general classes, we have decided to avoid full generality for the sake of simplicity of the presentation.

(v) It is easily seen that every $C^{2}$ function $f$ with bounded second derivative is semiconvex. In this case $\partial f$ is simply reduced to the derivative of $f$.

Given a real-extended-valued function $f$ on $H$, we define the remoteness (i.e., distance to zero) of its subdifferential $\partial f$ at $x \in H$ as follows:

(remoteness)

$$
\|\partial f(x)\|_{-}=\inf _{p \in \partial f(x)}\|p\|=\operatorname{dist}(0, \partial f(x)) .
$$

Remark 12 (Minimal norm). (i) If $\partial f(x) \neq \emptyset$, the infimum in the above definition is achieved since $\partial f(x)$ is a nonempty closed convex set. If we define $\partial^{0} f(x)$ as the 
projection of 0 on the closed convex set $\partial f(x)$ we of course have

$$
\|\partial f(x)\|_{-}=\left\|\partial^{0} f(x)\right\| .
$$

Some properties of $H \ni x \mapsto\|\partial f(x)\|_{-}$are given in Section 5 (Annex).

(ii) If $f$ is a semiconvex function, then $\|\partial f(x)\|_{-}$coincides with the notion of strong slope $|\nabla f|(x)$ introduced in (6); ; see Lemma 43 (Annex).

3.2. Subgradient curves: Basic properties. We recall that $f: H \rightarrow \mathbb{R} \cup$ $\{+\infty\}$ is a proper lower semicontinuous semiconvex function. The purpose of this subsection is to recall the main properties of the trajectories (subgradient curves) of the corresponding differential inclusion:

$$
\left\{\begin{array}{l}
\dot{\chi}_{x}(t) \in-\partial f\left(\chi_{x}(t)\right) \quad \text { a.e. on }(0,+\infty), \\
\chi_{x}(0)=x \in \operatorname{dom} f .
\end{array}\right.
$$

The following statement aggregates useful results concerning existence and uniqueness of solutions. These results are essentially known even for a more general class of functions (see [44, Theorem 2.1, Proposition 2.14, Theorem 3.3] for instance for the class of primal-lower-nice functions). It should also be noticed that the integration of measurable curves (and thus, of the forthcoming absolutely continuous curves) of the form $\mathbb{R} \ni t \rightarrow \gamma(t) \in H$ relies on the Bochner integration/measurability theory (basic properties can be found in [11]).

Theorem 13 (Subgradient curves). For every $x \in \operatorname{dom} f$ there exists a unique absolutely continuous curve (called a subgradient curve) $\chi_{x}:[0,+\infty) \rightarrow H$ that satisfies

$$
\left\{\begin{array}{l}
\dot{\chi}_{x}(t) \in-\partial f\left(\chi_{x}(t)\right) \quad \text { a.e. on }(0,+\infty), \\
\chi_{x}(0)=x \in \operatorname{dom} f .
\end{array}\right.
$$

Moreover the curve satisfies:

(i) $\chi_{x}(t) \in \operatorname{dom} \partial f$ for all $t \in(0,+\infty)$.

(ii) For all $t>0$, the right derivative $\dot{\chi}_{x}\left(t^{+}\right)$of $\chi_{x}$ is well defined and satisfies

$$
\dot{\chi}_{x}\left(t^{+}\right)=-\partial^{0} f\left(\chi_{x}(t)\right) .
$$

In particular $\dot{\chi}_{x}(t)=-\partial^{0} f\left(\chi_{x}(t)\right)$, for almost all $t$.

(iii) The mapping $t \mapsto\left\|\partial f\left(\chi_{x}(t)\right)\right\|_{-}$is right-continuous at each $t \in(0,+\infty)$.

(iv) The function $t \longmapsto f\left(\chi_{x}(t)\right)$ is nonincreasing and continuous on $[0,+\infty)$. Moreover, for all $t, \tau \in[0,+\infty)$ with $t \leq \tau$, we have

$$
f\left(\chi_{x}(t)\right)-f\left(\chi_{x}(\tau)\right) \geq \int_{t}^{\tau}\left\|\dot{\chi}_{x}(u)\right\|^{2} d u,
$$

and equality holds if $t>0$.

(v) The function $t \longmapsto f\left(\chi_{x}(t)\right)$ is Lipschitz continuous on $[\eta,+\infty)$ for any $\eta>0$. Moreover

$$
\frac{d}{d t} f\left(\chi_{x}(t)\right)=-\left\|\dot{\chi}_{x}(t)\right\|^{2} \text { a.e on }(\eta,+\infty) .
$$

Proof. We recall that the subgradient curves of a semiconvex function $f$ are defined for all $t \geq 0$. The only assertion that does not appear explicitly in 44] is the continuity of the function $f \circ \chi_{x}$ at $t=0$ when $x \in \operatorname{dom} f \backslash \operatorname{dom} \partial f$, but this is 
an easy consequence of the fact that $f$ is lower semicontinuous, $\chi_{x}$ is (absolutely) continuous and $f \circ \chi_{x}$ is decreasing. For the rest of the assertions we refer to 44.

The following result asserts that the semiflow mapping associated with the differential inclusion (20) is continuous. This type of result can be established by standard techniques and therefore is essentially known (see 11, 44 for example). We give here an outline of the proof (in the case that $f$ is semiconvex) for the reader's convenience.

Theorem 14 (Continuity of the semiflow). For any semiconvex function $f$, the semiflow mapping

$$
\left\{\begin{array}{cl}
\mathbb{R}_{+} \times \operatorname{dom} f & \rightarrow H \\
(t, x) & \mapsto \chi_{x}(t)
\end{array}\right.
$$

is (norm) continuous on each subset of the form $[0, T] \times(B(0, R) \cap[f \leq r])$, where $T, R>0$ and $r \in \mathbb{R}$.

Proof. Let us fix $x, y \in \operatorname{dom} f$ and $T>0$. Then for almost all $t \in[0, T]$, there exist $p\left(\chi_{x}(t)\right) \in \partial f\left(\chi_{x}(t)\right)$ and $q\left(\chi_{y}(t)\right) \in \partial f\left(\chi_{y}(t)\right)$ such that

$$
\begin{aligned}
\frac{d}{d t}\left\|\chi_{x}(t)-\chi_{y}(t)\right\|^{2} & =2\left\langle\chi_{x}(t)-\chi_{y}(t), \dot{\chi}_{x}(t)-\dot{\chi}_{y}(t)\right\rangle \\
& =-2\left\langle\chi_{x}(t)-\chi_{y}(t), p\left(\chi_{x}(t)\right)-q\left(\chi_{y}(t)\right)\right\rangle .
\end{aligned}
$$

It follows by (18) that

$$
\frac{d}{d t}\left\|\chi_{x}(t)-\chi_{y}(t)\right\|^{2} \leq 2 \alpha\left\|\chi_{x}(t)-\chi_{y}(t)\right\|^{2},
$$

which implies (using the Grönwall lemma) that for all $0 \leq t \leq T$ we have

$$
\left\|\chi_{x}(t)-\chi_{y}(t)\right\|^{2} \leq \exp (2 \alpha T)\|x-y\|^{2} .
$$

For any $0 \leq t \leq s \leq T$, using the Cauchy-Schwarz inequality and Theorem 13 we deduce that

$$
\left\|\chi_{x}(s)-\chi_{x}(t)\right\| \leq \int_{t}^{s}\left\|\dot{\chi}_{x}(\tau)\right\| d \tau \leq \sqrt{s-t} \sqrt{\int_{s}^{t}\left\|\dot{\chi}_{x}(\tau)\right\|^{2} d \tau} \leq \sqrt{s-t} \sqrt{f(x)} .
$$

The result follows by combining (21) and (22).

Let us introduce the notions of a piecewise absolutely continuous curve and of a piecewise subgradient curve. This latter notion, due to its robustness, will play a central role in our study.

Definition 15. Let $a, b \in[-\infty,+\infty]$ with $a<b$.

(Piecewise absolutely continuous curve) A curve $\gamma:(a, b) \rightarrow H$ is said to be piecewise absolutely continuous if there exists a countable partition of $(a, b)$ into intervals $I_{k}$ such that the restriction of $\gamma$ to each $I_{k}$ is absolutely continuous. (Note that a piecewise absolutely continuous curve is not necessarily continuous.)

(Length of a curve) Let $\gamma:(a, b) \rightarrow H$ be a piecewise absolutely continuous curve. The length of $\gamma$ is defined by

$$
\text { length }[\gamma]:=\int_{a}^{b}\|\dot{\gamma}(t)\| d t .
$$


(Piecewise subgradient curve) Let $T \in(0,+\infty]$. A curve $\gamma:[0, T) \rightarrow H$ is called a piecewise subgradient curve for (20) if there exists a countable partition of $[0, T]$ into (nontrivial) intervals $I_{k}$ such that:

- the restriction $\left.\gamma\right|_{I_{k}}$ of $\gamma$ to each interval $I_{k}$ is a subgradient curve ;

- for each disjoint pair of intervals $I_{k}, I_{l}$, the intervals $f\left(\gamma\left(I_{k}\right)\right)$ and $f\left(\gamma\left(I_{l}\right)\right)$ have at most one point in common.

Note that piecewise subgradient curves are piecewise absolutely continuous. Observe also that subgradient curves satisfy the above definition in a trivial way.

3.3. Characterizations of the KŁ-inequality. In this section we state and prove one of the main results of this work. Let $f: H \rightarrow \mathbb{R} \cup\{+\infty\}$ and $\bar{x} \in[f=0]$. Throughout this section the following assumptions are used:

- There exist $\bar{r}, \bar{\epsilon}>0$ such that

$$
x \in \bar{B}(\bar{x}, \bar{\epsilon}) \cap[0<f \leq \bar{r}] \Longrightarrow 0 \notin \partial f(x) \quad \text { (noncriticality assumption). }
$$

- There exist $\bar{r}, \bar{\epsilon}>0$ such that

$$
\bar{B}(\bar{x}, \bar{\epsilon}) \cap[f \leq \bar{r}] \text { is (norm) compact (local sublevel compactness). }
$$

Remark 16. (i) When 0 is a critical value (for instance when $\bar{x}$ is critical) (23) can be seen as a Sard-type condition.

(ii) Assumption (24) is always satisfied in finite-dimensional spaces, but is also satisfied in several interesting cases involving infinite-dimensional spaces. Here are two elementary examples.

(ii) 1 The (convex) function $f: \ell^{2}(\mathbb{N}) \rightarrow \mathbb{R}$ defined by

$$
f(x)=\sum_{n \geq 1} n^{2} x_{i}^{2}
$$

has compact lower level sets.

(ii) $)_{2}$ Let $g: \mathbb{R} \rightarrow \mathbb{R} \cup\{+\infty\}$ be a proper lower semicontinuous semiconvex function and let $\Phi: L^{2}(\Omega) \rightarrow \mathbb{R} \cup\{+\infty\}$ be as follows ([10]):

$$
\Phi(x)=\left\{\begin{array}{cl}
\frac{1}{2} \int_{\Omega}\|\nabla x\|^{2}+\int_{\Omega} g(x) & \text { if } x \in H^{1}(\Omega), \\
+\infty, & \text { otherwise. }
\end{array}\right.
$$

The above function is a lower semicontinuous semiconvex function and the sets of the form $[\Phi \leq r] \cap B(\bar{x}, R)$ are relatively compact in $L^{2}(\Omega)$ (use the compact embedding theorem of $H^{1}(\Omega) \hookrightarrow L^{2}(\Omega)$ ).

As the forthcoming Theorem 18 shows, the Kurdyka-Łojasiewicz inequality can be characterized by the finiteness of the length of a "talweg" curve, that is, a piecewise absolutely continuous curve that passes through the points of less steepest descent (compare with [18, 19, 37]).

Definition 17 (Talweg/Valley). Let $\bar{x} \in[f=0]$ (which may or may not be a critical point of $f$ ) and assume that (23) holds for some $\bar{r}, \bar{\epsilon}>0$. Let $D$ be any closed bounded set that contains $B(\bar{x}, \bar{\epsilon}) \cap[0<f \leq \bar{r}]$. For any $R>1$ the $R$-valley $\mathcal{V}_{R}^{D}(\cdot)$ of $f$ around $\bar{x}$ is defined as follows:

$$
\mathcal{V}_{R}^{D}(r)=\left\{x \in[f=r] \cap D:\|\partial f(x)\|_{-} \leq R \inf _{y \in[f=r] \cap D}\|\partial f(y)\|_{-}\right\} \text {for all } r \in(0, \bar{r}] .
$$


A selection $\theta:(0, \bar{r}] \rightarrow H$ of $\mathcal{V}_{R}^{D}$, i.e. a curve such that $\theta(r) \in \mathcal{V}_{R}^{D}(r), \forall r \in(0, \bar{r}]$, is called an $R$-talweg or simply a talweg.

We are ready to state the main result of this work.

Theorem 18 (Subgradient inequality - local characterization). Let $f: H \rightarrow \mathbb{R} \cup$ $\{+\infty\}$ be a lower semicontinuous semiconvex function, let $\bar{x} \in[f=0]$ and assume (23), (24) hold for some $\bar{\epsilon}>0$ and $\bar{r}>0$. The following statements are equivalent:

(i) [Kurdyka-Eojasiewicz inequality $]$ There exist $r_{0} \in(0, \bar{r}), \epsilon \in(0, \bar{\epsilon})$ and $\varphi \in \mathcal{K}\left(0, r_{0}\right)$ such that

$$
\|\partial(\varphi \circ f)(x)\|_{-} \geq 1, \quad \text { for all } x \in \bar{B}(\bar{x}, \epsilon) \cap\left[0<f \leq r_{0}\right] .
$$

(ii) [Length boundedness of subgradient curves] There exist $r_{0} \in(0, \bar{r}), \epsilon \in$ $(0, \bar{\epsilon})$ and a strictly increasing continuous function $\sigma:\left[0, r_{0}\right] \rightarrow[0,+\infty)$ with $\sigma(0)=$ 0 such that for all subgradient curves $\chi_{x}$ of (20) satisfying $\chi_{x}([0, T)) \subset \bar{B}(\bar{x}, \epsilon) \cap[0<$ $\left.f \leq r_{0}\right](T \in(0,+\infty])$ we have

$$
\int_{0}^{T}\left\|\dot{\chi}_{x}(t)\right\| d t \leq \sigma(f(x))-\sigma\left(f\left(\chi_{x}(T)\right)\right) .
$$

(iii) [Piecewise subgradient curves have finite length] There exist $r_{0} \in$ $(0, \bar{r}), \epsilon \in(0, \bar{\epsilon})$ and $M>0$ such that for all piecewise subgradient curves $\gamma$ : $[0, T) \rightarrow H(T \in(0,+\infty])$ of (20) we have

$\gamma([0, T)) \subset \bar{B}(\bar{x}, \epsilon) \cap\left[0<f \leq r_{0}\right] \quad \Longrightarrow \quad \operatorname{length}\left[\left.\gamma\right|_{(0, T)}\right]=\int_{0}^{T}\|\dot{\gamma}(\tau)\| d \tau<M$.

(iv) [Talwege of finite length] For every $R>1$, there exist $r_{0} \in(0, \bar{r}), \epsilon \in$ $(0, \bar{\epsilon})$, a closed bounded subset $D$ containing $B(\bar{x}, \epsilon) \cap\left[0<f \leq r_{0}\right]$ and a piecewise absolutely continuous curve $\theta:\left(0, r_{0}\right] \rightarrow H$ of finite length which is a selection of the valley $\mathcal{V}_{R}^{D}(r)$, that is,

$$
\theta(r) \in \mathcal{V}_{R}^{D}(r), \text { for all } r \in\left(0, r_{0}\right] .
$$

(v) $[$ Integrability condition $]$ There exist $r_{0} \in(0, \bar{r})$ and $\epsilon \in(0, \bar{\epsilon})$ such that the function

$$
u(r)=\frac{1}{\inf _{x \in \bar{B}(\bar{x}, \epsilon) \cap[f=r]}\|\partial f(x)\|_{-}}, \quad r \in\left(0, r_{0}\right]
$$

is finite-valued and belongs to $L^{1}\left(0, r_{0}\right)$. (We recall that $\inf \emptyset=+\infty$ and $1 / \infty=$ 0.)

Remark 19. (i) As it clearly appears from the proof, statement (iv) can be replaced by the following equivalent assertion:

$\left(i v^{\prime}\right)$ There exist $R>1, r_{0} \in(0, \bar{r}), \epsilon \in(0, \bar{\epsilon})$, a closed bounded subset $D$ containing $B(\bar{x}, \epsilon) \cap\left[0<f \leq r_{0}\right]$ and a piecewise absolutely continuous curve $\theta:\left(0, r_{0}\right] \rightarrow H$ of finite length which is a selection of the valley $\mathcal{V}_{R}^{D}(r)$.

(ii) The compactness assumption (24) is only used in the proofs of $(i i i) \Rightarrow(i i)$ and $(i i) \Rightarrow(i v)$. Hence if this assumption is removed, we still have

$$
(i v) \Longrightarrow\left(i v^{\prime}\right) \Longrightarrow(v) \Longleftrightarrow(i) \Longrightarrow(i i) \Longrightarrow(i i i)
$$

(iii) Note that (i) implies condition (23). This follows immediately from the chain rule (see Annex, Lemma 44). 
(iv) The interesting case in Theorem 18 (and thus in Definition 17) is when $\bar{x}$ is a critical point. In this case (26) can be interpreted as a desingularization of $f$ around $\bar{x}$.

Proof of Theorem [18, [(i) $\Rightarrow\left(\right.$ ii)] Let $\epsilon, r_{0}, \varphi$ be as in (i) such that (26) holds. Further let $\chi_{x}$ be a subgradient curve of (20) for $x \in\left[0<f \leq r_{0}\right]$ and assume that $\chi_{x}([0, T)) \subset \bar{B}(\bar{x}, \epsilon) \cap\left[0<f \leq r_{0}\right]$ for some $T>0$.

Let us first assume that $x \in \operatorname{dom} \partial f$. Since $\varphi$ is $C^{1}$ on $\left(0, r_{0}\right)$, by Theorem [13(v) and Lemma 44 (Annex) we deduce that the curve $t \mapsto \varphi\left(f\left(\chi_{x}(t)\right)\right)$ is absolutely continuous with derivative

$$
\frac{d}{d t}\left(\varphi \circ f \circ \chi_{x}\right)(t)=-\varphi^{\prime}\left(f\left(\chi_{x}(t)\right)\right)\left\|\dot{\chi}_{x}(t)\right\|^{2} \text { a.e. on }(0, T) .
$$

Integrating both terms on the interval $(0, T)$ and recalling (26), $\chi_{x}(0)=x$ we get

$$
\begin{aligned}
\varphi(f(x))-\varphi\left(f\left(\chi_{x}(T)\right)\right) & =-\int_{0}^{T} \frac{d}{d t}\left(\varphi \circ f \circ \chi_{x}\right)(t) d t \\
& =\int_{0}^{T} \varphi^{\prime}\left(f\left(\chi_{x}(t)\right)\left\|\dot{\chi}_{x}(t)\right\|^{2} d t \geq \int_{0}^{T}\left\|\dot{\chi}_{x}(t)\right\| d t .\right.
\end{aligned}
$$

Thus (ii) holds true for $\sigma:=\varphi$ and for all subgradient curves starting from points in $\operatorname{dom} \partial f$. Now let $x \in \operatorname{dom} f \backslash \operatorname{dom} \partial f$ and fix any $\delta \in(0, T)$. Since $\chi_{x}([\delta, T]) \subset$ dom $\partial f$ we deduce from the above that

$$
\int_{\delta}^{T}\left\|\dot{\chi}_{x}(t)\right\| d t \leq \sigma\left(f\left(\chi_{x}(\delta)\right)-\sigma\left(f\left(\chi_{x}(T)\right)\right) .\right.
$$

Thus the result follows by taking $\delta \searrow 0^{+}$and using the continuity of the mapping $t \longmapsto f\left(\chi_{x}(t)\right)$ at 0 (Theorem 13(ii)).

[(ii) $\Rightarrow$ (iii)] Let $\gamma$ be a piecewise subgradient curve as in (iii) and let $I_{k}$ be an associated partition of $[0, T]$ (cf. Definition[15). Let $\left\{a_{k}\right\}$ and $\left\{b_{k}\right\}$ be two sequences of real numbers such that int $I_{k}=\left(a_{k}, b_{k}\right)$. Since the restriction $\left.\gamma\right|_{I_{k}}$ of $\gamma$ onto $I_{k}$ is a subgradient curve, applying (ii) on $\left(a_{k}, b_{k}\right)$ we get

$$
\text { length }\left[\left.\gamma\right|_{I_{k}}\right] \leq \sigma\left(f\left(\gamma\left(a_{k}\right)\right)\right)-\sigma\left(f\left(\gamma\left(b_{k}\right)\right)\right) .
$$

Let $m$ be an integer and $I_{k_{1}}, \ldots, I_{k_{m}}$ a finite subfamily of the partition. We may assume that these intervals are ordered as follows: $0 \leq a_{k_{1}} \leq b_{k_{1}} \leq \cdots \leq a_{k_{m}} \leq$ $b_{k_{m}}$. Hence

$$
\sum_{1}^{m}\left[\sigma\left(f\left(\gamma\left(a_{k_{i}}\right)\right)\right)-\sigma\left(f\left(\gamma\left(b_{k_{i}}\right)\right)\right)\right] \leq \sigma\left(f\left(\gamma\left(a_{k_{1}}\right)\right)\right) \leq \sigma\left(r_{0}\right) .
$$

Thus the family $\left\{\sigma\left(f\left(\gamma\left(a_{k}\right)\right)\right)-\sigma\left(f\left(\gamma\left(b_{k}\right)\right)\right)\right\}$ is summable; hence using the definition of the Bochner integral (see [11])

$$
\text { length }[\gamma]=\sum_{k \in \mathbb{N}} \text { length }\left[\left.\gamma\right|_{I_{k}}\right] \leq \sigma\left(r_{0}\right) .
$$

[(iii) $\Rightarrow($ ii) $]$ Let $\epsilon, r_{0}$ be as in (iii), pick any $r, r^{\prime}$ such that $0 \leq r^{\prime}<r \leq r_{0}$ and denote by $\Gamma_{r^{\prime}, r}$ the (nonempty) set of piecewise subgradient curves $\gamma:[0, T) \rightarrow H$ (where $T \in(0,+\infty])$ such that

$$
\gamma([0, T)) \subset \bar{B}(\bar{x}, \epsilon) \cap\left[r^{\prime}<f \leq r\right] .
$$


Note that, by Theorem 13(iv) and Proposition 42(iii), $T=+\infty$ is possible only when 0 is a critical value and $r^{\prime}=0$. Set further

$$
\psi\left(r^{\prime}, r\right):=\sup _{\gamma \in \Gamma_{r^{\prime}, r}} \text { length }[\gamma] \quad \text { and } \quad \sigma(r):=\psi(0, r) .
$$

Note that (iii) guarantees that $\psi$ and $\sigma$ have finite values. We can easily deduce from Definition 15 that

$$
\psi\left(0, r^{\prime}\right)+\psi\left(r^{\prime}, r\right)=\psi(0, r) .
$$

Thus for each $x \in \bar{B}(\bar{x}, \epsilon) \cap\left[0<f \leq r_{0}\right]$ and $T>0$ such that $\chi_{x}([0, T]) \subset$ $B(\bar{x}, \epsilon) \cap\left[0<f \leq r_{0}\right]$, we have

$$
\int_{0}^{T}\left\|\dot{\chi}_{x}(\tau)\right\| d \tau+\sigma\left(f\left(\chi_{x}(T)\right) \leq \sigma(f(x)) .\right.
$$

Since the function $\sigma$ is nonnegative and increasing it can be extended continuously at 0 by setting $\sigma(0)=\lim _{t \downarrow 0} \sigma(t) \geq 0$. Since the property (28) remains valid if we replace $\sigma(\cdot)$ by $\sigma(\cdot)-\sigma(0)$, there is no loss of generality to assume $\sigma(0)=0$.

To conclude it suffices to establish the continuity of $\sigma$ on $\left(0, r_{0}\right]$. Fix $\tilde{r}$ in $\left(0, r_{0}\right)$ and take a subgradient curve $\chi:[0, T) \rightarrow H$ satisfying $\chi([0, T)) \subset \bar{B}(\bar{x}, \epsilon) \cap\left[f \leq r_{0}\right]$, where $T \in(0,+\infty]$. Set $f(\chi(0))=r$ and $\lim _{t \rightarrow T} f(\chi(t))=r^{\prime}$ and assume that $\tilde{r} \leq r^{\prime} \leq r \leq r_{0}$.

From Theorem 13(iv) and Proposition 42 (iii) (Annex), we deduce that $T<+\infty$ so that $\chi([0, T]) \subset \bar{B}(\bar{x}, \epsilon) \cap\left[r^{\prime} \leq f \leq r\right]$. Using assumption (23) together with Theorem [13 (i), (v), we deduce that the absolutely continuous function $f \circ \chi$ : $[0, T] \rightarrow\left[r^{\prime}, r\right]$ is invertible and

$$
\frac{d}{d \rho}[f \circ \chi]^{-1}(\rho)=\frac{-1}{\| \dot{\chi}\left([f \circ \chi]^{-1}(\rho) \|^{2}\right.} \geq \frac{-1}{\inf _{x \in \bar{B}(\bar{x}, \epsilon) \cap\left[\tilde{r} \leq f \leq r_{0}\right]}\|\partial f(x)\|_{-}^{2}}:=-K,
$$

for almost all $\rho \in\left(r, r^{\prime}\right)$. By Proposition 42 (iii) (Annex) we get that $K<+\infty$ and therefore the function $\rho \longmapsto[f \circ \chi]^{-1}(\rho)$ is Lipschitz continuous with constant $K$ on $\left[r^{\prime}, r\right]$. Using the Cauchy-Schwarz inequality and Theorem[13(iv) we obtain

$$
\begin{aligned}
\text { length }[\chi] & =\int_{0}^{T}\|\dot{\chi}\| \leq \sqrt{T} \sqrt{\int_{0}^{T}\|\dot{\chi}\|^{2}} \\
& =\sqrt{[f \circ \chi]^{-1}(r)-[f \circ \chi]^{-1}\left(r^{\prime}\right)} \sqrt{\int_{0}^{T}\|\dot{\chi}\|^{2}} \\
& \leq \sqrt{K\left(r-r^{\prime}\right)} \sqrt{r-r^{\prime}}=\sqrt{K}\left(r-r^{\prime}\right) .
\end{aligned}
$$

This last inequality implies that each piecewise subgradient curve $\gamma:[0, T) \rightarrow H$ such that $\gamma([0, T)) \subset \bar{B}(\bar{x}, \epsilon) \cap\left[r^{\prime} \leq f \leq r\right]$ satisfies

$$
\text { length }[\gamma] \leq \sqrt{K}\left(r-r^{\prime}\right) ;
$$

thus using (27) we obtain $\sigma(r)-\sigma\left(r^{\prime}\right) \leq \sqrt{K}\left(r-r^{\prime}\right)$, which yields the continuity of $\sigma$.

$\left[(i i) \Rightarrow(\right.$ iv) $]$ Let us assume that (ii) holds true for $\epsilon$ and $r_{0}$. In a first step we establish the existence of a closed bounded subset $D$ of $\left[0<f \leq r_{0}\right]$ satisfying

$$
x \in D, t \geq 0, f\left(\chi_{x}(t)\right)>0 \Rightarrow \chi_{x}(t) \in D .
$$


Let $r_{0} \geq r_{1}>0$ be such that $\sigma\left(r_{1}\right)<\epsilon / 3$ and let us set

$$
\begin{aligned}
D:=\left\{y \in \bar{B}(\bar{x}, \epsilon) \cap\left[0<f \leq r_{1}\right]:\right. & \exists x \in \bar{B}(\bar{x}, \epsilon / 3) \cap\left[0<f \leq r_{1}\right], \\
& \left.\exists t \geq 0 \text { such that } \chi_{x}(t)=y\right\} .
\end{aligned}
$$

Let us first show that $D$ complies with property (30). It suffices to establish that

$$
x \in \bar{B}(\bar{x}, \epsilon / 3) \cap\left[0<f \leq r_{1}\right], t \geq 0, f\left(\chi_{x}(t)\right)>0 \Rightarrow \chi_{x}(t) \in D .
$$

To this end, fix $x \in \bar{B}(\bar{x}, \epsilon / 3) \cap\left[0<f \leq r_{1}\right]$. By continuity of the flow, we observe that for small $t>0$ we have $\chi_{x}(t) \in \bar{B}(\bar{x}, \epsilon)$ while for all $t \geq 0$ such that $\chi_{x}([0, t]) \subset \bar{B}(\bar{x}, \epsilon)$ with $f\left(\chi_{x}(t)\right)>0$, assumption (ii) yields

$\left\|\chi_{x}(t)-\bar{x}\right\| \leq\left\|\chi_{x}(t)-x\right\|+\|x-\bar{x}\| \leq \int_{0}^{t}\left\|\dot{\chi}_{x}(\tau)\right\| d \tau+\epsilon / 3 \leq \sigma\left(r_{1}\right)+\epsilon / 3 \leq 2 \epsilon / 3$.

Thus $D$ satisfies (30) and $\bar{B}(\bar{x}, \epsilon / 3) \cap\left[f \leq r_{1}\right] \subset D$.

Let us now prove that $D$ is (relatively) closed in $\left[0<f \leq r_{1}\right]$. Let $\left\{y_{k}\right\}_{k \geq 1}$ be a sequence in $D$ converging to some $y$ with $f(y) \in\left(0, r_{1}\right]$. Then there exist sequences $\left\{x_{n}\right\}_{n} \subset \bar{B}(\bar{x}, \epsilon / 3) \cap\left[0<f \leq r_{1}\right]$ and $\left\{t_{n}\right\}_{n} \subset \mathbb{R}_{+}$such that $\chi_{x_{n}}\left(t_{n}\right)=y_{n}$. Since $f$ is lower semicontinuous, there exists $n_{0} \in \mathbb{N}$ and $\eta>0$ such that $f\left(y_{n}\right)>\eta$ for all $n \geq n_{0}$. By Theorem 13(ii),(iv), (23) and Proposition 42(iii) (Annex), we obtain for all $n \geq n_{0}$,

$$
0<t_{n} \inf _{z \in\left[\eta \leq f \leq r_{1}\right] \cap \bar{B}(\bar{x}, \epsilon)}\|\partial f(z)\|_{-}^{2} \leq \int_{0}^{t_{n}}\left\|\dot{\chi}_{x_{n}}(t)\right\|^{2} d t \leq f\left(x_{n}\right) \leq r_{1} .
$$

The above inequality shows that the sequence $\left\{t_{n}\right\}_{n}$ is bounded. Using a standard compactness argument we therefore deduce that, up to an extraction, $x_{n} \rightarrow \tilde{x}$ and $t_{n} \rightarrow \tilde{t}$ for some $\tilde{x} \in \bar{B}(\bar{x}, \epsilon / 3) \cap\left[f \leq r_{1}\right]$ and $\tilde{t} \in \mathbb{R}_{+}$. Theorem 14 (continuity of the semiflow) implies that $y=\chi_{\tilde{x}}(\tilde{t})$ and consequently that $f(\tilde{x}) \geq f(y)>0$, yielding that $y \in D$. This shows that $D$ is (relatively) closed in $\left[0<f \leq r_{0}\right]$.

Now we build a piecewise absolutely continuous curve in the valley. Following the notation of Proposition 42 (Annex) we set

$$
s_{D}(r):=\inf \left\{\|\partial f(x)\|_{-}: x \in D \cap[f=r]\right\},
$$

so that for any $R>1$ the $R$-valley around $\bar{x}$ (cf. Definition [17) is given by

$$
\mathcal{V}_{R}^{D}(r):=\left\{x \in[f=r] \cap D:\|\partial f(x)\|_{-} \leq R s_{D}(r)\right\} .
$$

If $\bar{B}(\bar{x}, \epsilon / 3) \cap[f=r]=\emptyset$ for all $0<r \leq r_{1}$, there is nothing to prove. Otherwise, there exists $0<r_{2} \leq r_{1}$ and $x_{2} \in \bar{B}(\bar{x}, \epsilon / 3) \cap\left[f=r_{2}\right] \subset D$. From Theorem 13 and Proposition 42 (iii) (Annex), we deduce that $\chi_{x_{2}}(t) \in\left[f=f\left(\chi_{x_{2}}(t)\right)\right] \cap D \cap \operatorname{dom} \partial f$ for all $t \geq 0$ such that $\left[f \circ \chi_{x_{2}}\right](t)>0$ and that the inverse function $\left[f \circ \chi_{x_{2}}\right]^{-1}(\cdot)$ is defined on an interval containing $\left(0, r_{2}\right)$. In other words the set $[f=r] \cap D \cap$ $\operatorname{dom} \partial f$ is nonempty for each $r \in\left(0, r_{2}\right)$, which in turn implies that the valley $\mathcal{V}_{R}^{D}$ is nonempty for small positive values of $r$, i.e. $\mathcal{V}_{R}^{D}(r) \neq \emptyset$ for all $r \in\left(0, r_{2}\right)$. With no loss of generality we assume that $\mathcal{V}_{R}^{D}\left(r_{2}\right) \neq \emptyset$.

Further let $R^{\prime} \in(1, R)$ and $x \in\left[f=r_{2}\right] \cap D$ be such that $\|\partial f(x)\|_{-} \leq R^{\prime} s_{D}\left(r_{2}\right)$ (therefore, in particular, $x \in \mathcal{V}_{R}^{D}\left(r_{2}\right)$ ). Take $\rho \in\left(R^{\prime}, R\right)$. Since the mapping $t \longmapsto \| \partial f\left(\chi_{x}(t) \|_{-}\right.$is right-continuous (cf. Theorem[13(iii)), there exists $t_{0}>0$ such that $\| \partial f\left(\chi_{x}(t) \|_{-}<\rho s_{D}\left(r_{2}\right)\right.$ for all $t \in\left(0, t_{0}\right)$. On the other hand $t \longmapsto s_{D}\left(f\left(\chi_{x}(t)\right)\right.$ is lower semicontinuous ( $c f$. Proposition 42-Annex); hence there exists $t_{1} \in\left(0, t_{0}\right)$ such that $R s_{D}\left(f\left(\chi_{x}(t)\right)>\rho s_{D}\left(r_{2}\right)\right.$, for all $t \in\left(0, t_{2}\right)$. Using the continuity of the 
mapping $\chi_{x}(\cdot)$ and the stability property (30), we obtain the existence of $t_{2}>0$ such that

$$
\chi_{x}(t) \in \mathcal{V}_{R}^{D}(f(x(t))) \text { for all } t \in\left[0, t_{2}\right) .
$$

By using arguments similar to those of $[(\mathrm{iii}) \Rightarrow(\mathrm{ii})]$ we define the following absolutely continuous curve:

$$
\left(f \circ \chi_{x}\left(t_{2}\right), r_{2}\right] \ni r \longmapsto \theta(r)=\chi_{x}\left(\left[f \circ \chi_{x}\right]^{-1}(r)\right) \in D \cap[f=r] .
$$

By Proposition 47 based on the Zorn lemma (see Annex), we obtain a piecewise subgradient curve that we still denote by $\theta$, defined on $\left(0, r_{2}\right]$, satisfying $\theta(r) \in$ $\mathcal{V}_{R}^{D}(r)$ for all $r \in\left(0, r_{2}\right]$. Assumption (iii) now yields

$$
\text { length }[\theta]<M<+\infty \text {, }
$$

which completes the proof of the assertion.

[(iv) $\Rightarrow(\mathbf{v})]$ Fix $R>1$ and let $\epsilon, r_{0}$ and $\theta:\left(0, r_{0}\right] \rightarrow H$ be as in (iv). Applying Lemma 44 (Annex), we get

$$
\frac{d}{d r}(f \circ \theta)(r)=1=\langle\dot{\theta}(r), p(r)\rangle \text { a.e. on }\left(0, r_{0}\right], \quad \text { for all } p(r) \in \partial f(\theta(r)) .
$$

Using the Cauchy-Schwarz inequality together with the fact that $D \cap[f=r] \supset$ $\bar{B}(\bar{x}, \epsilon) \cap[f=r]$, we obtain

$$
R\|\dot{\theta}(r)\| \geq u(r)=\frac{1}{\inf _{x \in \bar{B}(\bar{x}, \epsilon) \cap[f=r]}\|\partial f(x)\|_{-}},
$$

for almost all $r \in\left(0, r_{0}\right]$. Since $\theta$ has finite length and $u$ is measurable we deduce that $u \in L^{1}\left(\left(0, r_{0}\right)\right.$.

$[(\mathbf{v}) \Rightarrow(\mathbf{i})]$ Let $\epsilon, r_{0}$ and $u$ be as in $(v)$. From Proposition 42 (Annex) we deduce that $u$ is finite-valued and upper semicontinuous. Applying Lemma 45 (Annex) we obtain a continuous function $\bar{u}:\left(0, r_{0}\right] \rightarrow(0,+\infty)$ such that $\bar{u}(r) \geq u(r)$ for all $r \in\left(0, r_{0}\right]$. Set

$$
\varphi(r)=\int_{0}^{r} \bar{u}(s) d s .
$$

It follows directly that $\varphi(0)=0, \varphi \in C([0, r]) \cap C^{1}\left(0, r_{0}\right)$ and $\varphi^{\prime}(r)>0$ for all $r \in\left(0, r_{0}\right)$. Let $x \in \bar{B}(\bar{x}, \epsilon) \cap[f=r]$ and $q \in \partial(\varphi \circ f)(x)$. From Lemma 44 (Annex) we deduce $p:=\frac{q}{\varphi^{\prime}(r)} \in \partial f(x)$, and therefore

$$
\|q\|=\varphi^{\prime}(r)\left\|\frac{q}{\varphi^{\prime}(r)}\right\| \geq u(r)\|p\| \geq 1 .
$$

The proof is complete.

Under a stronger compactness assumption, Theorem 18 can be reformulated as follows.

Theorem 20 (Subgradient inequality - global characterization). Let $f: H \rightarrow$ $\mathbb{R} \cup\{+\infty\}$ be a lower semicontinuous semiconvex function. Assume that there exists $r_{0}>0$ such that

$$
\left[f \leq r_{0}\right] \text { is (nonempty) compact and } 0 \notin \partial f(x), \forall x \in\left[0<f<r_{0}\right] .
$$

The following propositions are equivalent.

(i) [Kurdyka-Eojasiewicz inequality $]$ There exists $\varphi \in \mathcal{K}\left(0, r_{0}\right)$ such that

$$
\|\partial(\varphi \circ f)(x)\|_{-} \geq 1, \quad \text { for all } x \in\left[0<f<r_{0}\right] .
$$


(ii) [Length boundedness of subgradient curves] There exists an increasing continuous function $\sigma:\left[0, r_{0}\right) \rightarrow[0,+\infty)$ with $\sigma(0)=0$ such that for all subgradient curves $\chi_{x}(\cdot)$ (where $x \in\left[0<f<r_{0}\right]$ ) we have

$$
\int_{0}^{T}\left\|\dot{\chi}_{x}(t)\right\| d t \leq \sigma(f(x))-\sigma\left(f\left(\chi_{x}(T)\right)\right)
$$

whenever $f\left(\chi_{x}(T)\right)>0$.

(iii) [Piecewise subgradient curves have bounded length] There exists $M>$ 0 such that for all piecewise subgradient curves $\gamma:[0, T) \rightarrow H$ such that $\gamma([0, T)) \subset$ $\left[0<f<r_{0}\right]$ we have

$$
\text { length }[\gamma]<M \text {. }
$$

(iv) [Talwege of finite length] For all $R>1$, there exists a piecewise absolutely continuous curve (with countable pieces) $\theta:\left(0, r_{0}\right) \rightarrow \mathbb{R}^{n}$ with finite length such that

$$
\theta(r) \in\left\{x \in[f=r]:\|\partial f(x)\|_{-} \leq R \inf _{y \in[f=r]}\|\partial f(y)\|_{-}\right\}, \quad \text { for all } r \in\left(0, r_{0}\right) .
$$

(v) $[$ Integrability condition $]$ The function $u:\left(0, r_{0}\right) \rightarrow[0,+\infty]$ defined by

$$
u(r)=\frac{1}{\inf _{x \in[f=r]}\|\partial f(x)\|_{-}}, \quad r \in\left(0, r_{0}\right)
$$

is finite-valued and belongs to $L^{1}\left(0, r_{0}\right)$.

(vi) [Lipschitz continuity of the sublevel mapping] There exists $\varphi \in \mathcal{K}\left(0, r_{0}\right)$ such that

$$
\operatorname{Dist}([f \leq r],[f \leq s]) \leq|\varphi(r)-\varphi(s)| \quad \text { for all } r, s \in\left(0, r_{0}\right) .
$$

Proof. The proof is similar to the proof of Theorem 18 and will be omitted. The equivalence between (i) and (vi) is a consequence of Corollary 4

3.4. Application: Convergence of the proximal algorithm. Let us recall the definition of the proximal mapping (see [53, Definition 1.22], for example).

Definition 21 (Proximal mapping). Let $\lambda \in\left(0, \alpha^{-1}\right)$. Then the proximal mapping $\operatorname{prox}_{\lambda}: H \rightarrow H$ is defined by

$$
\operatorname{prox}_{\lambda}(x):=\operatorname{argmin}\left\{f(y)+\frac{1}{2 \lambda}\|y-x\|^{2}\right\}, \forall x \in H .
$$

Remark 22. The assumption that $f$ is semiconvex ( $c f$. Definition 10) ensures that $\operatorname{prox}_{\lambda}$ is well-defined and single-valued: indeed, it implies that the auxiliary function appearing in the aforementioned definition is strictly convex and coercive (see [53], [15] for instance).

Lemma 23 (Subgradient inequality and proximal mapping). Assume that $f: H \rightarrow$ $\mathbb{R} \cup\{+\infty\}$ is a semiconvex function that satisfies the condition (i) of Theorem 20 , Let $x \in\left[0<f<r_{0}\right]$ be such that $f\left(\operatorname{prox}_{\lambda} x\right)>0$. Then

$$
\left\|\operatorname{prox}_{\lambda} x-x\right\| \leq \varphi(f(x))-\varphi\left(f\left(\operatorname{prox}_{\lambda} x\right)\right) .
$$

Proof. Set $x^{+}=\operatorname{prox}_{\lambda}(x), r=f(x)$, and $r^{+}=f\left(x^{+}\right)$. It follows from the definition of $x^{+}$that $0<r^{+} \leq r<r_{0}$. In particular, for every $u \in\left[f \leq r^{+}\right]$we have

$$
\left\|x^{+}-x\right\|^{2} \leq\|u-x\|^{2}+2 \lambda\left[f(u)-r^{+}\right] \leq\|u-x\|^{2} .
$$


Therefore by Corollary 4 (Lipschitz continuity of the sublevel mapping) we obtain

$$
\left\|x^{+}-x\right\|=\operatorname{dist}\left(x,\left[f \leq r^{+}\right]\right) \leq \operatorname{Dist}\left([f \leq r],\left[f \leq r^{+}\right]\right) \leq \varphi(r)-\varphi\left(r^{+}\right) .
$$

The proof is complete.

The above result has an important impact in the asymptotic analysis of the proximal algorithm (see forthcoming Theorem 24). Let us first recall that, given a sequence of positive parameters $\left\{\lambda_{k}\right\}_{k \geq 1} \subset\left(0, \alpha^{-1}\right)$ and $x \in H$ the proximal algorithm is defined as follows:

$$
Y_{x}^{k+1}=\operatorname{prox}_{\lambda_{k}} Y_{x}^{k}, \quad Y_{x}^{0}=x,
$$

or in other words

$$
\left\{Y_{x}^{k+1}\right\}=\operatorname{argmin}\left\{f(u)+\frac{1}{2 \lambda_{k}}\left\|u-Y_{x}^{k}\right\|^{2}\right\}, \quad Y_{x}^{0}=x .
$$

If we assume in addition that inf $f>-\infty$, then for any initial point $x$ the sequence $\left\{f\left(Y_{x}^{k}\right)\right\}$ is decreasing and converges to a real number $l$.

Theorem 24 (Strong convergence of the proximal algorithm). Let $f: H \rightarrow \mathbb{R} \cup$ $\{+\infty\}$ be a semiconvex function which is bounded from below. Let $x \in \operatorname{dom} f$, $\left\{\lambda_{k}\right\}_{k \geq 1} \subset\left(0, \alpha^{-1}\right)$,

$$
y_{k}:=Y_{x}^{k}, \text { for } k \geq 0 \quad(\text { recall (34) }) \quad \text { and } \quad l:=\lim _{k \rightarrow \infty} f\left(y_{k}\right) .
$$

Assume that there exists $k_{0} \geq 0$ and $\varphi \in \mathcal{K}\left(0, f\left(y_{k_{0}}\right)-l\right)$ such that

$$
\|\partial(\varphi \circ[f(\cdot)-l])(x)\|_{-} \geq 1, \quad \text { for all } x \in\left[l<f \leq f\left(y_{k_{0}}\right)\right] .
$$

Then the sequence $\left\{y_{k}\right\}_{k \geq 0}$ converges strongly to $y_{\infty}$ and

$$
\left\|y_{\infty}-y_{k}\right\| \leq \varphi\left(f\left(y_{k}\right)-l\right), \quad \text { for all } k \geq k_{0} .
$$

Proof. Since the sequence $\left\{y_{k}\right\}_{k \geq k_{0}}$ evolves in $l \leq f<f\left(y_{k_{0}}\right)$, Lemma 23 applies. This yields

$$
\sum_{k=p}^{q}\left\|y_{k+1}-y_{k}\right\| \leq \varphi\left(f\left(y_{q+1}\right)-l\right)-\varphi\left(f\left(y_{p}\right)-l\right),
$$

for all integers $k_{0} \leq p \leq q$. This implies that $y_{k}$ converges strongly to $y_{\infty}$ and that inequality (36) holds.

Remark 25 (Step-size). "Surprisingly" enough the step-size sequence $\left\{\lambda_{k}\right\}$ does not appear explicitly in the estimate (36), but it is instead hidden in the sequence of values $\left\{f\left(y_{k}\right)\right\}$. In practice the choice of the step-size parameters $\lambda_{k}$ is however crucial to obtain the convergence of $\left\{f\left(y^{k}\right)\right\}$ to a critical value; standard choices are for example sequences satisfying $\sum \lambda_{k}=+\infty$ or $\lambda_{k} \in\left[\eta, \alpha^{-1}\right)$ for all $k \geq 0$, where $\eta \in\left(0, \alpha^{-1}\right)$; see [15] for more details.

\section{ConveXity And KŁ-INEquality}

In this section, we assume that $f: H \rightarrow \mathbb{R} \cup\{+\infty\}$ is a lower semicontinuous proper convex function which is bounded from below (i.e. inf $f>-\infty)$. Changing $f$ to $f-\inf f$, we may assume that $\inf f=0$. There is also no loss of generality to assume that the set of minimizers argmin $f(=[f=0])$ contains 0 whenever it is nonempty.

In this convex setting, Theorem 13 can be considerably reinforced; related results are gathered in Section 4.1. We also recall well-known facts ensuring that 
subgradient curves have finite length and provide a new result in that direction (see Theorem 281). In Section 4.2, we give some conditions which ensure that $f$ satisfies the KE-inequality and we show that the conclusions of Theorem 20 can somehow be globalized. In Section 4.3 we build a counterexample of a $C^{2}$ convex function in $\mathbb{R}^{2}$ which does not satisfy the KE-inequality. This counterexample also reveals that the uniform boundedness of the lengths of subgradient curves is a strictly weaker condition than condition (iii) of Theorem 20. which gives a further justification for the study of piecewise subgradient curves.

4.1. Lengths of subgradient curves for convex functions. The following lemma gathers well-known complements to Theorem 13 when $f$ is convex.

Lemma 26. Let $f: H \rightarrow \mathbb{R} \cup\{+\infty\}$ be a lower semicontinuous convex function such that $\min f=f(0)=0$. Let $x_{0} \in \operatorname{dom} f$.

(i) If $a \in \operatorname{argmin} f$, then

$$
\frac{d}{d t}\left\|\chi_{x_{0}}(t)-a\right\|^{2} \leq-2 f\left(\chi_{x_{0}}(t)\right) \leq 0 \text { a.e. on }(0,+\infty),
$$

and therefore $t \mapsto\left\|\chi_{x_{0}}(t)-a\right\|$ is nonincreasing.

(ii) The function $t \mapsto f\left(\chi_{x_{0}}(t)\right)$ is nonincreasing and converges to 0 as $t \rightarrow+\infty$.

(iii) The function $t \in[0,+\infty) \longmapsto \| \partial f\left(\chi_{x_{0}}(t) \|_{-}\right.$is nonincreasing.

(iv) The function $t \mapsto f\left(\chi_{x_{0}}(t)\right)$ is convex and belongs to $L^{1}([0,+\infty))$; namely,

$$
\int_{0}^{T} f\left(\chi_{x_{0}}(t)\right) d t=\frac{1}{2}\left\|x_{0}\right\|^{2}-\frac{1}{2}\left\|\chi_{x_{0}}(T)\right\|^{2} \leq \frac{1}{2}\left\|x_{0}\right\|^{2}, \quad \text { for all } T>0 .
$$

(v) For all $T>0$,

$$
\int_{0}^{T}\left\|\dot{\chi}_{x_{0}}(t)\right\| d t \leq\left(\int_{0}^{+\infty} f\left(\chi_{x_{0}}(t)\right) d t\right)^{1 / 2}(\log T)^{1 / 2} .
$$

Proof. The proofs of these classical properties can be found in [11, 12].

Bruck established in 12 that subgradient trajectories of convex functions are always weakly converging to some point (minimizer) in $\operatorname{argmin} f$ whenever the latter is nonempty. However, as shown later on by Baillon [7, strong convergence does not hold in general.

To the best of our knowledge, the problem of the characterization of length boundedness of subgradient curves for convex coercive functions is still open (see [11, Open problems, p. 167]). It has been recently established in [20 that subgradient trajectories of convex coercive functions defined on $\mathbb{R}^{2}$ have uniformly bounded lengths, but the same assertion in $\mathbb{R}^{n}$ with $n>2$ is an open question. In this framework, the following result of Brézis [10, 11] is of particular interest.

Theorem 27 (Uniform boundedness of trajectory lengths [10). Let $f: H \rightarrow$ $\mathbb{R} \cup\{+\infty\}$ be a lower semicontinuous convex function such that $\min f=f(0)=0$. For any $x_{0} \in \operatorname{dom} f$, the subgradient curve $\chi_{x_{0}}(\cdot)$ has finite length, provided

$$
\operatorname{int}(\operatorname{argmin} f) \neq \emptyset \text {. }
$$

More precisely, for all $\rho>0$ and $T \geq 0$ we have

$$
\left.B(0, \rho) \subset \operatorname{argmin} f \Longrightarrow \int_{0}^{T} \| \dot{\chi}_{x_{0}}(t)\right) \| d t \leq \frac{1}{2 \rho}\left(\left\|x_{0}\right\|^{2}-\left\|\chi_{x_{0}}(T)\right\|^{2}\right) .
$$


Proof. We assume that $B(0, \rho) \subset \operatorname{argmin} f$ for some $\rho>0$ and consider $x_{0} \in$ $\operatorname{dom} f \backslash \operatorname{argmin} f$ (otherwise there is nothing to prove). Let $t \geq 0$ such that $\chi_{x_{0}}(t) \notin$ $\operatorname{argmin} f$ and $\dot{\chi}_{x_{0}}(t)$ exists. By convexity, we get

$$
\left\langle-\left(\chi_{x_{0}}(t)-\rho u\right), \dot{\chi}_{x_{0}}(t)\right\rangle \geq f\left(\chi_{x_{0}}(t)\right)-f(\rho u)>0
$$

for all $u$ in the unit sphere of $H$. As a consequence $-\left\langle\chi_{x_{0}}(t), \dot{\chi}_{x_{0}}(t)\right\rangle>\rho\left\|\dot{\chi}_{x_{0}}(t)\right\|$. Therefore $\int_{0}^{T}\left\|\dot{\chi}_{x_{0}}(t)\right\| d t \leq(2 \rho)^{-1}\left(\left\|x_{0}\right\|^{2}-\left\|\chi_{x_{0}}(T)\right\|^{2}\right)$.

The following result is an extension of Theorem 27 under the assumption that the vector subspace $\operatorname{span}(C)$ generated by $C:=\operatorname{argmin} f$ has codimension one in $H$. We denote by $\operatorname{ri}(C)$ the relative interior of $C$ in $\operatorname{span}(C)$.

Theorem 28. Let $f: H \rightarrow \mathbb{R} \cup\{+\infty\}$ be a lower semicontinuous convex function satisfying $\min f=f(0)=0$. Let $C:=\operatorname{argmin} f$, assume the subspace $\operatorname{span}(C)$ has codimension 1 and the relative interior $\operatorname{ri}(C)$ of $C$ with respect to $\operatorname{span}(C)$ is not empty. If $x_{0} \in \operatorname{dom} f$ is such that $\chi_{x_{0}}(t)$ converges (strongly) to $a \in \operatorname{ri}(C)$ as $t \rightarrow+\infty$, then length $\left[\chi_{x_{0}}\right]<+\infty$.

Proof. Let us denote by $a$ the limit point of $\chi(t):=\chi_{x_{0}}(t)$ as $t$ goes to infinity. By assumption $a$ belongs to $\operatorname{ri}(C)$, so that there exists $\delta>0$ such that $\bar{B}(a, \delta) \cap$ $\operatorname{span}(C) \subset C$. Let $T>0$ be such that $\chi(t) \in B(a, \delta)$ for all $t \geq T$. Write $\operatorname{span}(C)=\left\{x \in H:\left\langle x, x^{*}\right\rangle=0\right\}$ with $x^{*} \in H$. We claim that the function $[T,+\infty) \ni t \mapsto h(t)=\left\langle x^{*}, \chi(t)\right\rangle$ has a constant sign. Let us argue by contradiction and assume that there exist $T<t_{1}<t_{2}$ such that $h\left(t_{1}\right)<0<h\left(t_{2}\right)$. Hence there exists $t_{3} \in\left(t_{1}, t_{2}\right)$ such that $h\left(t_{3}\right)=0$. Since $\chi(t) \in B(a, \delta)$, this implies $\chi\left(t_{3}\right) \in C$ and thus by the uniqueness theorem for subgradient curves (Theorem 13), we have $\chi(t)=\chi\left(t_{3}\right)$ for all $t \geq t_{3}$, which is a contradiction. Note also that if $h\left(t_{0}\right)=0$ for some $t_{0} \geq T$, then $\chi$ has finite length. Indeed applying Theorem 13 once more, we deduce that $\chi(t)=\chi\left(t_{0}\right)$ for all $t \geq t_{0}$; hence

$$
\int_{0}^{+\infty}\|\dot{\chi}\|=\int_{0}^{t_{0}}\|\dot{\chi}\| \leq \sqrt{t_{0}} \sqrt{\int_{0}^{t_{0}}\|\dot{\chi}\|^{2}}<+\infty .
$$

Assume that $h$ is positive (the case $h$ negative can be treated similarly) and define the following function:

$$
\tilde{f}(x)= \begin{cases}0, & \text { if }\left\langle x, x^{*}\right\rangle<0 \text { and } x \in \bar{B}(a, \delta), \\ f(x), & \text { if }\left\langle x, x^{*}\right\rangle \geq 0 \text { and } x \in \bar{B}(a, \delta), \\ +\infty, & \text { otherwise. }\end{cases}
$$

One can easily check that the function $\tilde{f}$ is proper, lower semicontinuous and convex and that $\operatorname{argmin} \tilde{f}$ has nonempty interior. Note also that $\partial \tilde{f}(x)=\partial f(x)$ for all $x \in B(a, \delta)$ satisfying $\left\langle x, x^{*}\right\rangle>0$. The conclusion follows from Theorem 27 since $\dot{\chi}(t)+\partial \tilde{f}(\chi(t)) \ni 0$ a.e. on $(T,+\infty)$.

4.2. KŁ-inequality for convex functions. We now prove that for convex functions $f$, the desingularizing function $\varphi$ of Theorem 18(i) can be taken to be concave with domain $[0, \infty)$.

Theorem 29 (Subgradient inequality - convex case). Let $f: H \rightarrow \mathbb{R} \cup\{+\infty\}$ be a lower semicontinuous proper convex function with inf $f=0$. The following statements are equivalent and imply the existence of a minimizer. 
(i) There exist $r_{0}>0$ and $\varphi \in \mathcal{K}\left(0, r_{0}\right)$ such that

$$
\|\partial(\varphi \circ f)(x)\|_{-} \geq 1, \quad \text { for all } x \in\left[0<f \leq r_{0}\right] .
$$

(ii) There exists a concave function $\psi \in \mathcal{K}(0, \infty)$ such that

$$
\|\partial(\psi \circ f)(x)\|_{-} \geq 1, \quad \text { for all } x \notin[f=0] .
$$

Proof. The nonemptiness of $[f=0]$ under any of the assumptions (i) or (ii) follows from Corollary 4 , while the implication (ii) $\Longrightarrow(\mathrm{i})$ is obvious. To prove $(\mathrm{i}) \Longrightarrow(\mathrm{ii})$, let us first establish that the function

$$
r \in(0,+\infty) \longmapsto u(r)=\frac{1}{\inf _{x \in[f=r]}\|\partial f(x)\|_{-}}
$$

is finite-valued and nonincreasing. Let $0<r_{2}<r_{1}$ and let us show that $u\left(r_{2}\right) \geq$ $u\left(r_{1}\right)$. To this end we may assume with no loss of generality that $u\left(r_{1}\right)>0$ (and therefore that $\left[f=r_{1}\right] \cap \operatorname{dom} \partial f$ is nonempty). Take $\epsilon>0$ and let $x_{1} \in\left[f=r_{1}\right]$ and $p_{1} \in \partial f\left(x_{1}\right)$ such that $u(r) \leq \frac{1}{\left\|p_{1}\right\|}+\epsilon$. Since the continuous function $t \mapsto f\left(\chi_{x_{1}}(t)\right)$ tends to $\inf f=0$ as $t$ goes to infinity (see [41] for instance), there exists $t_{2}>0$ such that $f\left(\chi_{x_{1}}\left(t_{2}\right)\right)=r_{2}$. From Lemma 26 (iii), we obtain

$$
\frac{1}{\left\|\partial f\left(\chi_{x_{1}}\left(t_{2}\right)\right)\right\|_{-}} \geq \frac{1}{\left\|p_{1}\right\|} \geq u\left(r_{1}\right)-\epsilon
$$

which yields $u\left(r_{2}\right) \geq u\left(r_{1}\right)$. By (i) the function $u$ is finite-valued on $\left(0, r_{0}\right)$; thus, since $u$ is nonincreasing, it is also finite-valued on $(0,+\infty)$.

It is easy to see that $[(\mathrm{i}) \Rightarrow(\mathrm{v})]$ of Theorem 18 holds without the compactness assumption (24) (see Remark 19). It follows that $u \in L^{1}\left(0, r_{0}\right)$ and by Lemma 45 (Annex) that there exists a decreasing continuous function $\tilde{u} \in L^{1}\left(0, r_{0}\right)$ such that $\tilde{u} \geq u$. Reproducing the proof of $(v) \Rightarrow(i)$ of Theorem 18 we obtain a strictly increasing, concave, $C^{1}$ function

$$
\psi(r):=\int_{0}^{r} \tilde{u}(s) d s
$$

for which (39) holds for all $x \in\left[0<f<r_{0}\right]$. Fix $\bar{r} \in\left(0, r_{0}\right)$ and take $\psi$ as above. Applying (39) and using the fact that $u(r)$ is decreasing we obtain

$$
1 \leq \psi^{\prime}(\bar{r}) u(\bar{r})^{-1} \leq \psi^{\prime}(\bar{r}) u(r)^{-1} \leq \psi^{\prime}(\bar{r})\|p\|,
$$

for all $p \in \partial f(x), x \in[\bar{r} \leq f]$ and $r \in(\bar{r},+\infty)$ such that $u(r)>0$. This shows that the function $\Psi: \mathbb{R}_{+} \rightarrow \mathbb{R}_{+}$defined by

$$
\Psi(r):= \begin{cases}\psi(r), & \text { if } r \leq \bar{r} \\ \psi(\bar{r})+\psi^{\prime}(\bar{r})(r-\bar{r}), & \text { otherwise }\end{cases}
$$

satisfies the required properties.

A natural question arises: when does a convex function $f$ satisfy the KEinequality? In finite dimensions a positive answer can be given whenever $f$ belongs to an o-minimal structure (convexity then becomes superfluous). The following result gives an alternative criterion when $f$ is not extremely "flat" around its set of 
minimizers. More precisely, we assume the following growth condition with respect to a subset $S$ of $H$.

(40)

$$
\left\{\begin{array}{l}
\text { There exists } m:[0,+\infty) \rightarrow[0,+\infty) \text { and } S \subset H \text { such that } \\
m \text { is continuous, increasing, } m(0)=0, f \geq m(\operatorname{dist}(\cdot, \operatorname{argmin} f)) \text { on } S \cap \operatorname{dom} f \\
\text { and } \int_{0}^{\rho} \frac{m^{-1}(r)}{r} d r<+\infty(\text { for some } \rho>0) .
\end{array}\right.
$$

Theorem 30 (Growth assumptions and Kurdyka-Łojasiewicz inequality). Let $f$ : $H \rightarrow \mathbb{R} \cup\{+\infty\}$ be a lower semicontinuous proper convex function satisfying (40) and let us assume $f(0)=\min f$. Then the KE-inequality holds; i.e.

$$
\|\partial(\varphi \circ f)(x)\|_{-} \geq 1, \text { for all } x \in S \backslash \operatorname{argmin} f,
$$

with

$$
\varphi(r)=\int_{0}^{r} \frac{m^{-1}(s)}{s} d s .
$$

Proof. Set $C:=\operatorname{argmin} f$. Let $x \in S \cap \operatorname{dom} \partial f$ and $a$ be the projection of $x$ onto the convex subset $C$. Using the convex inequality we have

$$
\begin{aligned}
f(x)-f(a) \leq\left\langle\partial^{0} f(x), x-a\right\rangle & \leq \operatorname{dist}(0, \partial f(x)) \operatorname{dist}(x, C) \\
& \leq \operatorname{dist}(0, \partial f(x)) m^{-1}(f(x)) .
\end{aligned}
$$

Using the chain rule (see Lemma 44) and the fact that $f(a)=0$, we obtain dist $(0, \partial(\varphi \circ f)(x)) \geq 1$, where $\varphi$ is as above (note that $\varphi \in \mathcal{K}(0, \rho)$ ).

Remark 31. Assume that $H$ is finite-dimensional, and let $S$ be a compact convex subset of $H$ which satisfies $S \cap \operatorname{argmin} f \neq \emptyset$. Then there exists a convex continuous increasing function $m: \mathbb{R}_{+} \rightarrow \mathbb{R}_{+}$with $m(0)=0$ such that $f(x) \geq m(\operatorname{dist}(x, \operatorname{argmin} f))$ for all $x \in S$.

Sketch of the proof. Set $C:=\operatorname{argmin} f$. With no loss of generality we assume that $0 \in S \cap C$. Using the Moreau-Yosida regularization (see [11] for instance), we obtain the existence of a finite-valued convex continuous function $g: H \rightarrow \mathbb{R}$ such that $f \geq g$ and $\operatorname{argmin} f=\operatorname{argmin} g$. Set $\alpha=\max \{\operatorname{dist}(x, C): x \in S\}$ and $m_{0}(s)=$ $\min \{g(x): x \in S$, dist $(x, C) \geq s\} \in \mathbb{R}_{+}$for all $s \in[0, \alpha]$. Let $0 \leq s_{1}<s_{2} \leq \alpha$, and let $x_{2} \in S$ be such that dist $\left(x_{2}, C\right) \geq s_{2}$ and $0<g\left(x_{2}\right)=m\left(s_{2}\right)$. Using the convexity of $g$ and the fact that $0 \in \operatorname{argmin} g \cap S$, we see that there exists $\lambda \in(0,1)$ such that $g\left(\lambda x_{2}\right)<g\left(x_{2}\right), \lambda x_{2} \in S$ (recall that $S$ is convex and contains 0 ), and dist $\left(\lambda x_{2}, C\right) \geq s_{1}$. This shows that the function $m_{0}$ is finite-valued increasing on $[0, \alpha]$ and satisfies $m_{0}(\operatorname{dist}(x, C)) \leq g(x) \leq f(x)$ for any $x \in S$. Applying Lemma 46 (Annex) to $m_{0}$, we obtain a smooth increasing finite-valued function $m$ such that $0<m(s) \leq m_{0}(s)$ for $s \in[0, \alpha]$ with $m(0)=0$. The conclusion follows by extending $m$ to an increasing continuous function on $\mathbb{R}_{+}$.

Example 32. Take $0<\alpha<1$. If $m(r)=\exp \left(-1 / r^{\alpha}\right)$ and $m(0)=0$, then for $0 \leq s \leq \rho<1$ we have $m^{-1}(s)=1 /(-\log s)^{1 / \alpha}$ and

$$
\int_{0}^{\rho} \frac{m^{-1}(s)}{s} d s<+\infty .
$$

Therefore any convex function which is minorized by the function $x \mapsto \exp (-1 /$ $\left.\operatorname{dist}(x, C)^{\alpha}\right)$ in some neighborhood of $C=\operatorname{argmin} f$ satisfies the KL-inequality. 
4.3. A smooth convex counterexample to the $\mathbf{K} \mathbf{E}$-inequality. In this section we construct a $C^{2}$ convex function on $\mathbb{R}^{2}$ with compact level sets that fails to satisfy the KŁ-inequality. This counterexample is constructed as follows:

- we first note that any sequence of sublevel sets of a convex function that satisfies the KE-inequality must comply with a specific property ;

- we build a sequence $T_{k}$ of nested convex sets for which this property fails ;

- we show that there exists a smooth convex function which admits $T_{k}$ as sublevel sets.

The last part relies on the use of support functions and a result of Kannai [36] (see also the Ph.D. Thesis of Torralba 52]). For any closed convex subset $T$ of $\mathbb{R}^{n}$, we define its support function by $\sigma_{T}\left(x^{*}\right)=\sup _{x \in T}\left\langle x, x^{*}\right\rangle$ for all $x^{*} \in \mathbb{R}^{n}$. Let $f: \mathbb{R}^{n} \rightarrow \mathbb{R}$ be a convex function and $x^{*} \in \mathbb{R}^{n}$. Fenchel observed that the function $\lambda \mapsto \sigma_{[f \leq \lambda]}\left(x^{*}\right)$ is concave and nondecreasing ([27]). The following result asserts that this fact somehow provides a sufficient condition to rebuild a convex function starting from a countable family of nested convex sets. (We denote by int $S$ the interior of $S$ in $\mathbb{R}^{n}$.)

Theorem 33 (Convex functions with prescribed level sets [36, 52]). Let $\left\{T_{k}\right\}_{k \in \mathbb{N}}$ be a sequence of convex compact subsets of $\mathbb{R}^{n}$ such that

$$
T_{k+1} \subset \operatorname{int} T_{k} \quad \text { for all } k \geq 0 .
$$

For every $k \in \mathbb{N}$ set

$$
K_{k}=\max _{\left\|x^{*}\right\|=1} \frac{\sigma_{T_{k-1}}\left(x^{*}\right)-\sigma_{T_{k}}\left(x^{*}\right)}{\sigma_{T_{k}}\left(x^{*}\right)-\sigma_{T_{k+1}}\left(x^{*}\right)} \in(0,+\infty) .
$$

Then for every decreasing sequence $\left\{\lambda_{k}\right\}_{k \in \mathbb{N}}$ satisfying $\lambda_{0}>0$ and

$$
0<K_{k}\left(\lambda_{k}-\lambda_{k+1}\right) \leq \lambda_{k-1}-\lambda_{k}, \text { for each } k>0,
$$

there exists a continuous convex function $f$ such that

$$
T_{k}=\left[f \leq \lambda_{k}\right], \quad \text { for every } k \in \mathbb{N} .
$$

Remark 34. (i) If $\left\{\lambda_{k}\right\}$ is as in the above theorem and $x^{*} \in \mathbb{R}^{n} \backslash\{0\}$, we have

$$
\lambda_{k}-\lambda_{k+1} \leq \frac{\lambda_{0}-\lambda_{1}}{\sigma_{T_{0}}\left(x^{*}\right)-\sigma_{T_{1}}\left(x^{*}\right)}\left(\sigma_{T_{k}}\left(x^{*}\right)-\sigma_{T_{k+1}}\left(x^{*}\right)\right) \text {. }
$$

Since the sum $\sum\left(\sigma_{T_{k}}\left(x^{*}\right)-\sigma_{T_{k+1}}\left(x^{*}\right)\right)$ converges, so does the sum $\sum\left(\lambda_{k}-\lambda_{k+1}\right)$, yielding the existence of the $\operatorname{limit} \lim \lambda_{k}$. Since $f$ is the greatest function admitting $\left\{T_{k}\right\}$ as prescribed sublevel sets, we obtain $\min f=\lim \lambda_{k}$. (ii) Let $k \geq 0$ and $\lambda \in\left[\lambda_{k+1}, \lambda_{k}\right]$. The function $f$ further satisfies

$$
[f \leq \lambda]=\left(\frac{\lambda-\lambda_{k+1}}{\lambda_{k}-\lambda_{k+1}}\right) T_{k}+\left(\frac{\lambda_{k}-\lambda}{\lambda_{k}-\lambda_{k+1}}\right) T_{k+1} ;
$$

see [52, Remark 5.9].

The following lemma provides a decreasing sequence of convex compact subsets in $\mathbb{R}^{2}$ which cannot be a sequence of prescribed sublevel sets of a function satisfying the KŁ-inequality (see the last part (Conclusion) of the proof of Theorem 36).

Lemma 35. There exists a decreasing sequence of convex compact subsets $\left\{T_{k}\right\}_{k}$ of $\mathbb{R}^{2}$ such that:

(i) $T_{0}$ is the unit disk $D:=B(0,1)$;

(ii) $T_{k+1} \subset$ int $T_{k}$ for every $k \in \mathbb{N}$; 
(iii) $\bigcap_{k \in \mathbb{N}} T_{k}$ is the disk $D_{r}:=B(0, r)$ for some $r>0$;

(iv) $\sum_{k=0}^{+\infty} \operatorname{Dist}\left(T_{k}, T_{k+1}\right)=+\infty$.

Proof. We proceed by constructing the boundaries $\partial T_{k}$ of $T_{k}$ for each positive integer $k$. Let $C_{2,3}$ denote the circle of radius 1 and let us define recursively a sequence of closed convex curves $C_{n, m}$ for $n \geq 3$ and $1 \leq m \leq n+1$. For each $n \geq 3$, let $C_{n-1, n}$ be the circle of radius $R_{n}>0$. Let $\left\{\mu_{n}\right\}_{n}$ be a sequence in $(0,1)$ (which will be defined later in order to comply with property $(i i i)$ ). Then, for $1 \leq m \leq n$, let us define $C_{n, m}$ to be the union of the segments:

- $\left[\mu_{n}^{m} R_{n} \exp \left(2 \mathrm{i} \pi\left(\frac{j}{n}\right)\right), \mu_{n}^{m} R_{n} \exp \left(2 \mathrm{i} \pi\left(\frac{j+1}{n}\right)\right)\right]$ for $0 \leq j \leq m-1$ (here $i$ stands for the imaginary unit) and the circle-arc:

- $\mu_{n}^{m} R_{n} \exp (\mathrm{i} \theta)$ for $2 \pi \frac{m}{n} \leq \theta \leq 2 \pi$.

In other words, $C_{n, m}$ consists of the first $m$ edges of a regular convex $n$-polygon inscribed in a circle of radius $\mu_{n}^{m} R_{n}$ and a circle-arc of the same radius to close the curve. We then set

$$
R_{n+1}=\mu_{n}^{n+1} R_{n} \cos \left(\frac{\pi}{n}\right)
$$

and define $C_{n, n+1}$ to be the circle of radius $R_{n+1}$. Figure 1 illustrates the curves $C_{4,5}$ and $C_{5, m}$ for $m=1, \ldots, 6$.

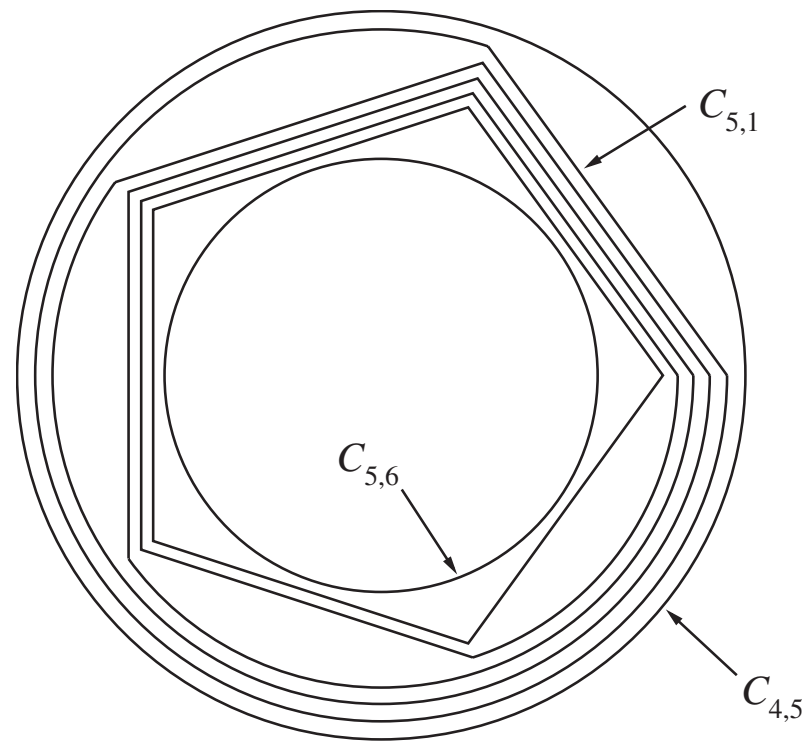

Figure 1. The curves $C_{4,5}, C_{5,1}$ to $C_{5,6}$

Ordering $\{(n, m): n \geq 3,1 \leq m \leq n+1\}$ lexicographically we define successively the convex subsets $T_{k}$ as the convex envelopes of the sets $C_{n, m}$. By construction $(i)$ and (ii) are satisfied. Item (iii) holds if $\lim R_{n}>0$, which is equivalent to the fact that the infinite product $\Pi_{n=3}^{+\infty} \mu_{n}^{n+1} \cos (\pi / n)$ does not converge to 0 . This can be achieved by taking $\mu_{n}=1-1 / n^{3}$. Let $r>0$ be the limit of $\left\{R_{n}\right\}$. The intersection of the convex sets $T_{n}$ is the disk of radius $r$. 
Take $n \geq 3$. Considering the middle of the segment $\left[\mu_{n} R_{n}, \mu_{n} R_{n} \exp \left(\frac{2 \mathrm{i} \pi}{n}\right)\right]$ in $C_{n, 1}$ and the point $R_{n} \exp \left(\frac{\mathrm{i} \pi}{n}\right) \in C_{n-1, n}$, we obtain $\operatorname{Dist}\left(C_{n, 1}, C_{n-1, n}\right)=$ $R_{n}\left(1-\mu_{n} \cos (\pi / n)\right)$. If $2 \leq m \leq n$, considering the middle of

$$
\left[\mu_{n}^{m} R_{n} \exp \left(\frac{2 \mathrm{i} \pi(m-1)}{n}, \mu_{n}^{m} R_{n} \exp \left(\frac{2 \mathrm{i} \pi m}{n}\right)\right]\right.
$$

in $C_{n, m}$ and the point $\mu_{n}^{m-1} R_{n} \exp \left(\frac{\mathrm{i} \pi(2 m-1)}{n}\right) \in C_{n, m-1}$, we get $\operatorname{Dist}\left(C_{n, m}, C_{n, m-1}\right)$ $=\mu_{n}^{m-1} R_{n}\left(1-\mu_{n} \cos (\pi / n)\right)$. Finally considering the points $\mu_{n}^{n} R_{n} \in C_{n, n}$ and $\mu_{n}^{n+1} \cos (\pi / n) R_{n} \in C_{n, n+1}$, we obtain

$$
\operatorname{Dist}\left(C_{n, n}, C_{n, n+1}\right)=\mu_{n}^{n} R_{n}\left(1-\mu_{n} \cos (\pi / n)\right) .
$$

Thus

$$
\begin{array}{r}
\operatorname{Dist}\left(C_{n, 1}, C_{n-1, n}\right)+\sum_{m=2}^{n+1} \operatorname{Dist}\left(C_{n, m}, C_{n, m-1}\right)=\sum_{m=1}^{n+1} \mu_{n}^{m-1} R_{n}\left(1-\mu_{n} \cos \frac{\pi}{n}\right) \\
\sim n r \frac{\pi^{2}}{2 n^{2}}=\frac{\pi^{2} r}{2 n} .
\end{array}
$$

Hence $(i v)$ holds.

For $\theta \in \mathbb{R} / 2 \pi \mathbb{Z}$, set $n(\theta)=(\cos \theta, \sin \theta)$ and $\tau(\theta)=(-\sin \theta, \cos \theta)$. We say that a closed $C^{2}$ curve $C$ in $\mathbb{R}^{2}$ is convex if its curvature has constant sign. If moreover the curvature never vanishes, then there exists a $C^{1}$ parametrization $c: \mathbb{R} / 2 \pi \mathbb{Z} \rightarrow C$ of $C$, called parametrization of $C$ by its normal, such that the unit tangent vector at $c(\theta)$ is $\tau(\theta)$. In this case $n(\theta)$ is the outward normal to the convex envelope of $C$ at $c(\theta)$. Moreover, $c$ is $C^{\infty}$, whenever $C$ is so. In this case, we denote by $\rho_{c}(\theta)$ the curvature radius of $c$ at $c(\theta)$ and we have

$$
\dot{c}(\theta)=\rho_{c}(\theta) \tau(\theta) .
$$

Let us denote by $T$ the convex envelope of $C$. Using the fact that $n$ defines the outward normals to $T$, we get

$$
\langle c(\theta), n(\theta)\rangle=\max _{x \in T}\langle x, n(\theta)\rangle=\sigma_{T}(n(\theta)), \forall \theta \in \mathbb{R} / 2 \pi \mathbb{Z} .
$$

Theorem 36 (Convex counterexample). There exists a $C^{2}$ convex function $f$ : $\mathbb{R}^{2} \rightarrow \mathbb{R}$ with $\min f=0$ which does not satisfy the KE-inequality and whose set of minimizers is compact with nonempty interior. More precisely, for each $r>0$ and for each desingularization function $\varphi \in \mathcal{K}(0, r)$ we have

$$
\inf \{\|\nabla(\varphi \circ f)(x)\|: x \in[0<f<r]\}=0 .
$$

Remark 37. (i) It can be seen from the forthcoming proof that $\operatorname{argmin} f$ is the closed disk centered at 0 of radius $r$, and that $f$ is actually $C^{\infty}$ on the complement of the circle of radius $r$.

(ii) The fact that $f$ is $C^{2}$ shows that the KE-inequality is not related to the smoothness of $f$. Besides, it seems clear from the proof that a $C^{k}$ ( $k$ arbitrary) counterexample could be obtained.

(iii) Since $\operatorname{argmin} f$ has nonempty interior, Theorem 27 shows that the lengths of subgradient curves are uniformly bounded. Using the notation and the results of Theorem 20. we see that the counterexample also shows that the uniform boundedness of the lengths of the subgradient curves (starting from a prescribed level set 
$\left.\left[f=r_{0}\right]\right)$ does not imply the uniform boundedness of the lengths of the piecewise subgradient curves $\gamma$ lying in $\left[\min f<f<r_{0}\right]$.

Proof of Theorem 36. Let $M, N$ be two topological finite-dimensional manifolds. In this proof, a mapping $F: M \rightarrow N$ is said to be proper if for each compact subset $K$ of $N, F^{-1}(K)$ is a compact subset of $M$. Given two nonempty subsets $A$ and $B$ of a metric space $X$ we denote by $\operatorname{Dist}(A, B)$ their Hausdorff distance (see (7D) and we define

$$
\mathrm{D}(A, B):=\inf \{d(a, b): a \in A, b \in B\} .
$$

The forthcoming proof is constructive. We start by considering a sequence of convex compact sets $\left\{T_{k}\right\}$ of $\mathbb{R}^{2}$ having the properties described in Lemma 35. Such a sequence cannot belong to the family of the sublevel sets of a smooth function $f$, unless the boundaries of $T_{k}$ are smooth manifolds. In the next step, we smoothen this sequence, conserving however its characteristic properties.

Smoothing the sequence $T_{k}$. Set $C_{k}=\partial T_{k}$ and consider a positive sequence $\epsilon_{k}$ such that $\sum \epsilon_{k}<+\infty$ with $\epsilon_{k}+\epsilon_{k+1}<\mathrm{D}\left(C_{k}, C_{k+1}\right)$ for each integer $k$. The $\epsilon_{k}$-neighborhood of $C_{k}$ can be seen to be disjoint from the $\epsilon_{k^{\prime}}$-neighborhood of $C_{k^{\prime}}$ whenever $k \neq k^{\prime}$. We can deform $C_{k}$ into a $C^{\infty}$ convex closed curve $\widetilde{C}_{k}$ whose curvature never vanishes, lying in the $\epsilon_{k}$-neighborhood of $C_{k}$. This smooth deformation can be achieved by letting $C_{k}$ evolve under the mean-curvature flow during a very short time; see [26] for the smoothing aspects and [29, 54] for the positive curvature results. Let $\widetilde{T}_{k}$ be the closed convex envelope of $\widetilde{C}_{k}$. This process provides a decreasing sequence of compact convex sets $\left\{\widetilde{T}_{k}\right\}$ that satisfies the conditions of Lemma 35. Note that the circle of radius 1 has nonzero curvature and we set $C_{0}=\widetilde{C}_{0}$. Since $\operatorname{Dist}\left(\widetilde{T}_{k}, \widetilde{T}_{k+1}\right) \geq \operatorname{Dist}\left(T_{k}, T_{k+1}\right)-\left(\epsilon_{k}+\epsilon_{k+1}\right)$ and $\sum \epsilon_{k}<+\infty$, condition (iv) holds. With no loss of generality we may therefore assume that for each $k \geq 0$ the curve $\partial T_{k}$ is smooth and can be parameterized by its normal.

Let $K_{k}$ be as in Theorem 33 and let $\lambda_{0}$ and $\lambda_{1}$ be such that $\lambda_{0}>\lambda_{1}$. We define $\lambda_{k}$ recursively by

$$
K_{k}\left(\lambda_{k}-\lambda_{k+1}\right)=\frac{1}{2}\left(\lambda_{k-1}-\lambda_{k}\right) .
$$

Thanks to (43), Theorem 33 provides a continuous convex function $f: T_{0} \rightarrow \mathbb{R}$ such that $T_{k}=\left[f \leq \lambda_{k}\right]$. Since $f$ is the greatest function with this property, we deduce that $\min f=\lim \lambda_{k}$ and $\operatorname{argmin} f=\bigcap_{k \in \mathbb{N}} T_{k}$.

Smoothness of the function $f$ on $\mathbb{R}^{2} \backslash\left(\bigcup_{k} C_{k}\right)$. We can extend $f$ outside $T_{0}$ into a smooth convex function. Let us examine the restriction of $f$ to $T_{0}$. Since $C_{k}=\partial T_{k}$ can be parameterized by its normal, we denote by $c_{k}: \mathbb{R} / 2 \pi \mathbb{Z} \rightarrow \mathbb{R}^{2}$ this parametrization. Fix $k$ in $\mathbb{N}$ and take $\theta$ in $\mathbb{R} / 2 \pi \mathbb{Z}$. Using Remark 34 (ii), we obtain

$$
\begin{aligned}
\max _{x \in[f \leq \lambda]}\langle x, n(\theta)\rangle & =\left(\frac{\lambda-\lambda_{k+1}}{\lambda_{k}-\lambda_{k+1}}\right) \max _{x \in T_{k}}\langle x, n(\theta)\rangle+\left(\frac{\lambda_{k}-\lambda}{\lambda_{k}-\lambda_{k+1}}\right) \max _{x \in T_{k+1}}\langle x, n(\theta)\rangle \\
& =\left(\frac{\lambda-\lambda_{k+1}}{\lambda_{k}-\lambda_{k+1}}\right)\left\langle c_{k}(\theta), n(\theta)\right\rangle+\left(\frac{\lambda_{k}-\lambda}{\lambda_{k}-\lambda_{k+1}}\right)\left\langle c_{k+1}(\theta), n(\theta)\right\rangle \\
& =\left\langle\left(\frac{\lambda-\lambda_{k+1}}{\lambda_{k}-\lambda_{k+1}}\right) c_{k}(\theta)+\left(\frac{\lambda_{k}-\lambda}{\lambda_{k}-\lambda_{k+1}}\right) c_{k+1}(\theta), n(\theta)\right\rangle .
\end{aligned}
$$


Using (41]) once more, we obtain

$$
\left(\frac{\lambda-\lambda_{k+1}}{\lambda_{k}-\lambda_{k+1}}\right) c_{k}(\theta)+\left(\frac{\lambda_{k}-\lambda}{\lambda_{k}-\lambda_{k+1}}\right) c_{k+1}(\theta) \in[f \leq \lambda] .
$$

Since the above maximum is attained in $[f=\lambda]$, it follows that

$$
f\left(\left(\frac{\lambda-\lambda_{k+1}}{\lambda_{k}-\lambda_{k+1}}\right) c_{k}(\theta)+\left(\frac{\lambda_{k}-\lambda}{\lambda_{k}-\lambda_{k+1}}\right) c_{k+1}(\theta)\right)=\lambda .
$$

Let us define $G: \mathbb{R} \times \mathbb{R} / 2 \pi \mathbb{Z} \rightarrow \mathbb{R}^{2}$ by

$$
G(\lambda, \theta)=\left(\frac{\lambda-\lambda_{k+1}}{\lambda_{k}-\lambda_{k+1}}\right) c_{k}(\theta)+\left(\frac{\lambda_{k}-\lambda}{\lambda_{k}-\lambda_{k+1}}\right) c_{k+1}(\theta) .
$$

The map $G$ is clearly $C^{\infty}$. Since $\frac{\partial G}{\partial \lambda}=\frac{c_{k}(\theta)-c_{k+1}(\theta)}{\lambda_{k}-\lambda_{k+1}}$, we have

$$
\begin{aligned}
\left\langle\frac{\partial G}{\partial \lambda}, n(\theta)\right\rangle & =\left\langle\frac{c_{k}(\theta)-c_{k+1}(\theta)}{\lambda_{k}-\lambda_{k+1}}, n(\theta)\right\rangle=\frac{\left\langle c_{k}(\theta), n(\theta)\right\rangle-\left\langle c_{k+1}(\theta), n(\theta)\right\rangle}{\lambda_{k}-\lambda_{k+1}} \\
& =\frac{\max _{x \in T_{k}}\langle x, n(\theta)\rangle-\max _{x \in T_{k+1}}\langle x, n(\theta)\rangle}{\lambda_{k}-\lambda_{k+1}}>0 .
\end{aligned}
$$

On the other hand

$$
\frac{\partial G}{\partial \theta}=\left(\left(\frac{\lambda-\lambda_{k+1}}{\lambda_{k}-\lambda_{k+1}}\right) \rho_{c_{k}}(\theta)+\left(\frac{\lambda_{k}-\lambda}{\lambda_{k}-\lambda_{k+1}}\right) \rho_{c_{k+1}}(\theta)\right) \tau(\theta) .
$$

Since $\rho_{c_{k}}>0$ and $\rho_{c_{k+1}}>0, G$ is a local diffeomorphism on $\left(\lambda_{k+1}-\delta, \lambda_{k}+\delta\right) \times$ $\mathbb{R} / 2 \pi \mathbb{Z}$ for any $\delta>0$ sufficiently small. In view of (44), we have $G(\lambda, \theta) \in\left[\lambda_{k+1} \leq\right.$ $\left.f \leq \lambda_{k}\right]$ for $\lambda_{k+1} \leq \lambda \leq \lambda_{k}$ and $G(\lambda, \theta) \in\left[\lambda_{k+1}<f<\lambda_{k}\right]$ for $\lambda_{k+1}<\lambda<\lambda_{k}$. Since the map $\widetilde{G}:\left[\lambda_{k+1}, \lambda_{k}\right] \times \mathbb{R} / 2 \pi \mathbb{Z} \rightarrow\left[\lambda_{k+1} \leq f \leq \lambda_{k}\right]$ defined by $\widetilde{G}(\lambda, \theta)=G(\lambda, \theta)$ is proper, $\widetilde{G}$ is a covering map from $\left[\lambda_{k+1}, \lambda_{k}\right] \times \mathbb{R} / 2 \pi \mathbb{Z}$ to $\left[\lambda_{k+1} \leq f \leq \lambda_{k}\right]$. The set $\left[\lambda_{k+1} \leq f \leq \lambda_{k}\right]$ is connected; thus $\widetilde{G}$ is onto. Using (44) and $G\left(\lambda_{k}, \theta\right)=c_{k}(\theta)$, one sees that $\left(\lambda_{k}, \theta\right)$ is the only antecedent of $c_{k}(\theta)$ by $\widetilde{G}$ and, since $\left[\lambda_{k+1}, \lambda_{k}\right] \times \mathbb{R} / 2 \pi \mathbb{Z}$ is connected, $\widetilde{G}$ is injective. Thus $\widetilde{G}$ is a $C^{\infty}$ diffeomorphism (see [40, Proposition 2.19]). By (44), this implies that the restriction of $f$ to $\left[\lambda_{k+1} \leq f \leq \lambda_{k}\right]$ is $C^{\infty}$. Using (44), we know that the level line $[f=\lambda]$ (for each $\lambda_{k+1} \leq \lambda \leq \lambda_{k}$ ) is parameterized by $G(\lambda, \theta)$ for $\theta \in \mathbb{R} / 2 \pi \mathbb{Z}$; if $c_{\lambda}$ denotes this parametrization, then $c_{k}=c_{\lambda_{k}}$. Besides, by (46), $c_{\lambda}$ is a parametrization by the normal and $\rho_{c_{\lambda}}$ is a convex combination of $\rho_{c_{k}}$ and $\rho_{c_{k+1}}$; hence $\rho_{c_{\lambda}}>0$.

Let us compute $\nabla f$ at $c_{\lambda}(\theta)$. Equation (44) yields $1=\left\langle\nabla f(G(\lambda, \theta)), \frac{\partial G}{\partial \lambda}(\lambda, \theta)\right\rangle$. Besides, we also know that the normal to $[f=\lambda]$ at $c_{\lambda}(\theta)$ is $n(\theta)$. Since the gradient $\nabla f(G(\lambda, \theta))$ and the normal $n(\theta)$ are linearly dependent, we obtain

$$
\nabla f\left(c_{\lambda}(\theta)\right)=\frac{\lambda_{k}-\lambda_{k+1}}{\left\langle c_{\lambda_{k}}(\theta)-c_{\lambda_{k+1}}(\theta), n(\theta)\right\rangle} n(\theta) .
$$

Note that this expression does not depend on $\lambda \in\left[\lambda_{k+1}-\lambda_{k}\right]$.

(Smoothing $f$ around $\left[f=\lambda_{k}\right]$.) We have seen that the function $f$ is $C^{\infty}$ on the complement of the union of the level lines $\left[f=\lambda_{k}\right]$ for $k \in \mathbb{N}$. In order to go further we need to modify $f$ around each $\left[f=\lambda_{k}\right]$. To this end, let us observe/recall two facts. 
- First using the aforementioned result of Fenchel [27, we deduce from the convexity of $f$ that the function

$$
\lambda \mapsto\left\langle c_{\lambda}(\theta), n(\theta)\right\rangle=\sigma_{[f \leq \lambda]}(n(\theta)) \text { is concave and increasing. }
$$

- Let $\lambda$ and $\lambda^{\prime}$ be such that $\lambda_{k+1} \leq \lambda \leq \lambda^{\prime} \leq \lambda_{k}$. We have

$$
\begin{aligned}
c_{\lambda}(\theta) & =\left(\frac{\lambda-\lambda_{k+1}}{\lambda^{\prime}-\lambda_{k+1}}\right) c_{\lambda^{\prime}}(\theta)+\left(\frac{\lambda^{\prime}-\lambda}{\lambda^{\prime}-\lambda_{k+1}}\right) c_{\lambda_{k+1}}(\theta), \\
c_{\lambda^{\prime}}(\theta) & =\left(\frac{\lambda^{\prime}-\lambda}{\lambda_{k}-\lambda}\right) c_{\lambda_{k}}(\theta)+\left(\frac{\lambda_{k}-\lambda^{\prime}}{\lambda_{k}-\lambda}\right) c_{\lambda}(\theta) .
\end{aligned}
$$

Consider now a positive sequence $\left\{\epsilon_{k}\right\}$ such that $\sum_{i} \epsilon_{i}<+\infty$ and $\epsilon_{k}+\epsilon_{k+1}<$ $\mathrm{D}\left(C_{k}, C_{k+1}\right)=\mathrm{D}\left(\left[f=\lambda_{k}\right],\left[f=\lambda_{k+1}\right]\right)$ for each integer $k$. Let us assume that there exists a sequence

$$
f_{k}: \mathbb{R}^{2} \rightarrow \mathbb{R}
$$

of convex functions such that:

(P1) $f_{0}=f$;

(P2) $f_{k}=f_{k-1}$ outside an $\epsilon_{k}$-neighborhood of $\left[f=\lambda_{k}\right]$;

(P3) $f_{k}$ is $C^{\infty}$ on $\left[f>\lambda_{k+1}\right]$;

(P4) $\left\|\nabla f_{k}\right\|$ is bounded in $\left[f \leq \lambda_{k}\right]$ by the maximum of $\|\nabla f\|$ in $\left[\lambda_{k} \leq f \leq \lambda_{k-1}\right]$.

Let us choose $k \geq 1$ and $\lambda, \lambda^{\prime}$ such that $\lambda_{k+1} \leq \lambda \leq \lambda_{k} \leq \lambda^{\prime} \leq \lambda_{k-1}$. Then by (43) and (47) we have:

$$
\begin{aligned}
\left\|\nabla f\left(c_{\lambda}(\theta)\right)\right\| & =\frac{\lambda_{k}-\lambda_{k+1}}{\left\langle c_{\lambda_{k}}(\theta)-c_{\lambda_{k+1}}(\theta), n(\theta)\right\rangle} \\
& \leq \frac{1}{2} \frac{\lambda_{k-1}-\lambda_{k}}{\left\langle c_{\lambda_{k-1}}(\theta)-c_{\lambda_{k}}(\theta), n(\theta)\right\rangle}=\frac{1}{2} \| \nabla f\left(c_{\lambda^{\prime}}(\theta) \| .\right.
\end{aligned}
$$

Hence

$$
\max _{\left[\lambda_{k+1} \leq f \leq \lambda_{k}\right]}\|\nabla f\| \leq \frac{1}{2} \max _{\left[\lambda_{k} \leq f \leq \lambda_{k-1}\right]}\|\nabla f\| .
$$

Combining with (P4), the above implies that the sequence $\left\{f_{k}\right\}_{k \in \mathbb{N}}$ is uniformly Lipschitz continuous. Applying the Ascoli compactness theorem, we obtain that the $f_{k}$ converge to a continuous function $\tilde{f}$ which is convex. From (P2) and (P3), we obtain successively that $\tilde{f}$ has the same set of minimizers as $f, f$ is $C^{\infty}$ outside $\operatorname{argmin} \tilde{f},\left[\tilde{f}=\lambda_{k}\right]$ is in the $\epsilon_{k}$-neighborhood of $\left[f=\lambda_{k}\right]$. Moreover (51) and (P4) imply that $\|\nabla \tilde{f}(x)\|$ goes to zero as $x$ approaches $\operatorname{argmin} \tilde{f}$; hence $\tilde{f}$ is globally $C^{1}$. Note also that the sequence of level sets $\left[\tilde{f} \leq \lambda_{k}\right]$ satisfies the hypothesis $(i v)$ of Lemma 35. As shown in the conclusion, $\tilde{f}$ provides a $C^{1}$ counterexample to the KŁ-inequality.

Let us define such a sequence $\left\{f_{k}\right\}$ by induction. Assume that $f_{k-1}$ is defined. In order to construct $f_{k}$, it suffices to proceed in the $\epsilon_{k}$-neighborhood of $\left[f=\lambda_{k}\right]$. Let $\epsilon>0$ such that $\left[\lambda_{k}-2 \epsilon \leq f \leq \lambda_{k}+2 \epsilon\right]$ is in the $\epsilon_{k}$-neighborhood of $\left[f=\lambda_{k}\right]$. Let us consider a $C^{\infty}$ function $\mu_{-}:[-2 \epsilon, 2 \epsilon] \rightarrow \mathbb{R}$ which satisfies the following properties:
1. $\mu_{-}$is nonincreasing,
2. $\mu_{-}^{\prime \prime} \geq 0$,
3. $\mu_{-}(\lambda)=-\lambda / \epsilon$ on $[-2 \epsilon,-\epsilon / 2]$,
4. $\mu_{-}(\lambda)=0$ on $[\epsilon / 2,2 \epsilon]$. 
Let us then define $\mu_{+}(\lambda):=\lambda / \epsilon+\mu_{-}(\lambda)$ and $\mu_{0}=1-\left(\mu_{-}+\mu_{+}\right)$. The function $\mu_{+}$satisfies

$$
\begin{array}{ll}
1^{\prime} \cdot \mu_{+} \text {is nondecreasing, } & 2^{\prime} \cdot \mu_{+}^{\prime \prime}=\mu_{-}^{\prime \prime} \geq 0, \\
3^{\prime} \cdot \mu_{+}(\lambda)=0 \text { on }[-2 \epsilon,-\epsilon / 2], & 4^{\prime} \cdot \mu_{+}(\lambda)=\lambda / \epsilon \text { on }[\epsilon / 2,2 \epsilon] .
\end{array}
$$

Set $c_{-}=c_{\lambda_{k}-\epsilon}, c_{0}=c_{\lambda_{k}}, c_{+}=c_{\lambda_{k}+\epsilon}$ and

$$
\begin{aligned}
& M_{-}(\theta)=\left\langle c_{-}(\theta), n(\theta)\right\rangle=\max _{x \in\left[f \leq \lambda_{k}-\epsilon\right]}\langle x, n(\theta)\rangle, \\
& M_{0}(\theta)=\left\langle c_{0}(\theta), n(\theta)\right\rangle=\max _{x \in\left[f \leq \lambda_{k}\right]}\langle x, n(\theta)\rangle, \\
& M_{+}(\theta)=\left\langle c_{+}(\theta), n(\theta)\right\rangle=\max _{x \in\left[f \leq \lambda_{k}+\epsilon\right]}\langle x, n(\theta)\rangle .
\end{aligned}
$$

For $(\lambda, \theta) \in[-2 \epsilon, 2 \epsilon] \times \mathbb{R} / 2 \pi \mathbb{Z}$, we define:

$$
H(\lambda, \theta)=\mu_{-}(\lambda) c_{-}(\theta)+\mu_{0}(\lambda) c_{0}(\theta)+\mu_{+}(\lambda) c_{+}(\theta) .
$$

Then $H$ is a $C^{\infty}$ map and for any $\lambda \in[-\epsilon, \epsilon]$, the real numbers $\mu_{-}(\lambda), \mu_{0}(\lambda)$ and $\mu_{+}(\lambda)$ are in $[0,1]$. Since $H(\lambda, \theta)$ is a convex combination of points in $\left[f \leq \lambda_{k}+\epsilon\right]$, we deduce that $H(\lambda, \theta) \in\left[f \leq \lambda_{k}+\epsilon\right]$ and $H(\lambda, \theta) \in\left[f<\lambda_{k}+\epsilon\right]$ whenever $\lambda<\epsilon$ and $\mu_{+}(\lambda)<1$. Since

$$
\langle H(\lambda, \theta), n(\theta)\rangle=\mu_{-}(\lambda) M_{-}(\theta)+\mu_{0}(\lambda) M_{0}(\theta)+\mu_{+}(\lambda) M_{+}(\theta) \geq M_{-}(\theta),
$$

we get $H(\lambda, \theta) \in\left[f \geq \lambda_{k}-\epsilon\right]$, and $H(\lambda, \theta) \in\left[f>\lambda_{k}-\epsilon\right]$ whenever $\lambda>\epsilon, \mu_{-}(\lambda)<1$. It follows that

$$
\frac{\partial H}{\partial \lambda}=\mu_{-}^{\prime}(\lambda) c_{-}(\theta)+\mu_{0}^{\prime}(\lambda) c_{0}(\theta)+\mu_{+}^{\prime}(\lambda) c_{+}(\theta) .
$$

Since $\mu_{0}^{\prime}=-\mu_{-}^{\prime}-\mu_{+}^{\prime}$, items 1 and $1^{\prime}$ entail

$$
\begin{aligned}
\left\langle\frac{\partial H}{\partial \lambda}, n(\theta)\right\rangle & =\mu_{+}^{\prime}(\lambda)\left\langle c_{+}(\theta)-c_{0}(\theta), n(\theta)\right\rangle-\mu_{-}^{\prime}(\lambda)\left\langle c_{0}(\theta)-c_{-}(\theta), n(\theta)\right\rangle \\
& =\mu_{+}^{\prime}(\lambda)\left(M_{+}(\theta)-M_{0}(\theta)\right)-\mu_{-}^{\prime}(\lambda)\left(M_{0}(\theta)-M_{-}(\theta)\right) \\
& >0 .
\end{aligned}
$$

On the other hand

$$
\frac{\partial H}{\partial \theta}=\left(\mu_{-}(\lambda) \rho_{c_{-}}(\theta)+\mu_{0}(\lambda) \rho_{c_{0}}(\theta)+\mu_{+}(\lambda) \rho_{c_{+}}(\theta)\right) \tau(\theta),
$$

so that $\left\langle\frac{\partial H}{\partial \theta}, n(\theta)\right\rangle=0$ and $\left\langle\frac{\partial H}{\partial \theta}, \tau(\theta)\right\rangle>0$ for $\left.\lambda \epsilon\right]-\epsilon^{\prime}, \epsilon^{\prime}\left[\right.$ with $\epsilon^{\prime}>\epsilon$. Thus $H$ is a local diffeomorphism on $]-\epsilon^{\prime}, \epsilon^{\prime}\left[\times \mathbb{R} / 2 \pi \mathbb{Z}\right.$. The map $\widetilde{H}:[-\epsilon, \epsilon] \times \mathbb{R} / 2 \pi \mathbb{Z} \rightarrow\left[\lambda_{k}-\epsilon \leq\right.$ $\left.f \leq \lambda_{k}+\epsilon\right]$ defined by $\widetilde{H}(\lambda, \theta)=H(\lambda, \theta)$ is proper; therefore $\widetilde{H}$ is a covering map from $[-\epsilon, \epsilon] \times \mathbb{R} / 2 \pi \mathbb{Z}$ to $\left[\lambda_{k}-\epsilon \leq f \leq \lambda_{k}+\epsilon\right]$. Since $\left[\lambda_{k}-\epsilon \leq f \leq \lambda_{k}+\epsilon\right]$ is connected, $\widetilde{H}$ is onto. Besides, since $c_{+}(\theta) \in\left[f=\lambda_{+} \epsilon\right],(\epsilon, \theta)$ is the only antecedent of $c_{+}(\theta)$ by $H, \widetilde{H}$ is injective by connectedness of $[-\epsilon, \epsilon] \times \mathbb{R} / 2 \pi \mathbb{Z}$. $\widetilde{H}$ is therefore a $C^{\infty}$ diffeomorphism from $[-\epsilon, \epsilon] \times \mathbb{R} / 2 \pi \mathbb{Z}$ into $\left[\lambda_{k}-\epsilon \leq f \leq \lambda_{k}+\epsilon\right]$.

We then define $f_{k}$ to be $f_{k-1}$ outside of $\left[\lambda_{k}-\epsilon \leq f \leq \lambda_{k}+\epsilon\right]$ and by $f_{k}(H(\lambda, \theta))=$ $\lambda_{k}+\lambda$ in $\left[\lambda_{k}-\epsilon \leq f \leq \lambda_{k}+\epsilon\right]$. When $\lambda \in\left[\lambda_{k}-\epsilon, \lambda_{k}-\epsilon / 2\right]$, Properties $3,3^{\prime}$ and 
equation (49) yield

$$
\begin{aligned}
H\left(\lambda-\lambda_{k}, \theta\right) & =-\frac{\lambda-\lambda_{k}}{\epsilon} c_{-}(\theta)+\left(1+\frac{\lambda-\lambda_{k}}{\epsilon}\right) c_{0}(\theta) \\
& =\frac{\lambda_{k}-\lambda}{\lambda_{k}-\left(\lambda_{k}-\epsilon\right)} c_{-}(\theta)+\frac{\lambda-(\lambda-\epsilon)}{\lambda_{k}-\left(\lambda_{k}-\epsilon\right)} c_{0}(\theta) \\
& =c_{\lambda}(\theta) .
\end{aligned}
$$

Thus $f_{k}=f=f_{k-1}$ in $\left[\lambda_{k}-\epsilon \leq f \leq \lambda_{k}-\epsilon / 2\right]$ and, for similar reasons, $f_{k}=f_{k-1}$ in $\left[\lambda_{k}+\epsilon / 2 \leq f \leq \lambda_{k}+\epsilon\right]$. The "gluing" of $f_{k-1}$ and $f_{k}$ is therefore $C^{\infty}$ along $\left[f=\lambda_{k}-\epsilon\right]$ and $\left[f=\lambda_{k}+\epsilon\right]$. Hence, $f_{k}$ satisfies (P3).

Let us compute $\nabla f_{k}$ in $\left[\lambda_{k}-\epsilon \leq f \leq \lambda_{k}+\epsilon\right]$. By definition of $f_{k}, 1=$ $\left\langle\nabla f_{k}(H(\lambda, \theta)), \frac{\partial H}{\partial \lambda}\right\rangle$. Besides $H\left(\lambda-\lambda_{k}, \theta\right)$ is a parametrization of the level line $\left[f_{k}=\lambda\right]$ by its normal (see (52) $)$; hence $\nabla f_{k}(H(\lambda, \theta))=\alpha n(\theta)$ with $\alpha>0$. Using both formulae, we finally get

$$
\nabla f_{k}(H(\lambda, \theta))=\frac{1}{\mu_{+}^{\prime}(\lambda)\left\langle c_{+}(\theta)-c_{0}(\theta), n(\theta)\right\rangle-\mu_{-}^{\prime}(\lambda)\left\langle c_{0}(\theta)-c_{-}(\theta), n(\theta)\right\rangle} n(\theta) .
$$

From the definition of $\mu_{+}, \mu_{+}^{\prime}(\lambda)-\mu_{-}^{\prime}(\lambda)=1 / \epsilon$. Besides, for $\lambda \in[-\epsilon,-\epsilon / 2]$ we have

$$
\frac{\epsilon}{\left\langle c_{0}(\theta)-c_{-}(\theta), n(\theta)\right\rangle}=\left\|\nabla f\left(c_{\lambda+\lambda_{k}}(\theta)\right)\right\|,
$$

while for $\lambda \in[\epsilon / 2, \epsilon]$ we get

$$
\frac{\epsilon}{\left\langle c_{+}(\theta)-c_{0}(\theta), n(\theta)\right\rangle}=\left\|\nabla f\left(c_{\lambda+\lambda_{k}}(\theta)\right)\right\| .
$$

Hence by (48):

$$
\left\|\nabla f_{k}(H(\lambda, \theta))\right\| \leq\left\|\nabla f\left(c_{\lambda_{k}+\epsilon}(\theta)\right)\right\| .
$$

Item (P4) is therefore satisfied.

The last assertion we need to establish is the convexity of $f_{k}$. By construction, it suffices to prove that the Hessian $Q_{f_{k}}$ of $f$ is nonnegative in $\left[\lambda_{k}-\epsilon \leq f \leq \lambda_{k}+\epsilon\right]$. Let us denote by $Q_{H}$ the Hessian of $H$ (observe that $Q_{H}$ takes its values in $\mathbb{R}^{2}$ ). For $-\epsilon \leq \lambda \leq \epsilon$, we have $\lambda+\lambda_{k}=f_{k}(H(\lambda, \theta))$; thus

$$
0=Q_{f_{k}}(H(\lambda, \theta))(D H(\lambda, \theta)(\cdot), D H(\lambda, \theta)(\cdot))+\left\langle\nabla f_{k}(H(\lambda, \theta)), Q_{H}(\lambda, \theta)(\cdot, \cdot)\right\rangle,
$$

where $D H$ denotes the differential map of $H$. To prove that $Q_{f_{k}}$ is nonnegative, it suffices to prove that $\left\langle\nabla f_{k}(H(\lambda, \theta)), Q_{H}(\lambda, \theta)(\cdot, \cdot)\right\rangle \leq 0$. We have

$$
\begin{aligned}
\frac{\partial^{2} H}{\partial \lambda^{2}} & =\mu_{-}^{\prime \prime}(\lambda) c_{-}(\theta)+\mu_{0}^{\prime \prime}(\lambda) c_{0}(\theta)+\mu_{+}^{\prime \prime}(\lambda) c_{+}(\theta) \\
& =\mu_{-}^{\prime \prime}(\lambda)\left(c_{-}(\theta)-c_{0}(\theta)\right)+\mu_{+}^{\prime \prime}(\lambda)\left(c_{+}(\theta)-c_{0}(\theta)\right) \\
& =\mu_{+}^{\prime \prime}(\lambda)\left[\left(c_{+}(\theta)-c_{0}(\theta)\right)-\left(c_{0}(\theta)-c_{-}(\theta)\right)\right]
\end{aligned}
$$

where the last equality is due to item $2^{\prime}$. On the other hand

$$
\begin{aligned}
\left\langle\nabla f_{k}(H(\lambda, \theta)), \frac{\partial^{2} H}{\partial \lambda^{2}}\right\rangle=\mu_{+}^{\prime \prime}(\lambda) \| \nabla f_{k}\left(H(\lambda, \theta) \|\left(\left\langlec_{+}(\theta)\right.\right.\right. & \left.-c_{0}(\theta), n(\theta)\right\rangle \\
& \left.-\left\langle c_{0}(\theta)-c_{-}(\theta), n(\theta)\right\rangle\right),
\end{aligned}
$$

which is nonpositive because of (48). Besides, we have

$$
\frac{\partial^{2} H}{\partial \lambda \partial \theta}=\left(\mu_{-}^{\prime}(\lambda) \rho_{c_{-}}(\lambda)+\mu_{0}^{\prime}(\lambda) \rho_{c_{0}}(\lambda)+\mu_{+}^{\prime}(\lambda) \rho_{c_{+}}(\lambda)\right) \tau(\theta) ;
$$


thus $\left\langle\nabla f_{k}(H(\lambda, \theta)), \frac{\partial^{2} H}{\partial \lambda \partial \theta}\right\rangle=0$. Finally

$$
\frac{\partial^{2} H}{\partial \theta^{2}}=\left(\mu_{-}(\lambda) \rho_{c_{-}}(\theta)+\mu_{0}(\lambda) \rho_{c_{0}}(\theta)+\mu_{+}(\lambda) \rho_{c_{+}}(\theta)\right)(-n(\theta))+(\cdots) \tau(\theta)
$$

hence the quantity

$\left\langle\nabla f_{k}(H(\lambda, \theta)), \frac{\partial^{2} H}{\partial \theta^{2}}\right\rangle=-\left(\mu_{-}(\lambda) \rho_{c_{-}}(\theta)+\mu_{0}(\lambda) \rho_{c_{0}}(\theta)+\mu_{+}(\lambda) \rho_{c_{+}}(\theta)\right)\left\|\nabla f_{k}(H(\lambda, \theta))\right\|$

is negative since all the $\mu$ and $\rho$ are nonnegative. Hence $Q_{f_{k}}$ is nonnegative and the function $f_{k}$ is convex.

$C^{2}$ smoothing. For $\lambda \in\left(\min \tilde{f}, \lambda_{0}\right]$, define

$$
h(\lambda)=(\lambda-\min \tilde{f})\left(1+\max _{\left[\lambda \leq \tilde{f} \leq \lambda_{0}\right]}\left\|Q_{\tilde{f}}\right\|\right)^{-1} .
$$

Since $\tilde{f}$ is $C^{\infty}$ in $[\min \tilde{f}<\tilde{f}], h$ is a continuous, positive, increasing function. Then there exists $\psi \in C^{\infty}\left(\mathbb{R}, \mathbb{R}_{+}\right)$which vanishes on $(-\infty, \min \tilde{f}]$, increases on $(0,+\infty)$ and for $\lambda \in\left(\min \tilde{f}, \lambda_{0}\right], 0<\psi(\lambda) \leq h(\lambda)$ (see Lemma 46). Let $g$ be the primitive of $\psi$ with $g(\min \tilde{f})=0$. The function $g$ is a strictly increasing convex $C^{\infty}$ function on $[\min \tilde{f},+\infty)$. The function $\bar{f}=g \circ \tilde{f}$ is therefore a $C^{1}$ convex function. Moreover $\bar{f}$ is $C^{\infty}$ at each point outside the boundary of $\operatorname{argmin} f$. For $x \in \operatorname{argmin} f$, we have

$$
\begin{aligned}
\frac{\nabla \bar{f}(x+h)-\nabla \bar{f}(x)}{\|h\|} & =\frac{g^{\prime}(\tilde{f}(x+h)) \nabla \tilde{f}(x+h)}{\|h\|} \\
& =\frac{g^{\prime}(\tilde{f}(x)+o(\|h\|)) o(1)}{\|h\|}=\frac{o(\|h\|)}{\|h\|}=o(1) .
\end{aligned}
$$

Thus $Q_{\bar{f}}(x)=0$. On the other hand

$$
\begin{aligned}
\left\|Q_{\bar{f}}(x+h)\right\| & \leq g^{\prime}(\tilde{f}(x+h))\left\|Q_{\tilde{f}}(x+h)\right\|+g^{\prime \prime}(\tilde{f}(x+h))\|\nabla \tilde{f}(x+h)\|^{2} \\
& \leq h(\tilde{f}(x+h))\left\|Q_{\tilde{f}}(x+h)\right\|+o(1) \\
& \leq(f(x+h)-f(x))+o(1)=o(1) .
\end{aligned}
$$

Thus $Q_{\bar{f}}$ is continuous at $x$ and thus $\bar{f}$ is $C^{2}$.

Conclusion. Let us prove finally that $\bar{f}$ does not satisfy the KE-inequality. Towards a contradiction, let us assume that there exist $R>\inf \bar{f}=\min \bar{f}$, a continuous function $\varphi:[\min \bar{f}, R) \rightarrow \mathbb{R}_{+}$which satisfies $\varphi(\min \bar{f})=0, \varphi$ is $C^{1}$ on $(\min \bar{f}, R)$ with $\varphi^{\prime}>0$, such that we have

$$
\|\nabla(\varphi \circ \bar{f})(x)\| \geq 1, \forall x \in[\min f<f<R] .
$$

Applying Theorem 20] [(i) $\Leftrightarrow($ vi)], we obtain

$$
\operatorname{Dist}\left(\left[\bar{f} \leq g\left(\lambda_{k}\right)\right],\left[\bar{f} \leq g\left(\lambda_{k+1}\right)\right]\right) \leq \varphi\left(g\left(\lambda_{k}\right)\right)-\varphi\left(g\left(\lambda_{k+1}\right)\right),
$$

and, as a consequence,

$$
\sum_{k=0}^{+\infty} \operatorname{Dist}\left(\left[\tilde{f} \leq \lambda_{k}\right],\left[\tilde{f} \leq \lambda_{k+1}\right]\right)=\sum_{k=0}^{+\infty} \operatorname{Dist}\left(\left[\bar{f} \leq g\left(\lambda_{k}\right)\right],\left[\bar{f} \leq g\left(\lambda_{k+1}\right)\right]\right) \leq \varphi\left(g\left(\lambda_{0}\right)\right) .
$$

This contradicts the fact that $\sum \operatorname{Dist}\left(T_{k}, T_{k+1}\right)=+\infty$.

A careful glance of the previous proof reveals in particular the following result of independent interest. (Recall (42) for the definition of the distance D.) 
Proposition 38 (Construction of a $C^{2}$ convex function with almost prescribed level sets). Let $\left\{\epsilon_{k}\right\}_{k \in \mathbb{N}} \subset(0,+\infty)$ and $\left\{T_{k}\right\}_{k \in \mathbb{N}}$ be a sequence of convex compact sets in $\mathbb{R}^{2}$ such that

$$
T_{k+1} \subset \operatorname{int} T_{k} \quad \text { and } \quad \mathrm{D}\left(\partial T_{k}, \partial T_{k+1}\right)>\epsilon_{k}+\epsilon_{k+1}, \quad \text { for all } k \in \mathbb{N} .
$$

Then there exists a strictly decreasing sequence $\left\{\lambda_{k}\right\}_{k \in \mathbb{N}}$ converging to 0 and a $C^{2}$ convex function $f: \mathbb{R}^{2} \rightarrow[0,+\infty)$ such that for all $k \in \mathbb{N}$,

$$
\left[f=\lambda_{k}\right] \subset \partial T_{k}+B\left(0, \epsilon_{k}\right) \quad \text { and } \quad[f=0]=\bigcap_{k \in \mathbb{N}} T_{k} .
$$

Moreover, $f$ is $C^{\infty}$ on $\mathbb{R}^{2} \backslash \operatorname{argmin} f$.

4.4. Asymptotic equivalence for discrete and continuous dynamics. In this part we assume that $f: H \rightarrow \mathbb{R}$ is a $C^{1,1}$ convex function, that is, continuously differentiable with gradient $\nabla f$ Lipschitz continuous. Let $L$ be a Lipschitz constant of $\nabla f$.

Fix $\beta>0$ and $x \in \mathbb{R}^{n}$ and consider any sequence $\left\{Y_{x}^{k}\right\}$ satisfying

$$
\left\{\begin{array}{l}
\beta\left\|\nabla f\left(Y_{x}^{k}\right)\right\|\left\|Y_{x}^{k+1}-Y_{x}^{k}\right\| \leq f\left(Y_{x}^{k}\right)-f\left(Y_{x}^{k+1}\right), \quad k=1,2, \ldots \\
Y_{x}^{0}=x
\end{array}\right.
$$

This condition has been considered in [1] for nonconvex functions defined in finitedimensional spaces. It is easily seen that (53) is a descent sequence, that is, $f\left(Y_{x}^{k}\right) \geq$ $f\left(Y_{x}^{k+1}\right)$, which implies in particular that $\left\{f\left(Y_{x}^{k}\right)\right\}$ converges as $k$ goes to infinity.

Condition (53) is fulfilled by several explicit gradient-like methods, including trust region methods, line-search gradient methods and some Riemannian variants; see [1] for examples and references.

The following theorem establishes connections between length boundedness properties of continuous gradient methods and length boundedness of discrete gradient iterations. It would be interesting to compare the forthcoming result with the results of the Ph.D. Thesis of Peypouquet [50, Chapter 8].

Theorem 39 (Discrete vs. continuous). Let $f$ be a $C^{1,1}$ convex function with compact sublevel sets such that $\min f=0$. Let us denote by L a Lipschitz constant of $\nabla f$. Then the following statements are equivalent:

(i) [Kurdyka-Eojasiewicz inequality] There exist $r_{0}>0$ and $\varphi \in \mathcal{K}\left(0, r_{0}\right)$ such that

$$
\|\nabla(\varphi \circ f)(x)\| \geq 1, \quad \text { for all } x \in\left[0<f \leq r_{0}\right] .
$$

(ii) $[$ Length boundedness of piecewise gradient iterates $]$ For all $\beta>0$ and all $R>0$, there exists $\mathcal{L}(\beta)>0$ such that for any sequence of gradient iterates of the form

$$
Y_{x_{0}}^{0}, Y_{x_{0}}^{1}, \ldots, Y_{x_{0}}^{k_{0}}, Y_{x_{1}}^{0}, \ldots Y_{x_{1}}^{k_{1}}, \ldots
$$

with $f\left(x_{0}\right)<R, f\left(Y_{x_{i+1}}^{0}\right)=f\left(x_{i+1}\right) \leq f\left(Y_{x_{i}}^{k_{i}}\right)$ and $\left\{Y_{x_{i}}^{j}: j=0, \ldots, k_{i}\right\}$ satisfying (53) for all $i \in \mathbb{N}$ we have

$$
\sum_{i=0}^{+\infty} \sum_{l=0}^{k_{i}}\left\|Y_{x_{i}}^{l+1}-Y_{x_{i}}^{l}\right\| \leq \mathcal{L}(\beta)
$$


(iii) [Length boundedness of piecewise gradient curves] For every $R>0$ there exists $\mathcal{L}>0$ such that

$$
\text { length }(\gamma) \leq \mathcal{L}
$$

for all piecewise subgradient curves $\gamma:[0,+\infty) \rightarrow H$ with $f(\gamma(0))<R$.

Proof. Let us first prove that (i) $\Rightarrow$ (ii). By Theorem 29 [(i) $\Rightarrow$ (ii)] (subgradient inequality - convex case) we may assume that $\varphi$ is concave, defined on $(0,+\infty)$ and (54) holds for all $x \in[0<f]$. We now proceed in the spirit of [1. Let $\beta>0$, $x \in[0<f]$ and let $Y_{x}^{0}, \ldots, Y_{x}^{k}$ be a (finite) sequence of gradient-type iterations that satisfy (53). For simplicity set $Y_{x}^{j}=Y^{j}$ for all $j \in\{0, \ldots, k\}$, so that

$$
f\left(Y^{j}\right)-f\left(Y^{j+1}\right) \geq \beta\left\|\nabla f\left(Y^{j}\right)\right\|\left\|Y^{j+1}-Y^{j}\right\| \text {. }
$$

Multiplying both parts with $\varphi^{\prime}\left(f\left(Y^{j}\right)\right)$ and applying (i) we get

$$
\varphi^{\prime}\left(f\left(Y^{j}\right)\right)\left[f\left(Y^{j}\right)-f\left(Y^{j+1}\right)\right] \geq \beta\left\|Y^{j+1}-Y^{j}\right\| .
$$

Since $\varphi$ is concave we have

$$
\varphi\left(f\left(Y^{j+1}\right)\right) \leq \varphi\left(f\left(Y^{j}\right)\right)+\varphi^{\prime}\left(f\left(Y^{j}\right)\right)\left[f\left(Y^{j+1}\right)-f\left(Y^{j}\right)\right]
$$

and therefore

$$
\varphi\left(f\left(Y^{j}\right)\right)-\varphi\left(f\left(Y^{j+1}\right)\right) \geq \beta\left\|Y^{j+1}-Y^{j}\right\| .
$$

Adding the above inequalities for $j=0, \ldots, k$ we obtain

$$
\varphi\left(f\left(Y^{0}\right)\right)-\varphi\left(f\left(Y^{k}\right)\right) \geq \beta \sum_{j=0}^{k}\left\|Y^{j+1}-Y^{j}\right\| .
$$

Let us now consider a sequence of the form $\left\{Y_{x_{0}}^{0}, Y_{x_{0}}^{1}, \ldots, Y_{x_{0}}^{k_{0}}, Y_{x_{1}}^{0}, \ldots Y_{x_{1}}^{k_{1}}, \ldots\right\}$ as in (ii). Then applying (55) to each subsequence $\left\{Y_{x_{i}}^{j}, j=0, \ldots, k_{i}\right\}$ we deduce

$$
\sum_{i=0}^{+\infty} \sum_{l=0}^{k_{i}}\left\|Y_{x_{i}}^{l+1}-Y_{x_{i}}^{l}\right\|<\frac{1}{\beta} \varphi\left(f\left(Y_{x_{0}}^{0}\right)\right) \leq \frac{1}{\beta} \varphi(R),
$$

which proves the assertion.

The equivalence (i) $\Longleftrightarrow$ (iii) follows from Theorem 18 and Theorem 29 To complete the proof it suffices to establish that (ii) implies the assertion (iv) of Theorem 18 (existence of a talweg of finite length). (In fact we prove (iv') with $R=2$; see Remark 19 (i).) So let us assume that (ii) holds and let $r_{0}>m$. We aim to construct a piecewise absolutely continuous curve $\theta:\left(0, r_{0}\right] \rightarrow \mathbb{R}^{n}$ of finite length that satisfies

$$
\theta(r) \in \mathcal{V}_{2}(r):=\left\{x \in[f=r]: \quad\|\nabla f(x)\| \leq 2 \inf _{y \in[f=r]}\|\nabla f(y)\|\right\}, \forall r \in\left(0, r_{0}\right] .
$$

We shall use the explicit gradient method described in Subsection 5.2. Let $x_{0} \in$ $\mathcal{V}_{2}\left(r_{0}\right)$ be such that

$$
\left\|\nabla f\left(x_{0}\right)\right\| \leq \frac{3}{2} \inf _{y \in f^{-1}\left(r_{0}\right)}\|\nabla f(y)\|
$$

and consider the $C^{1}$ curve

$$
\left[0, \frac{1}{3 L}\right) \ni t \longmapsto x_{0}(t):=x_{0}-t \nabla f\left(x_{0}\right) .
$$

Set $t_{0}=\sup A_{0}$, where

$$
A_{0}:=\left\{t \in\left(0, \frac{1}{3 L}\right):\left[\begin{array}{cr}
f \circ x_{0} & \text { strictly decreasing on }[0, t], \\
x_{0}(\tau) \in \mathcal{V}_{2}\left(f\left(x_{0}(\tau)\right)\right. & \text { for } \tau \in[0, t]
\end{array}\right\}\right.
$$


Clearly $A_{0}$ is nonempty and $0<t_{0} \leq(3 L)^{-1}$. Set $r_{1}=f\left(x_{0}\left(t_{0}\right)\right)<r_{0}$ and take $x_{1} \in \mathcal{V}_{2}\left(r_{1}\right)$ such that

$$
\left\|\nabla f\left(x_{1}\right)\right\| \leq \frac{3}{2} \inf _{y \in\left[f=r_{1}\right]}\|\nabla f(y)\| .
$$

Proceeding by induction we obtain a sequence $\left\{\left(t_{k}, r_{k}, x_{k}\right)\right\}$, where $\left\{r_{k}\right\} \subset\left[0, r_{0}\right]$ is decreasing, $x_{n}(t):=x_{n}-t \nabla f\left(x_{n}\right)$ with $f\left(x_{n}\right)=r_{n}$ and

$$
\left\|\nabla f\left(x_{n}\right)\right\| \leq \frac{3}{2} \inf _{y \in\left[f=r_{n}\right]}\|\nabla f(y)\| .
$$

Let us denote by $r_{\infty}$ the limit of $\left\{r_{k}\right\}$ and let us assume, towards a contradiction, that $r_{\infty}>0$. Set

$$
s(r):=\inf _{x \in f^{-1}(r)}\|\partial f(x)\|_{-} \quad \text { and } \quad s_{\infty}=\liminf _{n \rightarrow \infty} s\left(r_{n}\right)=\lim _{n \rightarrow \infty} s\left(r_{n}\right)
$$

(note that the convexity of $f$ guarantees that $s\left(r_{1}\right) \leq s\left(r_{2}\right)$ whenever $r_{1} \leq r_{2}$ ) and observe that $r_{\infty}>0$ implies that $s_{\infty}>0$ (use the compactness of the sublevel set $\left.\left[f \leq r_{0}\right]\right)$. Let $n_{0} \in \mathbb{N}$ be such that $s\left(r_{n}\right) \leq \frac{5}{4} s_{\infty}$ for all $n \geq n_{0}$. For $n \geq n_{0}$ and $t \in\left[0, t_{n}\right.$ ), Proposition 49 (Annex) yields

$$
\left\|\nabla f\left(x_{n}(t)\right)\right\| \leq(L t+1)\left\|\nabla f\left(x_{n}\right)\right\|,
$$

which implies

$$
\left\|\nabla f\left(x_{n}(t)\right)\right\| \leq(L t+1)\left\|\nabla f\left(x_{n}\right)\right\| \leq \frac{3}{2}(L t+1) s\left(r_{n}\right) \leq \frac{15}{8}(L t+1) s_{\infty} .
$$

A sufficient condition to have $x_{n}(t) \in \mathcal{V}_{2}\left(f\left(x_{n}(t)\right)\right)$ is therefore

$$
\frac{15}{8}(L t+1) s_{\infty} \leq 2 s_{\infty} \Longleftrightarrow 0 \leq t \leq(15 L)^{-1} .
$$

Similarly we can estimate the rate of decrease of $f\left(x_{n}(t)\right)$. Since

$$
\frac{d}{d t} f\left(x_{n}(t)\right)=-\left\langle\nabla f\left(x_{n}\right), \nabla f\left(x_{n}(t)\right)\right\rangle,
$$

the condition $\frac{d}{d t} f\left(x_{n}(t)\right)<0$ is satisfied whenever

$$
\left\|\nabla f\left(x_{n}\right)\right\|^{2}>\left\|\nabla f\left(x_{n}\right)\right\|\|\| f\left(x_{n}(t)\right)-\nabla f\left(x_{n}\right) \| .
$$

But since $\nabla f$ is Lipschitz continuous, $\left\|\nabla f\left(x_{n}(t)\right)-\nabla f\left(x_{n}\right)\right\| \leq L t\left\|\nabla f\left(x_{n}\right)\right\|$. Thus the condition is satisfied if

$$
\left\|\nabla f\left(x_{n}\right)\right\|^{2}>L t\left\|\nabla f\left(x_{n}\right)\right\|^{2} .
$$

This last inequality is equivalent to $t<L^{-1}$, which implies in particular that for all $n \in \mathbb{N}$ such that $s\left(r_{n}\right) \leq \frac{5}{4} s_{\infty}$, we have

$$
t_{n} \geq(15 L)^{-1} \text {. }
$$

In this case Proposition 49(Annex) yields

$$
\begin{aligned}
f\left(x_{n}\left(t_{n}\right)\right) \leq f\left(x_{n}\right)+\left(\frac{L t_{n}^{2}}{2}-t_{n}\right)\left\|\nabla f\left(x_{n}\right)\right\|^{2} & \leq r_{n}+\frac{9}{4}\left(\frac{L t_{n}^{2}}{2}-t_{n}\right) s\left(r_{n}\right)^{2} \\
& \leq r_{n}+\frac{9}{4}\left(\frac{L t_{n}^{2}}{2}-t_{n}\right)\left(\frac{5}{4} s_{\infty}\right)^{2} .
\end{aligned}
$$

Thus in order to have $f\left(x_{n}(t)\right)<r_{\infty}$, it suffices to require

$$
t_{n}-\frac{L t_{n}^{2}}{2}>\frac{64}{225}\left(\frac{r_{n}-r_{\infty}^{2}}{s_{\infty}}\right) .
$$


Using the fact that $(3 L)^{-1} \geq t_{n} \geq(15 L)^{-1}$, we see that

$$
t_{n}-\frac{L t_{n}^{2}}{2} \geq(15 L)^{-1}-(18 L)^{-1}=(90 L)^{-1} .
$$

Since $\left(r_{\infty}-r_{n}\right) / s_{\infty}$ tends to zero, we have that $f\left(x_{n}\left(t_{n}\right)\right)<r_{\infty}$ for $n$ sufficiently large, which is a contradiction.

We thus conclude that $\left\{r_{k}\right\} \rightarrow r_{\infty}=0$ and $\left(0, r_{0}\right]=\bigcup_{n}\left(r_{n+1}, r_{n}\right]$. We define $\theta:\left(0, r_{0}\right] \rightarrow H$ as follows: $\theta(r):=x_{n}\left(\left[f \circ x_{n}\right]^{-1}(r)\right)$ whenever $r \in\left(r_{n+1}, r_{n}\right]$. Clearly $\theta$ defines a piecewise absolutely continuous curve. To see that $\theta$ has finite length it suffices to observe that the sequence $\left\{x_{n}\right\}_{n}$ is a sequence of gradient iterates that satisfies (53). Using Remark 50] and the fact that the step-sizes in the construction of the $x_{n}$ 's do not exceed $(3 L)^{-1}$ we infer that

$$
\frac{5}{6}\left\|x_{n+1}-x_{n}\right\|\left\|\nabla f\left(x_{n}\right)\right\| \leq f\left(x_{n}\right)-f\left(x_{n+1}\right) .
$$

Hence the curve $\theta$ has a finite length. This completes the proof.

Remark 40. The assumption that $f$ is convex has been used to apply Theorem 29 ( $c f$. concavity of $\varphi$, which seems crucial for the proof of the implication (i) $\Rightarrow($ ii)) and to assert $f\left(Y_{0}^{k}\right) \rightarrow \inf f$. For this reason we have been unable to state Theorem[39] in a more general context (i.e. for general semiconvex functions in a local version). It would therefore be interesting to figure out under which type of conditions (other than convexity or o-minimality of $f$ ) the function $\varphi$ of (54) can be taken to be concave.

\section{AnNex}

This section contains several technical results which are needed in the text.

\subsection{Technical results.}

Proposition 41 (Closed graph of the subdifferential). Let $f: H \rightarrow \mathbb{R} \cup\{+\infty\}$ be a lower semicontinuous semiconvex function. Let $\left\{x_{k}\right\}$ and $\left\{p_{k}\right\}$ be two sequences in $H$ such that $p_{k} \in \partial f\left(x_{k}\right), x_{k}$ converges strongly to $x$ and $p_{k}$ converges weakly to p. Then as $k \rightarrow+\infty$ we obtain

$$
\left\{\begin{array}{l}
f\left(x_{k}\right) \rightarrow f(x) \\
p \in \partial f(x)
\end{array}\right.
$$

Proof. This is a standard property. For a proof (in the more general setting of primer-lower-nice functions) we refer the reader to [44].

Proposition 42 (Slope functions and semicontinuity). Let $f: H \rightarrow \mathbb{R} \cup\{+\infty\}$ be a lower semicontinuous semiconvex function.

(i) The extended-real-valued function

(slope at $x$ )

$$
H \ni x \longmapsto\|\partial f(x)\|_{-}:=\inf _{p \in \partial f(x)}\|p\|
$$

is lower semicontinuous.

(ii) Take $r_{0} \in \mathbb{R}$ and let $D$ be a nonempty compact subset of $\left[f \leq r_{0}\right]$. Then the function

(minimal slope of the $r$ level-line)

$$
\left(-\infty, r_{0}\right] \ni r \longmapsto s_{D}(r):=\inf _{x \in[f=r] \cap D}\|\partial f(x)\|_{-}
$$

is lower semicontinuous. 
(iii) Assume that (23) and (24) hold for some $\bar{r}, \bar{\epsilon}>0$. If $0<r_{1} \leq r_{2} \leq \bar{r}$, then there exists $\eta_{r_{1}, r_{2}}>0$ such that

$$
\inf _{x \in\left[r_{1} \leq f \leq r_{2}\right] \cap \bar{B}(\bar{r}, \bar{\epsilon})}\|\partial f(x)\|_{-} \geq \eta_{r_{1}, r_{2}}>0 .
$$

Proof. (ii) Take $r \in\left(-\infty, r_{0}\right]$ and let $\left\{r_{k}\right\} \subset\left(-\infty, r_{0}\right]$ be a sequence such that $r_{k} \rightarrow r$ and $\liminf _{k} s_{D}\left(r_{k}\right)<+\infty$. Fix $\eta>0$ and let $\left(x_{k}, p_{k}\right) \in$ graph $\partial f$ be such that $f\left(x_{k}\right)=r_{k}, p_{k} \in \partial f\left(x_{k}\right)$ and $\left\|p_{k}\right\|<s_{D}\left(r_{k}\right)-\eta$. Using a standard compactness argument together with the fact that $\liminf _{k} s_{D}\left(r_{k}\right)<+\infty$ we can assume, with no loss of generality, that $x_{k}$ converges (strongly) to $x \in D$ and that $p_{k}$ converges weakly to $p$. Using Proposition 41, we obtain that $(x, p) \in$ graph $\partial f$ and $f(x)=r$. The conclusion follows from the (weak) lower semicontinuity of the norm. Indeed

$$
\liminf _{k \rightarrow+\infty} s_{D}\left(r_{k}\right)-\eta \geq \liminf _{k \rightarrow+\infty}\left\|p_{k}\right\| \geq\|p\| \geq s_{D}(r) .
$$

The proofs of (i) and (iii) involve similar arguments.

Lemma 43 (Strong slope). Let $f$ be a proper lower semicontinuous semiconvex function. Then for all $x$ in $H$,

$$
\|\partial f(x)\|_{-}=|\nabla f|(x)
$$

Proof. Let $x \in H$ and $p=\partial^{0} f(x)$ be the projection of 0 on $\partial f(x)$. By (18), for any $y \in H$, we have

$$
\frac{(f(x)-f(y))^{+}}{\|y-x\|} \leq\left(-\left\langle p, \frac{y-x}{\|y-x\|}\right\rangle+\alpha\|y-x\|^{2}\right)^{+} \leq\left(\|p\|+\alpha\|y-x\|^{2}\right)^{+} .
$$

By taking the limsup as $y \rightarrow x$, we get $|\nabla f|(x) \leq\|p\|=\|\partial f(x)\|_{-}$. To prove the opposite inequality, we consider the subgradient curve $\chi_{x}$. If $x$ is a critical point of $f$, then $0=\|\partial f(x)\|_{-} \geq|\nabla f|(x)$. Otherwise, $\chi_{x}(t) \neq x$ for all $t>0$. By Theorem 13(iv), we have

$$
\frac{\left(f(x)-f\left(\chi_{x}(t)\right)\right)^{+}}{\left\|x-\chi_{x}(t)\right\|} \geq \frac{1}{\left\|x-\chi_{x}(t)\right\|} \int_{0}^{t}\left\|\partial f\left(\chi_{x}(\tau)\right)\right\|_{-}^{2} d \tau .
$$

Taking the limsup as $t \downarrow 0$ and using the continuity of the semiflow and Theorem 13(ii),(iii) we obtain the desired result.

Lemma 44 (Chain rules). Let $f: H \rightarrow \mathbb{R} \cup\{+\infty\}$ be an extended-real-valued function.

(i) Let $\varphi:(0,1) \rightarrow \mathbb{R}$ be a $C^{1}$ function. Then

$$
\partial(\varphi \circ f)(x)=\varphi^{\prime}(f(x)) \partial f(x), \text { for all } x \in[0<f<1] \text {. }
$$

(ii) Let $\gamma:(0,1) \rightarrow H$ be a $C^{1}$ curve. For all $t \in(0,1)$, we have

$$
\partial(f \circ \gamma)(t) \supset\{\langle\dot{\gamma}(t), p(t)\rangle: p(t) \in \partial f(\gamma(t))\} .
$$

Proof. For the proof, see [53] for example.

Lemma 45 (Continuous integrable majorant). Let $u:\left(0, r_{0}\right] \rightarrow \mathbb{R}_{+}$be an upper semicontinuous function such that $u \in L^{1}\left(0, r_{0}\right)$. Then there exists a continuous function $w:\left(0, r_{0}\right] \rightarrow \mathbb{R}_{+}$such that $w \geq u$ and $w \in L^{1}\left(0, r_{0}\right)$. If moreover $u$ is assumed to be nonincreasing, $w$ can be chosen to be decreasing. 
Proof. With no loss of generality we assume $r_{0}=1$. Replacing $u(\cdot)$ if necessary by the function $u(\cdot)+1$ we may also assume that $u \geq 1$. Let $a_{k}>0$ be a strictly decreasing sequence such that $a_{0}=1$ and $(0,1]=\bigcup_{k \in \mathbb{N}}\left[a_{k+1}, a_{k}\right]$. Let us assume that there exists a sequence of continuous functions $w_{k}:\left[a_{k+1}, a_{k}\right] \rightarrow \mathbb{R}$ such that $w_{k} \geq u$ on $\left[a_{k+1}, a_{k}\right]$ and $\int_{a_{k+1}}^{a_{k}} w_{k} \leq \int_{a_{k+1}}^{a_{k}} u+\frac{1}{(k+1)^{2}}$. To establish the existence of $w$, we proceed by induction on $k$. Fix $k \geq 1$ and assume that $w$ is defined on $\left[a_{k}, 1\right]$ with $w \geq u, w$ continuous and

$$
\int_{a_{k}}^{1} w \leq \int_{a_{k}}^{1} u+\sum_{i=1}^{k} \frac{2}{i^{2}}
$$

There is no loss of generality to assume $w_{k}\left(a_{k}\right) \leq w\left(a_{k}\right)$ (the case $w_{k}\left(a_{k}\right)>w\left(a_{k}\right)$ can be treated analogously). Let us define

$$
0<\epsilon_{k}=\frac{w_{k}\left(a_{k}\right)\left(a_{k}-a_{k+1}\right)}{(k+1)^{2} w\left(a_{k}\right) \max _{\left[a_{k+1}, a_{k}\right]} w_{k}}<a_{k}-a_{k+1},
$$

and let us consider the functions

defined by

$$
\lambda_{k}:\left[a_{k}-\epsilon_{k}, a_{k}\right] \rightarrow\left[1, \frac{w\left(a_{k}\right)}{w_{k}\left(a_{k}\right)}\right]
$$

$$
\lambda_{k}(r)=\frac{1}{\epsilon_{k}}\left(\left(a_{k}-r\right)+\left(r-\left(a_{k}-\epsilon_{k}\right)\right) \frac{w\left(a_{k}\right)}{w_{k}\left(a_{k}\right)}\right) .
$$

The function $w$ can now be extended to $\left[a_{k+1}, 1\right]$ by setting

$$
w(r)= \begin{cases}w_{k}(r), & \text { if } r \in\left[a_{k+1}, a_{k}-\epsilon_{k}\right), \\ \lambda_{k}(r) w_{k}(r), & \text { if } r \in\left[a_{k}-\epsilon_{k}, a_{k}\right], \\ w(r), & \text { if } r \in\left(a_{k}, 1\right] .\end{cases}
$$

It is easily seen that the function $w$ is continuous (by definition of $\lambda_{k}$ ), it satisfies $w \geq u$ on $\left[a_{k+1}, a_{k}\right]$ (thus on $\left.\left(a_{k+1}, 1\right]\right)$ and moreover

$$
\begin{aligned}
\int_{a_{k+1}}^{1} w & =\int_{a_{k+1}}^{a_{k}-\epsilon_{k}} w_{k}+\int_{a_{k}-\epsilon_{k}}^{a_{k}} \lambda_{k} w_{k}+\int_{a_{k}}^{1} w \\
& \leq \int_{a_{k+1}}^{a_{k}} u+\frac{1}{(k+1)^{2}}+\epsilon_{k} \frac{w\left(a_{k}\right)}{w_{k}\left(a_{k}\right)} \max _{\left[a_{k+1}, a_{k}\right]} w_{k}+\int_{a_{k}}^{1} u+\sum_{i=1}^{k} \frac{2}{i^{2}}, \\
& \leq \int_{a_{k+1}}^{1} u+\frac{2}{(k+1)^{2}}+\sum_{i=1}^{k} \frac{2}{i^{2}} .
\end{aligned}
$$

This proves the existence of a continuous function $w$ that satisfies the required properties.

To complete the proof it suffices to prove the existence of such a sequence $\left\{w_{k}\right\}$. To this end, fix $k \in \mathbb{N}^{*}$ and set

$$
u^{\epsilon}(r)=\sup _{\rho \in\left[a_{k+1}, a_{k}\right]}\left\{u(\rho)-\frac{\|r-\rho\|^{2}}{2 \epsilon}\right\} .
$$

It is easily seen that $u^{\epsilon}$ is continuous, $u(r) \leq u^{\epsilon}(r) \leq \max _{\rho \in\left[a_{k+1}, a_{k}\right]} u:=M_{k}<+\infty$ and $\lim _{\epsilon \rightarrow 0} u^{\epsilon}(r)=u(r)$ for all $r \in\left[a_{k+1}, a_{k}\right]$ (see [53], for example). Note that the upper semicontinuity of $u$ on the compact set $\left[a_{k+1}, a_{k}\right]$ guarantees that $M_{k}$ is finite. Applying the Lebesgue domination convergence theorem we conclude that 
$u^{\epsilon}$ converges to $u$ in the norm topology of $L^{1}\left(a_{k+1}, a_{k}\right)$. Thus there exists $\epsilon_{0}>0$ such that

$$
\int_{\left[a_{k+1}, a_{k}\right]} u^{\epsilon_{0}} \leq \int_{\left[a_{k+1}, a_{k}\right]} u+\frac{1}{(k+1)^{2}} .
$$

Thus the function $w_{k}:=u^{\epsilon_{0}}$ satisfies the requirements stated above. This completes the proof of the first part of the statement. The case where $u$ is assumed decreasing can be treated with similar (and occasionally simpler) arguments.

Lemma 46. Let $h \in C^{0}\left(\left(0, r_{0}\right], \mathbb{R}_{+}^{*}\right)$ be an increasing function. Then there exists a function $\psi \in C^{\infty}\left(\mathbb{R}, \mathbb{R}_{+}\right)$such that $\psi=0$ on $\mathbb{R}_{-}, 0<\psi(s) \leq h(s)$ for all $s \in\left(0, r_{0}\right)$, and $\psi$ is increasing on $\left(0, r_{0}\right)$.

Proof. Let us extend the definition of $h$ by 0 on $\mathbb{R}_{-}$and $h\left(r_{0}\right)$ for $s>r_{0}$. Consider $\phi \in C^{\infty}\left(\mathbb{R}, \mathbb{R}_{+}\right)$with $[0,1]$ as support and $\int_{\mathbb{R}} \phi=1$. Then we define $\psi$ by $\psi=\phi * h ;$ i.e. $\psi(s)=\int_{\mathbb{R}} \phi(t) h(s-t) d t$. It is then straightforward to verify that $\psi$ satisfies the expected properties.

Proposition 47 (Piecewise absolutely continuous selections). Let $r_{0}>0$ and $\mathcal{V}$ : $\left(0, r_{0}\right] \rightrightarrows H$ be a set-valued mapping with nonempty values. Assume that for each $r \in\left(0, r_{0}\right]$ there exists $\epsilon_{r} \in(0, r)$ and an absolutely continuous curve $\theta_{r}:\left(r-\epsilon_{r}, r\right] \rightarrow$ $H$ such that

$$
\theta_{r}(s) \in \mathcal{V}(s) \text { for all } s \text { in }\left(r-\epsilon_{r}, r\right] \text {. }
$$

Then there exist a countable partition $\left\{I_{n}\right\}_{n \in \mathbb{N}}$ of $\left(0, r_{0}\right]$ into intervals $I_{n}$ of nonempty interior and a selection $\theta:\left(0, r_{0}\right] \rightarrow \mathbb{R}^{n}$ of $\mathcal{V}$ such that $\theta$ is absolutely continuous on each $I_{n}$.

Proof. Let $\Omega$ be the set of couples $\left(\alpha: I_{\alpha} \subset\left(0, r_{0}\right] \rightarrow \mathbb{R}^{n},\left\{I_{\alpha, j}\right\}_{j \in J_{\alpha}}\right)$, where $\left\{I_{\alpha, j}\right\}_{j \in J_{\alpha}}$ is a countable partition of $I_{\alpha}$ into (disjoint) intervals $I_{\alpha, j}, j \in J_{\alpha}$ with nonempty interior such that:

(a) for each $j \in J_{\alpha}, \alpha$ is absolutely continuous on $I_{\alpha, j}$,

(b) for each $r \in I_{\alpha}, \alpha(r) \in \mathcal{V}(r)$.

We define a partial order $\preccurlyeq$ on $\Omega$ by

$\alpha_{1} \preccurlyeq \alpha_{2} \Leftrightarrow \forall j \in J_{\alpha_{1}}, \exists k \in J_{\alpha_{2}}, I_{\alpha_{1}, j} \subset I_{\alpha_{2}, k}$ and $\alpha_{1}(r)=\alpha_{2}(r)$ for all $r \in I_{\alpha_{1}}$.

Note that $(\Omega, \preccurlyeq)$ is nonempty partially ordered. Let us check that each totally ordered subset of $\Omega$ has an upper bound in $\Omega$. To this end, let

$$
\omega=\left\{\left(\alpha_{l},\left\{I_{\alpha_{l}, j}\right\}_{j \in J_{\alpha_{l}}}\right)\right\}_{l \in \mathcal{L}}
$$

be a totally ordered subset of $\Omega$. For each $r \in \bigcup_{l \in \mathcal{L}} I_{\alpha_{l}}$ define $\alpha(r)$ by

$$
\alpha(r):=\alpha_{l}(r),
$$

whenever $r \in I_{l}$, and set $I_{\alpha}=\bigcup_{l \in L} I_{\alpha_{l}}$. Since $\omega$ is totally ordered, the mapping $\alpha: I_{\alpha} \rightarrow \mathbb{R}^{n}$ is well defined and (b) is clearly satisfied. For $l \in L$ and $j \in J_{l}$, set $J_{l}:=J_{\alpha_{l}}, I_{\alpha_{l}, j}=I_{l, j}$ and $D:=\left\{(m, k): m \in L, k \in J_{m}\right\}$. For each $(l, j) \in D$, let us define

$$
M_{l, j}:=\bigcup_{(m, k) \in D, I_{l, j} \subset I_{m, k}} I_{m, k} .
$$

Observe that $I_{\alpha}=\bigcup_{(l, j) \in D} M_{l, j}$ and that each $M_{l, j}$ is an interval with nonempty interior. 
Let us prove that for all $(l, j),\left(l^{\prime}, j^{\prime}\right) \in D$, we have either $M_{l^{\prime}, j^{\prime}}=M_{l, j}$ or $M_{l^{\prime}, j^{\prime}} \cap M_{l, j}=\emptyset$. In order to establish this result, let us show beforehand that for all $(l, j),\left(l^{\prime}, j^{\prime}\right)$ in $D$ such that $I_{l, j} \cap I_{l^{\prime}, j^{\prime}} \neq \emptyset$, we have $M_{l, j}=M_{l^{\prime}, j^{\prime}}$. Indeed, since $\omega$ is totally ordered, we have for instance $I_{l^{\prime}, j^{\prime}} \subset I_{l, j}$ and so $M_{l, j} \subset M_{l^{\prime}, j^{\prime}}$. Conversely, take $(m, k) \in D$ such that $I_{m, k} \supset I_{l^{\prime}, j^{\prime}}$. Since $I_{m, k} \cap I_{l, j} \neq \emptyset$, we have either $I_{m, k} \subset I_{l, j}$ or $I_{m, k} \supset I_{l, j}$; in any case, we see ( $c f$. definition (57)) that $I_{m, k} \subset M_{l, j}$ and thus $M_{l^{\prime}, j^{\prime}} \subset M_{l, j}$.

If $M_{l, j} \cap M_{l^{\prime}, j^{\prime}} \neq \emptyset$, take $r$ in the intersection, and observe that by definition there exist $(m, k)$ and $\left(m^{\prime}, k^{\prime}\right)$ in $D$ such that $I_{m, k} \supset I_{l, j}$ with $r \in I_{m, k}$ and $I_{m^{\prime}, k^{\prime}} \supset I_{l^{\prime}, j^{\prime}}$ with $r \in I_{m^{\prime}, k^{\prime}}$. Using the previous remark, we obtain that $M_{m, k}=M_{l, j}$ and $M_{m^{\prime}, k^{\prime}}=M_{l^{\prime}, j^{\prime}}$. But since $I_{m, k} \cap I_{m^{\prime}, k^{\prime}} \neq \emptyset$, we also have $M_{m, k}=M_{m^{\prime}, k^{\prime}}$ and thus $M_{l, j}=M_{l^{\prime}, j^{\prime}}$.

Let us define an equivalence relation $\simeq$ on $D$ by

$$
(l, j) \simeq\left(l^{\prime}, j^{\prime}\right) \Leftrightarrow M_{l, j}=M_{l^{\prime}, j^{\prime}}
$$

This equivalence relation defines a partition of $D$ into equivalence classes. By the axiom of choice we can pick one and only one element in each equivalence class and this defines a nonempty subset $D^{\prime}$ of $D$. By construction we have $I_{\alpha}=$ $\bigcup_{(l, j) \in D^{\prime}} M_{l, j}$ and $M_{l, j} \cap M_{l^{\prime}, j^{\prime}}=\emptyset$ for each $(l, j) \neq\left(l^{\prime}, j^{\prime}\right)$ in $D^{\prime}$. Besides, since each $M_{l, j}$ (for $(l, j) \in D^{\prime}$ ) has a nonempty interior, we see that $D^{\prime}$ is a countable set. This shows that $\left(\alpha,\left\{M_{l, j},(l, j) \in D^{\prime}\right\}\right)$ is in $\Omega$ with in addition $\alpha \geq \alpha_{l}$ for all $l \in \mathcal{L}$

Applying Zorn's lemma to $\Omega$, we obtain the existence of a maximal element $\left(\theta: I_{\theta} \rightarrow \mathbb{R}^{n},\left\{I_{\theta, j}, j \in J_{\theta}\right\}\right)$. Arguing by contradiction, we see immediately that $I_{\theta}=\left(0, r_{0}\right]$.

5.2. Explicit gradient method. We recall the following useful result.

Lemma 48 (Descent lemma). Let $f$ be a $C^{1,1}$ function (that is, $\nabla f$ is L-Lipschitz continuous). Then

$$
f(y) \leq f(x)+\langle\nabla f(x), y-x\rangle+\frac{L}{2}\|y-x\|^{2} .
$$

Proof. Set $x(t)=x+t(y-x)$ and notice that

$f(y)-f(x)=\int_{0}^{1} \frac{d}{d t} f(x(t)) d t=\langle\nabla f(x), y-x\rangle+\int_{0}^{1}\langle\nabla f(x(t))-\nabla f(x), y-x\rangle d t$.

The assertion follows easily.

Given $x \in H$, let us consider the following recursion rule:

$$
x^{+}:=X(t, x)=x-t \nabla f(x), t>0 .
$$

Choosing a starting point $x^{0}$ in $H$, and $\lambda_{k}>0$ a sequence of step-sizes, the explicit gradient method is written

$$
x^{k+1}=X\left(\lambda_{k}, x^{k}\right) .
$$

A part of the convergence analysis of this method (and some of its variants) is based on the following elementary results.

Proposition 49. Let $f$ be a $C^{1,1}$ function, $x \in H, t \in\left[0,2 L^{-1}\right)$ and $x^{+}$be given by (58). Then

(i) $\left(1-\frac{L t}{2}\right)\left\|x^{+}-x\right\|\|\nabla f(x)\| \leq f(x)-f\left(x^{+}\right)$;

(ii) $\left\|\nabla f\left(x^{+}\right)\right\| \leq(L t+1)\|\nabla f(x)\|$. 
Proof. Assertion (i) follows directly from Lemma 48 while assertion (ii) is a consequence of the fact that $\nabla f$ is Lipschitz continuous on $[x, x(t)]$ of constant $L$.

Remark 50. Condition (53) of Section 4.4 corresponds of course to the inequality (i) above.

\section{ACKNOWLEDGEMENT}

The first two authors acknowledge support of the ANR grants ANR-05-BLAN0248-01 and ANR-08-BLAN-0294-01 (France). The second author acknowledges support of the MEC grant MTM2008-06695-C03-03/MTM (Spain). During the preparation of this work, several research visits of the co-authors have been realized, respectively to the CRM (Mathematical Research Center in Barcelona), the University Autonomous of Barcelona, the University of Paris 6 and the University of Tours. In each case the concerned author wishes to acknowledge their hosts for their hospitality. The first three authors thank G. Barles for fruitful discussions. The first author thanks J.-N. Corvellec, A. Ioffe and R. Cominetti for useful comments on the first version of the manuscript.

\section{REFERENCES}

[1] Absil, P.-A., Mahony, R. \& Andrews, B., Convergence of the iterates of descent methods for analytic cost functions. SIAM J. Optim. 16 (2005), 531-547. MR2197994 (2006j:90065)

[2] Albano, P. \& Cannarsa, P., Singularities of semiconcave functions in Banach spaces, Stochastic analysis, control, optimization and applications, Systems Control Found. Appl., 171-190, Birkhäuser Boston, 1999.

[3] Ambrosio, L., Gigli, N. \& Savaré, G. Gradient flows in metric spaces and in the space of probability measures, Lectures in Mathematics ETH Zurich. Birkhäuser Verlag, Basel, 2005. MR 2129498(2006k:49001)

[4] Aussel, D., Daniilidis, A. \& Thibault, L., Subsmooth sets: Functional characterizations and related concepts, Trans. Amer. Math. Soc. 357 (2005), 1275-1301. MR2115366 (2007b:49033)

[5] Attouch, H. \& Bolte, J. On the convergence of the proximal algorithm for nonsmooth functions involving analytic features, Math. Programming 116 (2009), 5-16. MR2421270

[6] Azé, D. \& Corvellec, J.-N., Characterizations of error bounds for lower semicontinuous functions on metric spaces, ESAIM Control Optim. Calc. Var. 10 (2004), 409-425. MR2084330 (2005e:49027)

[7] Baillon, J.-B., Un exemple concernant le comportement asymptotique de la solution du problème $d u / d t+\partial \varphi(u) \ni 0, \quad J$. Funct. Anal. 28 (1978), 369-376. MR496964 (81a:47063)

[8] Bolte, J., Daniilidis, A. \& Lewis, A.S., The Łojasiewicz inequality for nonsmooth subanalytic functions with applications to subgradient dynamical systems, SIAM J. Optim. 17 (2006), 1205-1223. MR2274510 (2007j:49020)

[9] Bolte, J., Daniilidis, A., Lewis, A. \& Shiota, M., Clarke subgradients of stratifiable functions, SIAM J. Optimization 18 (2007), 556-572. MR2338451 (2008m:49092)

[10] Brézis, H., Monotonicity methods in Hilbert spaces and some applications to nonlinear partial differential equations, Contributions to nonlinear functional analysis (Proc. Sympos., Math. Res. Center, Univ. Wisconsin, Madison, Wis., 1971), Academic Press, New York, 1971, 101-156. MR0394323 (52:15126)

[11] Brézis, H., Opérateurs maximaux monotones et semi-groupes de contractions dans les espaces de Hilbert (French), North-Holland Mathematics Studies 5, North-Holland Publishing Co., 1973. MR0348562 (50:1060)

[12] Bruck, Jr., R. E., Asymptotic convergence of nonlinear contraction semigroups in Hilbert space, J. Funct. Anal. 18 (1975), 15-26. MR0377609 (51:13780)

[13] Cannarsa, P. \& Sinestrari, C., Semiconcave functions, Hamilton-Jacobi equations, and optimal control, Progress in Nonlinear Differential Equations and their Applications, 58, Birkhäuser Boston Inc., 2004. MR2041617 (2005e:49001) 
[14] Clarke, F.H., Ledyaev, Yu., Stern, R.I. \& Wolenski, P.R., Nonsmooth Analysis and Control Theory, Graduate Texts in Mathematics 178, Springer-Verlag, New-York, 1998. MR 1488695 (99a:49001)

[15] Combettes, P. \& Pennanen, T., Proximal methods for cohypomonotone operators, SIAM J. Control Optim. 43 (2004), 731-742. MR2086182(2005f:49045)

[16] Corvellec, J.-N. \& Motreanu, V., Nonlinear error bounds for lower semicontinuous functions on metric spaces, Math. Programming 114 (2008), 291-319. MR2393044 (2009b:49041)

[17] Coste, M., An Introduction to o-minimal Geometry, RAAG Notes, 81 pages, Institut de Recherche Mathématiques de Rennes, November 1999.

[18] D'Acunto, D., Sur les courbes intégrales du champ de gradient, 72 pp., Ph.D. Thesis (Université de Savoie, 2001).

[19] D'Acunto, D. \& Kurdyka, K., Bounds for gradient trajectories and geodesic diameter of real algebraic sets, Bull. London Math. Soc. 38 (2006), 951-965. MR.2285249 (2007k:14118)

[20] Daniilidis, A., Ley, O. \& Sabourau, S., Asymptotic behaviour of self-contracted planar curves and gradient orbits of convex functions, preprint 19 pp., (UAB 31/2008).

[21] Degiovanni, M., Marino, A. \& Tosques, M., Evolution equations with lack of convexity, Nonlinear Analysis 9 (1985), 1401-1443. MR820649 (87h:35147)

[22] De Giorgi, E., Marino, A. \& Tosques, M., Problems of evolution in metric spaces and maximal decreasing curve, Atti Accad. Naz. Lincei Rend. Cl. Sci. Fis. Mat. Natur. 68 (1980), 180-187. MR636814 (83m:49052)

[23] Dontchev, A.L., Lewis, A.S. \& Rockafellar, R.T., The radius of metric regularity, Trans. Amer. Math. Soc. 335 (2002), 493-517. MR1932710 (2003i:49026)

[24] Dontchev, A. L., Quincampoix, M. \& Zlateva, N., Aubin criterion for metric regularity, J. Convex Anal. 13 (2006), 281-297. MR2252233 (2007d:49026)

[25] van den Dries, L. \& Miller, C., Geometric categories and o-minimal structures, Duke Math. J. 84 (1996), 497-540. MR.1404337 (97i:32008)

[26] Evans, L. C. \& Spruck, J., Motion of level sets by mean curvature. III, J. Geom. Anal. 2 (1992) 121-150. MR1151756 (93d:58044)

[27] Fenchel, W., Convex Cones, Sets and Functions, Mimeographed lecture note, Princeton University, 1951.

[28] Forti, M., Nistri, P. \& Quincampoix, M., Convergence of Neural Networks for Programming Problems via a Nonsmooth Łojasiewicz Inequality, IEEE Trans. on Neural Networks, 17 (2006), 1471-1486.

[29] Gage, M. \& Hamilton, R. S., The heat equation shrinking convex plane curves, J. Differential Geom. 23 (1986) 69-96. MR840401 (87m:53003)

[30] Haraux, A., A hyperbolic variant of Simon's convergence theorem. Evolution equations and their applications in physical and life sciences (Bad Herrenalb, 1998), Lecture Notes in Pure and Appl. Math. 215 (2001), 255-264, Marcel Dekker, New York. MR.1818007(2002a:35147)

[31] Huang, S.-Z. Gradient inequalities. With applications to asymptotic behavior and stability of gradient-like systems, Mathematical Surveys and Monographs, 126, American Mathematical Society, Providence, RI, 2006. MR2226672 (2007b:35035)

[32] Ioffe, A. Metric regularity and Subdifferential Calculus, Russian Math. Surveys 55 (2000), 501-558 MR1777352(2001j:90002)

[33] Ioffe, A., "Towards metric theory of metric regularity", in: Approximation, optimization and mathematical economics, (Pointe-à-Pitre, 1999), 165-176, Physica, Heidelberg, 2001. MR 1842886 (2002f:49034)

[34] Ioffe, A., On regularity estimates for mappings between embedded manifolds, Control Cybernet. 36 (2007), 659-668. MR2376046 (2009c:49042)

[35] Jendoubi, M., A simple unified approach to some convergence theorems of L. Simon, J. Funct. Anal. 153 (1998), 187-202. MR 1609269 (99c:35101)

[36] Kannai, Y., Concavifiability and constructions of concave utility functions, J. Math. Econom. 4 (1977), 1-56. MR0459523(56:17715)

[37] Kurdyka, K., On gradients of functions definable in o-minimal structures, Ann. Inst. Fourier 48 (1998), 769-783. MR1644089 (2000b:03139)

[38] Kurdyka, K. \& Parusinski, A., $w_{f}$-stratification of subanalytic functions and the Lojasiewicz inequality, C. R. Acad. Sci. Paris Sér. I Math. 318 (1994), 129-133. MR.1260324 (95d:32012)

[39] Lageman, C., Convergence of gradient-like dynamical systems and optimization algorithms, Ph.D. Thesis, University of Würzburg, (2007), 205 pp. 
[40] Lee, J. M., Introduction to smooth manifolds, Graduate Texts in Mathematics, 218. SpringerVerlag, New York, 2003. xviii+628 pp. MR1930091 (2003k:58001)

[41] Lemaire, B., An Asymptotical Variational Principle Associated with the Steepest Descent Method for a Convex Function, J. Convex Anal. 3 (1996), 63-70. MR1422752 (97m:90096)

[42] Łojasiewicz, S., "Une propriété topologique des sous-ensembles analytiques réels.", in: Les Équations aux Dérivées Partielles, pp. 87-89, Éditions du centre National de la Recherche Scientifique, Paris 1963. MR0160856 (28:4066)

[43] Łojasiewicz, S., Ensembles semi-analytiques, preprint 112 pp. (IHES, 1965). Available at http://perso.univ-rennes1.fr/michel.coste/Lojasiewicz.pdf

[44] Marcellin, S. \& Thibault, L., Evolution problems associated with primal lower nice functions, J. Convex Anal. 13 (2006), 385-421. MR2252239(2007e:49029)

[45] Mordukhovich, B., Complete characterization of openness, metric regularity and Lipschitzian properties of multifunctions. Trans. Amer. Math. Soc. 340 (1993), 1-35. MR 1156300 (94a:49011)

[46] Mordukhovich, B., Variational analysis and generalized differentiation. I. Basic theory, Grundlehren der Mathematischen Wissenschaften [Fundamental Principles of Mathematical Sciences], 330. Springer-Verlag, Berlin, 2006. xxii+579 pp. MR2191744 (2007b:49003a)

[47] Nesterov, Y. \& Polyak, B. T., Cubic regularization of Newton method and its global performance. Math. Program. 108 (2006), no. 1, Ser. A, 177-205. MR2229459 (2007f:90153)

[48] Nistri, P. \& Quincampoix, M. On the properties of solutions to a differential inclusion associated with a nonsmooth constrained optimization problem, Proceedings of the 44th IEEE Conference on Decision and Control and the European Control Conference 2005, Seville, Spain, December 12-15, 2005.

[49] Penot, J.-P., Metric regularity, openness and Lipschitzian behaviour of multifunctions. Nonlinear Analysis, 13 (1989), 629-643. MR998509(90h:54024)

[50] Peypouquet, J., Analyse asymptotique de systèmes d'évolution et applications en optimisation, 116 pp. (Université Paris 6 \& Universidad de Chile, 2007).

[51] Simon, L., Asymptotics for a class of non-linear evolution equations, with applications to geometric problems, Ann. Math. 118 (1983), 525-571. MR727703(85b:58121)

[52] Torralba, D., Convergence épigraphique et changements d'échelle en analyse variationnelle et optimisation, 160 pp., Ph.D. Thesis (Université de Montpellier 2, 1996).

[53] Rockafellar, R.T. \& Wets, R., Variational Analysis, Grundlehren der Mathematischen Wissenschaften, Vol. 317, Springer, 1998. MR1491362 (98m:49001)

[54] Zhu, X. Lectures on mean curvature flows, AMS/IP Studies in Advanced Mathematics 32, American Mathematical Society, 2002. MR1931534 (2004f:53084)

UPMC Université Paris 06 - Équipe Combinatoire et Optimisation (UMR 7090), Case 189, Université Pierre et Marie Curie, 4 Place Jussieu, F-75252 Paris Cedex 05, France

E-mail address: bolte@math.jussieu.fr

Departament de Matemàtiques, C1/308, Universitat Autònoma de Barcelona, E08193 Bellaterra (Cerdanyola del Vallès), Spain

E-mail address: arisd@mat.uab.es

Laboratoire de Mathématiques et Physique Théorique (CNRS UMR 6083), Fédération Denis Poisson, Faculté des Sciences et Techniques, Université François Rabelais, Parc De Grandmont, F-37200 Tours, France

E-mail address: ley@lmpt.univ-tours.fr

Current address: IRMAR (CNRS UMR 6625) INSA de Rennes, 20 avenue des buttes de Coesmes, F-35708 Rennes Cedex 7, France

E-mail address: olivier.ley@univ-rennes1.fr

Université Paris-Est, Laboratoire D’Analyse et Mathématiques Appliquées, UMR 8050, UfR des Sciences et Technologie, Département de Mathématiques, 61 avenue du Général de Gaulle 94010 Créteil Cedex, France

E-mail address: laurent.mazet@univ-paris12.fr 